Supporting Information

\title{
Tris(pentafluorophenyl)borane-Catalyzed Cyclopropanation of Styrenes with Arydiazoacetates
}

\author{
Joseph P. Mancinelli and Sidney M. Wilkerson-Hill ${ }^{*}$ \\ ${ }^{\dagger}$ Department of Chemistry, The University of North Carolina at Chapel Hill, Chapel Hill, North \\ Carolina 27599-3290, United States of America \\ *smwhill@email.unc.edu

\section{TABLE OF CONTENTS}

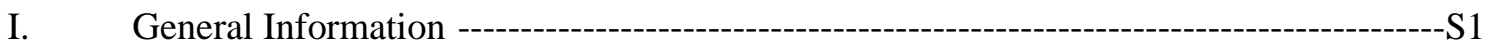

II. General Procedures for the Synthesis of Starting Materials ----o---S2

III. Optimization Table------

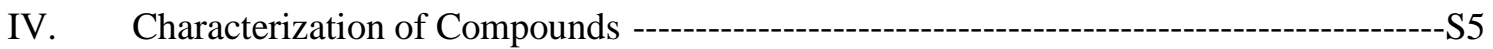

V. Control experiment for cyclopropane stability in the presence of $\mathrm{B}\left(\mathrm{C}_{6} \mathrm{~F}_{5}\right)_{3}{ }^{--------------S 23}$

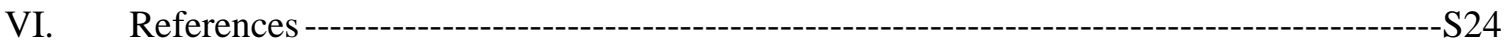

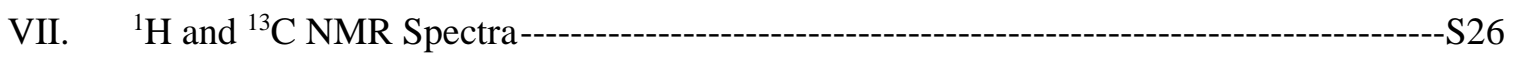




\section{General Information}

Reaction setup and reagent synthesis: All reactions were carried out under a nitrogen atmosphere in flame-dried glassware using standard Schlenk techniques unless otherwise stated. Reactions that were extremely moisture and oxygen sensitive reactions were performed within a Vigor glove box (box atmospheric pressure ranged from 3-5 atm, moisture and oxygen ranged from $0.10-0.20 \mathrm{ppm}$ and 0.01 and $0.02 \mathrm{ppm}$ respectively). Tris(pentafluorophenyl)borane $\left(\mathrm{B}\left(\mathrm{C}_{6} \mathrm{~F}_{5}\right)_{3}\right)$ was purchased from TCI Chemicals and used without further purification. All other reagents were purchased from commercial sources and were used without purification unless otherwise noted. Methyl 2-(4-bromophenyl)-2-diazoacetate (1a), Methyl 2-phenyl-2-diazoacetate (1b), methyl 2-(4-methylphenyl)-2-diazoacetate (1c), methyl 2-(4-chlorophenyl)-2-diazoacetate (1d), methyl 2-(4-methoxyphenyl)-2-diazoacetate (1e), methyl 2-(4-fluorophenyl)-2-diazo acetate (1f) methyl 2-(4-tert-butylphenyl)-2-diazoacetate (1g), ethyl 2-(4-bromophenyl)-2-diazoacetate (1h), isopropyl 2-(4-bromophenyl)-2-diazoacetate (1i), 2,2,2-trichloroethyl (2-(4-bromophenyl)-

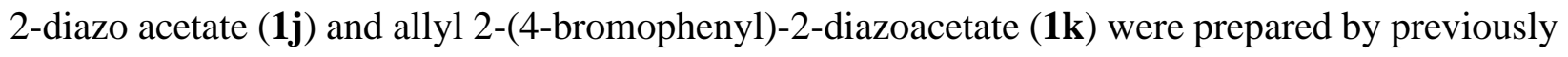
published methods. ${ }^{1-6}$ Styrenes 4-vinylbiphenyl (2e), 3-vinylanisole (2f), 3-chlorostyrene (2g), 2chlorostyrene (2h), 2-vinylnaphthalene (2j), 1,2-dihydronaphthalene (2k) and tosylvinylindole (2m) were prepared by previously published methods $s^{7,8,9}$ and fully matched previous

characterization. ${ }^{8-14}$ 4-acetamidobenzenesulfonyl azide was synthesized by known procedures. ${ }^{15}$ CAUTION: Diazo compounds are potentially shock sensitive and should be handled with care. ${ }^{16}$

Solvents: Toluene, THF, dichloromethane, diethyl ether, acetonitrile and triethylamine were purified using a GlassContour solvent purification system purchased from Pure Process Technologies. Other solvents (hexanes and pentanes) were purified by refluxing over activated 3 $\AA$ molecular sieves (beads) for 1 hour and stored as such under a balloon filled with argon. 
Anhydrous 1,2-dichloroethane, 1,4-dioxane, and dimethyl formamide were purchased from Sigma-Aldrich and were used without purification.

Chromatography: Analytical thin layer chromatography (TLC) was performed on SiliaPlate glass backed silica gel plates ( $250 \mu \mathrm{m}$ thickness, $60 \AA$, F-254 indicator) purchased from Silicycle and visualized using ultraviolet light (254 and $200 \mathrm{~nm}$ ) and stained with $\mathrm{KMNO}_{4}$, $p$-anisaldehyde, cerium molybdate, ceric ammonium nitrate, vanillin, or ninhydrin made following reported recipes. ${ }^{17}$ Flash column chromatography was performed with SiliaFlash silica gel $60 \AA$ A, 230-400 mesh $(40-63 \mu \mathrm{m})$ purchased from Silicycle according to the literature procedure. ${ }^{18}$

Melting points: All melting points were measured on powdered solids using a Mel-Temp II capillary melting point device and are uncorrected unless otherwise stated.

Nuclear Magnetic Resonance Characterization: ${ }^{1} \mathrm{H}$ NMR and ${ }^{13} \mathrm{C}$ NMR spectra were recorded on a Bruker AV Neo-400 (equipped with a Prodigy cryoprobe), Bruker AV III NanoBay-400, Bruker AV III-500, Bruker AV Neo-600 (equipped with a CryoQNP probe) or Bruker AV III-600 MHz spectrometer $\left(100,125\right.$, or $150 \mathrm{MHz}$ for ${ }^{13} \mathrm{C}$ NMR). All spectra were recorded in deuterated chloroform $\left(\mathrm{CDCl}_{3}\right)$ with residual chloroform $\left(\delta=7.26 \mathrm{ppm}\right.$ for ${ }^{1} \mathrm{H}$ NMR and 77.16 for $\left.{ }^{13} \mathrm{C} \mathrm{NMR}\right)$ as the internal standard and are reported in parts per million ( $\mathrm{ppm})$. Abbreviations for the signal couplings are as follows: s, singlet; $\mathrm{d}$, doublet; $\mathrm{t}$, triplet; q, quartet; quin, quintet; sex, sextet; sept; septet; and m, multiplet. Coupling constants were taken from the spectra directly and are uncorrected. Structural and stereochemical assignments were also made based on analysis of ${ }^{13} \mathrm{C}$ APT experiment, 2-D ${ }^{1} \mathrm{H}^{-1} \mathrm{H}$ COSY and ${ }^{1} \mathrm{H}^{-1} \mathrm{H}$ NOESY, ${ }^{1} \mathrm{H}^{-13} \mathrm{C}$ HSQC and ${ }^{1} \mathrm{H}^{-13} \mathrm{C}$ HMBC experiments. All diastereomeric ratios were determined by ${ }^{1} \mathrm{H}$ NMR analysis of an aliquot from the crude reaction mixture. Yields refer to isolated yields of compounds estimated to be $\geq 95 \%$ 
pure as determined by ${ }^{1} \mathrm{H}$ NMR analysis unless otherwise noted. Analytically pure samples for characterization were obtained by preparative TLC.

Infrared Spectroscopy Characterization: Infrared (IR) spectra were collected on a Thermo Scientific Nicolet iS 5 FT-IR. Compounds were analyzed as thin films or as solids on the diamond surface. Some samples were analyzed on a Jasco 460 Plus FT-IR. Compounds were analyzed as thin films on $\mathrm{KBr}$. Spectra are reported in frequency of absorption in $\mathrm{cm}^{-1}$. Only selected references are reported.

Mass spectrometry: High-resolution mass spectra (HRMS) determinations were performed by the mass spectrometry core at the University of North Carolina at Chapel Hill. Samples were analyzed with a Q Exactive HF-X (ThermoFisher, Bremen, Germany) mass spectrometer for APCI and ESI.

\section{General procedures for synthesis of starting materials}

\section{A. General procedure for the synthesis of diazo compounds:}

An oven-dried $250 \mathrm{~mL}$ round bottomed flask equipped with a stir bar was charged with aryl acetic acid (42.0 mmol, 1.00 equiv), alcohol (40.0 mL) and concentrated sulfuric acid solution (3.72 $\mathrm{mL}, 69.8 \mathrm{mmol}, 3.00$ equiv). The reaction mixture was heated to reflux overnight. The reaction mixture was then cooled to room temperature and concentrated under reduced pressure. The resulting residue was partitioned between water $(30 \mathrm{~mL})$ and diethyl ether $(30 \mathrm{~mL})$ and the aqueous phase was extracted with diethyl ether $(3 \times 30 \mathrm{~mL})$. The organic layers were combined, washed with a saturated aqueous solution of $\mathrm{NaHCO}_{3}(2 \times 20 \mathrm{~mL})$ dried over $\mathrm{MgSO}_{4}$, filtered and concentrated under reduced pressure to afford the aryl acetic acid ester as a clear oil which was used without further purification.

Adapting from a procedure by Keipour and coworkers, ${ }^{1}$ the aryl acetic acid ester (13.1 mmol, 1.00 equiv) and 4-acetamidobenzenesulfonyl azide (19.7 mmol, 1.50 equiv) were added to an oven-dried $100-\mathrm{mL}$ round-bottom flask containing a stir bar and dissolved in dry acetonitrile (40 mL). The reaction was cooled to $0{ }^{\circ} \mathrm{C}$ and 1,8-diazabicyclo[5.4.0]undec-7-ene (DBU) (2.35 
$\mathrm{mL}, 15.7 \mathrm{mmol}, 1.20$ equiv) was added dropwise. The reaction was warmed to room temperature and stirred overnight. Saturated $\mathrm{NH}_{4} \mathrm{Cl}(\mathrm{aq})(40 \mathrm{~mL})$ was added to the flask. The aqueous phase and organic phases were separated, and the aqueous phase was extracted with diethyl ether $(3 \times$ $30 \mathrm{~mL})$. The organic phases were combined and washed with brine $(2 \times 30 \mathrm{~mL})$, dried over $\mathrm{MgSO}_{4}$, and concentrated under reduced pressure. The crude reaction mixture was then purified by column chromatography to afford the product.

\section{B. General procedure for the synthesis of styrene derivatives:}

Styrene derivatives $\mathbf{2 e -} \mathbf{2 h}$, and $\mathbf{2} \mathbf{j}$ are commercially available but were synthesized using a procedure adapted from Meek and coworkers. ${ }^{7}$ An oven dried $100 \mathrm{~mL}$ round-bottom flask was equipped with a stir bar and methyltriphenylphosphonium iodide (4.05 g, $10.0 \mathrm{mmol})$ was added. The flask was evacuated and refilled with nitrogen $(\times 3)$. To the solids was added dry THF $(30$ $\mathrm{mL}, 0.33 \mathrm{M})$. Potassium tert-butoxide $(1.12 \mathrm{~g}, 10.0 \mathrm{mmol})$ was added to the flask and the mixture was stirred for 2 hours. The benzaldehyde derivative $(9.93 \mathrm{mmol})$ was added by syringe and the mixture was stirred for 18 hours. Saturated $\mathrm{NH}_{4} \mathrm{Cl}(\mathrm{aq})(15 \mathrm{~mL})$ was added to the flask. The aqueous layer was then extracted with dichloromethane $(2 \times 15 \mathrm{~mL})$ and the combined organic layers were washed with brine $(3 \times 15 \mathrm{~mL})$, dried over magnesium sulfate, and concentrated under reduced pressure. The styrene derivatives were purified via flash chromatography and were used immediately but can be stored under nitrogen atmosphere in a freezer for several days. All data for styrene derivatives matched previously reported values. ${ }^{8-12}$

\section{General procedure for $B\left(C_{6} F_{5}\right)_{3}$-catalyzed cyclopropanation reactions:}

In a nitrogen-filled glove box, tris(pentafluorophenyl)borane $(15.0 \mu \mathrm{mol})$ and aryldiazoacetate $(300 \mu \mathrm{mol})$ were weighed into a $10-\mathrm{mL}$ reaction tube with a stir bar. The tube was capped with a Teflon-coated septa cap and removed from the glovebox. To this mixture was added dry 1,2-dichloroethane $(1.5 \mathrm{~mL}, 0.10 \mathrm{M})$. The solution was then stirred at room temperature for 5 minutes. Excess alkene was then passed through a plug of silica-gel into a separate 4-dram vial and capped with a Teflon-coated septa cap. The vial was evacuated and refilled with nitrogen once and the alkene $(150 \mu \mathrm{mol})$ was then added to the solution containing $\mathrm{B}\left(\mathrm{C}_{6} \mathrm{~F}_{5}\right)_{3}$ and aryldiazoacetate, heated to $50{ }^{\circ} \mathrm{C}$, and stirred for 16 hours unless otherwise stated. The reaction was then cooled to room temperature and concentrated under reduced pressure. The residue was 
dry loaded onto silica gel and purified using column chromatography to isolate the cyclopropane product.

\section{General procedure for optimization of $\mathrm{B}\left(\mathrm{C}_{6} \mathrm{~F}_{5}\right)_{3}$-catalyzed cyclopropanation reactions:}

Following general procedure $\mathrm{C}$, the amounts of tris(pentafluorophenyl)borane, styrene, diazo, temperature, time and catalysts were changed as indicated below. After $16 \mathrm{~h}$, the reaction mixture was concentrated and internal standard (150 $\mu \mathrm{mol}$ hexamethyldisiloxane) was added and the mixture was analyzed by ${ }^{1} \mathrm{H}$ NMR.

Table S1: Full optimization of $\mathrm{B}\left(\mathrm{C}_{6} \mathrm{~F}_{5}\right)_{3}$-catalyzed cyclopropanation reaction.

\begin{tabular}{|c|c|c|c|c|c|c|c|}
\hline Entry & catalyst & loading (mol\%) & temp. $\left({ }^{\circ} \mathrm{C}\right)$ & solvent & 1a (equiv) & styrene (equiv) & yield(\%) 3 \\
\hline 1 & $\mathrm{~B}\left(\mathrm{C}_{6} \mathrm{~F}_{5}\right)_{3}$ & 5 & 50 & $\mathrm{PhMe}$ & 1 & 5 & 27 \\
\hline 2 & $\mathrm{~B}\left(\mathrm{C}_{6} \mathrm{~F}_{5}\right)_{3}$ & 10 & 50 & $\mathrm{PhMe}$ & 1 & 5 & 33 \\
\hline 3 & $\mathrm{~B}\left(\mathrm{C}_{6} \mathrm{~F}_{5}\right)_{3}$ & 15 & 50 & $\mathrm{PhMe}$ & 1 & 5 & 21 \\
\hline 4 & $\mathrm{~B}\left(\mathrm{C}_{6} \mathrm{~F}_{5}\right)_{3}$ & 75 & 50 & $\mathrm{PhMe}$ & 1 & 5 & $<5$ \\
\hline 5 & $\mathrm{~B}\left(\mathrm{C}_{6} \mathrm{~F}_{5}\right)_{3}$ & 5 & 50 & $\mathrm{Et}_{2} \mathrm{O}$ & 1 & 5 & 37 \\
\hline 6 & $\mathrm{~B}\left(\mathrm{C}_{6} \mathrm{~F}_{5}\right)_{3}$ & 5 & 50 & THF & 1 & 5 & $<5$ \\
\hline 7 & $\mathrm{~B}\left(\mathrm{C}_{6} \mathrm{~F}_{5}\right)_{3}$ & 5 & 50 & $\mathrm{MeCN}$ & 1 & 5 & $<5$ \\
\hline 8 & $\mathrm{~B}\left(\mathrm{C}_{6} \mathrm{~F}_{5}\right)_{3}$ & 5 & 50 & $\mathrm{CH}_{2} \mathrm{Cl}_{2}$ & 1 & 5 & 16 \\
\hline 9 & $\mathrm{~B}\left(\mathrm{C}_{6} \mathrm{~F}_{5}\right)_{3}$ & 5 & 50 & $\mathrm{PhH}$ & 1 & 5 & 57 \\
\hline 10 & $\mathrm{~B}\left(\mathrm{C}_{6} \mathrm{~F}_{5}\right)_{3}$ & 5 & 25 & $\mathrm{PhH}$ & 1 & 5 & 12 \\
\hline 11 & $\mathrm{~B}\left(\mathrm{C}_{6} \mathrm{~F}_{5}\right)_{3}$ & 5 & 0 & $\mathrm{PhH}$ & 1 & 5 & 10 \\
\hline 12 & $\mathrm{~B}\left(\mathrm{C}_{6} \mathrm{~F}_{5}\right)_{3}$ & 5 & -78 & $\mathrm{PhH}$ & 1 & 5 & 11 \\
\hline 13 & $\mathrm{~B}\left(\mathrm{C}_{6} \mathrm{~F}_{5}\right)_{3}$ & 5 & 50 & $\mathrm{PhH}$ & 1 & 4 & 10 \\
\hline 14 & $\mathrm{~B}\left(\mathrm{C}_{6} \mathrm{~F}_{5}\right)_{3}$ & 5 & 50 & $\mathrm{PhH}$ & 1 & 3 & 36 \\
\hline 15 & $\mathrm{~B}\left(\mathrm{C}_{6} \mathrm{~F}_{5}\right)_{3}$ & 5 & 50 & $\mathrm{PhH}$ & 1 & 2 & 14 \\
\hline 16 & $\mathrm{~B}\left(\mathrm{C}_{6} \mathrm{~F}_{5}\right)_{3}$ & 5 & 50 & $\mathrm{PhH}$ & 1 & 1 & 66 \\
\hline 17 & $\mathrm{~B}\left(\mathrm{C}_{6} \mathrm{~F}_{5}\right)_{3}$ & 5 & 50 & 1,2-DCE & 1 & 1 & 70 \\
\hline 18 & $\mathrm{~B}\left(\mathrm{C}_{6} \mathrm{~F}_{5}\right)_{3}$ & 5 & 50 & 1,2-DCE & 1 & 5 & $63^{a}$ \\
\hline 19 & $\mathrm{~B}\left(\mathrm{C}_{6} \mathrm{~F}_{5}\right)_{3}$ & 10 & 50 & 1,2-DCE & 2 & 1 & $83^{a, b}$ \\
\hline 20 & $\mathrm{BPh}_{3}$ & 10 & 50 & 1,2-DCE & 2 & 1 & $<5$ \\
\hline 21 & $\mathrm{BF}_{3} \cdot \mathrm{OEt}_{2}$ & 10 & -78 to 25 & $\mathrm{CH}_{2} \mathrm{Cl}_{2}$ & 2 & 1 & $21^{\mathrm{a}}$ \\
\hline 22 & $\mathrm{SnCl}_{4}$ & 10 & -78 to 25 & $\mathrm{CH}_{2} \mathrm{Cl}_{2}$ & 2 & 1 & $21^{a}$ \\
\hline 23 & $\mathrm{TiCl}_{4}$ & 10 & -78 to 25 & $\mathrm{CH}_{2} \mathrm{Cl}_{2}$ & 2 & 1 & decomp. \\
\hline 24 & no catalysts & - & 50 & 1,2-DCE & 2 & 1 & S.M. \\
\hline 25 & $\mathrm{~B}\left(\mathrm{C}_{6} \mathrm{~F}_{5}\right)_{3}$ & 10 & 50 & 1,2-DCE & 2 & 1 & $87^{c}$ \\
\hline
\end{tabular}

Standard conditions: $0.10 \mathrm{M}, 16 \mathrm{~h}$, and $150 \mu \mathrm{mol}$ hexamethyldisiloxane as an internal standard.

${ }^{\text {a}}$ Yields are based on ${ }^{1} \mathrm{H}$ NMR analysis of the crude reaction mixture using $150 \mu \mathrm{mol}$ mesitylene as an internal standard. ${ }^{\mathrm{b}} 150 \mu \mathrm{mol}$ styrene $\mathbf{2 a}, 300 \mu \mathrm{mol}$ ( 2 equiv) diazo 1a. ${ }^{\mathrm{c}}$ Reaction performed in the dark. 


\section{Characterization data for the cyclopropane products}

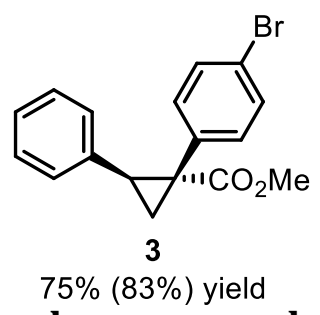

Methyl 1-(4-bromophenyl)-2-phenylcyclopropanecarboxylate Following general procedure C, 3 was obtained as an off-white solid ( ${ }^{1}$ H NMR yield: $83 \%$, isolated yield: $75 \%, 37.2 \mathrm{mg}$.) When run using $1.00 \mathrm{mmol}$ styrene $(232 \mathrm{mg}, 0.70 \mathrm{mmol}) 70 \%$ isolated yield of 3a was obtained. Rf: 0.37 (pentane/ethyl acetate 100:1).

${ }^{1} \mathbf{H}$ NMR $\left(600 \mathrm{MHz} \mathrm{CDCl}_{3}\right) \delta 7.25(\mathrm{~d}, J=8.5 \mathrm{~Hz}, 2 \mathrm{H}), 7.11-7.07$ (m, $\left.3 \mathrm{H}\right), 6.89$ (d, J = 8.5 Hz, $2 \mathrm{H}), 6.79-6.75(\mathrm{~m}, 2 \mathrm{H}), 3.66$ (s, $3 \mathrm{H}), 3.11(\mathrm{dd}, J=9.3,7.3 \mathrm{~Hz}, 1 \mathrm{H}), 2.14$ (dd, $J=9.3,5.0 \mathrm{~Hz}$, $1 \mathrm{H}), 1.84(\mathrm{dd}, J=7.4,5.0 \mathrm{~Hz}, 1 \mathrm{H})$.

${ }^{13} \mathrm{C}$ NMR (151 MHz, $\left.\mathrm{CDCl}_{3}\right) \delta 173.9$ 136.0, 134.1, 133.7, 131.0, 128.2, 128.1, 126.7, 121.3, $52.8,36.9,33.3,20.5$. All data matches data previously reported in the literature. ${ }^{19}$

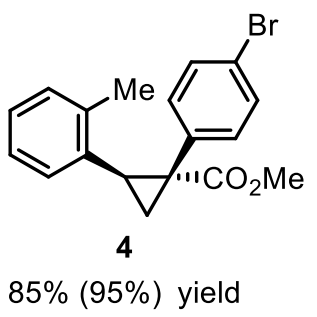

Methyl 1-(4-bromophenyl)-2-(2-methylphenyl) cyclopropanecarboxylate

Reaction conducted on $69 \mu \mathrm{mol}$ scale following general procedure C. 4 was obtained as an offwhite solid ( ${ }^{1}$ H NMR yield: 95\%, isolated yield: 85\%, $20.3 \mathrm{mg}$ ).

Rf: 0.21 (hexanes/ethyl acetate 19:1).

m.p. $147-149{ }^{\circ} \mathrm{C}$.

${ }^{1} \mathrm{H}$ NMR $\left(600 \mathrm{MHz}, \mathrm{CDCl}_{3}\right) \delta 7.19(\mathrm{~d}, J=8.5 \mathrm{~Hz}, 2 \mathrm{H}), 7.13-7.10(\mathrm{~m}, 1 \mathrm{H}), 7.03-6.99$ (m, $\left.1 \mathrm{H}\right)$, 6.86-6.81 (m, 3 H), 6.40 (d, J=7.7 Hz, $1 \mathrm{H}), 3.69$ (s, $3 \mathrm{H}), 3.14(\mathrm{dd}, J=9.1,7.9 \mathrm{~Hz}, 1 \mathrm{H}), 2.46$ (s, $3 \mathrm{H}), 2.07$ (dd, $J=9.2,4.9 \mathrm{~Hz}, 1 \mathrm{H}), 2.00(\mathrm{dd}, J=7.6,4.9 \mathrm{~Hz}, 1 \mathrm{H})$.

${ }^{13} \mathrm{C}$ NMR $\left(151 \mathrm{MHz}, \mathrm{CDCl}_{3}\right) \delta 174.1,137.9,134.3,133.9,130.0,131.0,129.9,126.9,125.8$, 125.7, 121.2, 52.9, 35.9, 31.3, 20.2, 19.0.

IR (thin film, KBr plate) 3055, 2986, 1722, 1371, 1265, 896, 740, $705 \mathrm{~cm}^{-1}$.

HRMS-APCI $(m / z)$ calc'd for $\mathrm{C}_{18} \mathrm{H}_{18} \mathrm{O}_{2} \mathrm{Br}[\mathrm{M}+\mathrm{H}]^{+}: 345.0485$, found 345.0487. 


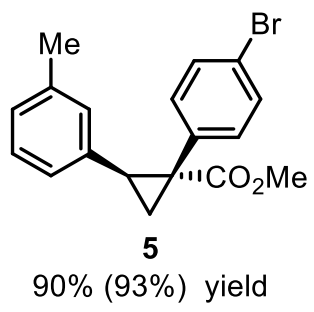

\section{Methyl 1-(4-bromophenyl)-2-(3-methylphenyl) cyclopropanecarboxylate}

Following general procedure C, 5 was obtained as an off-white solid ( ${ }^{1} \mathbf{H}$ NMR yield: $93 \%$, isolated yield: $90 \%, 46.8 \mathrm{mg}$ ).

Rf: 0.24 (pentane/ethyl acetate 100:1).

m.p. $98-102{ }^{\circ} \mathrm{C}$.

${ }^{1} \mathbf{H}$ NMR $\left(400 \mathrm{MHz}, \mathrm{CDCl}_{3}\right) \delta 7.25(\mathrm{~d}, J=8.4 \mathrm{~Hz}, 2 \mathrm{H}), 6.98-6.94(\mathrm{~m}, 1 \mathrm{H}), 6.92-6.87$ (m, $\left.3 \mathrm{H}\right)$, 6.65 (m, $1 \mathrm{H}), 6.49$ (m, $2 \mathrm{H}), 3.66$ (s, $3 \mathrm{H}), 3.06$ (dd, $J=9.3,7.3 \mathrm{~Hz}, 1 \mathrm{H}), 2.18$ (s, $3 \mathrm{H}), 2.12$ (dd, $J=9.3,4.9 \mathrm{~Hz}, 1 \mathrm{H}), 1.82(\mathrm{dd}, J=7.3,5.0 \mathrm{~Hz}, 1 \mathrm{H})$.

${ }^{13} \mathrm{C}$ NMR $\left(151 \mathrm{MHz}, \mathrm{CDCl}_{3}\right) \delta 174.0,137.6,135.9,134.2,133.7,131.0,129.3,127.9,127.5$, $125.0,121.3,52.8,36.8,33.3,21.4,20.5$.

IR (thin film, KBr plate) 3445, 2950, 1719, 1273, 1073, 1011, 788, $719 \mathrm{~cm}^{-1}$.

HRMS $(m / z)$ calc'd for $\mathrm{C}_{18} \mathrm{H}_{18} \mathrm{O}_{2} \mathrm{Br}[\mathrm{M}+\mathrm{H}]^{+}:$345.0485, found 345.0486.

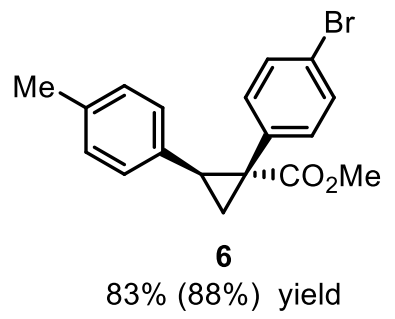

Methyl 1-(4-bromophenyl)-2-(4-methylphenyl) cyclopropanecarboxylate

Following general procedure C, 3d was obtained as an off-white solid ( ${ }^{1} \mathbf{H}$ NMR yield: $88 \%$, isolated yield: $83 \%, 43.0 \mathrm{mg}$ ).

Rf: 0.29 (pentane/ethyl acetate 100:1).

m.p. $130-132{ }^{\circ} \mathrm{C}$.

${ }^{1} \mathrm{H}$ NMR $\left(500 \mathrm{MHz}, \mathrm{CDCl}_{3}\right) \delta 7.26(\mathrm{~d}, J=8.2 \mathrm{~Hz}, 2 \mathrm{H}), 6.91-6.87(\mathrm{~m}, 4 \mathrm{H}), 6.65(\mathrm{~d}, J=8.2 \mathrm{~Hz}$, $2 \mathrm{H}), 3.65$ (s, $3 \mathrm{H}), 3.07$ (dd, $J=9.3,7.3 \mathrm{~Hz}, 1 \mathrm{H}), 2.23$ (s, $3 \mathrm{H}), 2.12(\mathrm{dd}, J=9.3,4.9 \mathrm{~Hz}, 1 \mathrm{H})$, $1.79(\mathrm{dd}, J=7.3,4.9 \mathrm{~Hz}, 1 \mathrm{H})$.

${ }^{13} \mathrm{C}$ NMR $\left(151 \mathrm{MHz}, \mathrm{CDCl}_{3}\right) \delta 174.0,136.3,134.3,133.7,132.8,131.0,128.8,128.0,121.3,52.8$, $36.7,33.1,21.1,20.5$. 
IR (ATR) 2955, 2923, 1718, 1255, 1159, 1011, 824, $727 \mathrm{~cm}^{-1}$.

HRMS-APCI $(\mathrm{m} / \mathrm{z})$ calc'd for $\mathrm{C}_{18} \mathrm{H}_{18} \mathrm{O}_{2} \mathrm{Br}[\mathrm{M}+\mathrm{H}]^{+}: 345.0485$, found 345.0485.
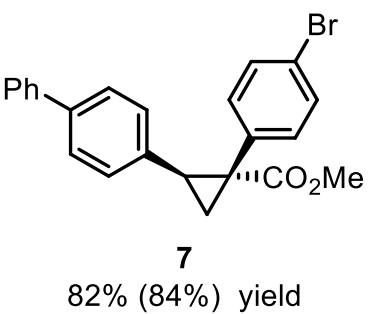

Methyl 1-(4-bromophenyl)-2-(biphenyl) cyclopropanecarboxylate Following general procedure C, 7 was obtained as an off-white solid ( ${ }^{1}$ H NMR yield: $84 \%$, isolated yield: 82\%, 50.3 $\mathrm{mg})$.

Rf: 0.34 (petroleum ether/ether 18:1).

m.p. $168-170{ }^{\circ} \mathrm{C}$.

${ }^{1} \mathbf{H}$ NMR $\left(600 \mathrm{MHz}, \mathrm{CDCl}_{3}\right) \delta$ 7.54-7.50 (m, $\left.2 \mathrm{H}\right), 7.41-7.37$ (m, $\left.2 \mathrm{H}\right), 7.35-7.32$ (m, $\left.2 \mathrm{H}\right), 7.32-$ $7.29(\mathrm{~m}, 1 \mathrm{H}) 7.27$ (d, $J=8.4 \mathrm{~Hz}, 2 \mathrm{H}) 6.93(\mathrm{~d}, J=8.4 \mathrm{~Hz}, 2 \mathrm{H}), 6.85-6.81(\mathrm{~m}, 2 \mathrm{H}), 3.67(\mathrm{~s}, 3 \mathrm{H})$, $3.14(\mathrm{dd}, J=9.3,7.3 \mathrm{~Hz}, 1 \mathrm{H}), 2.18(\mathrm{dd}, J=9.3,5.0 \mathrm{~Hz}, 1 \mathrm{H}), 1.86(\mathrm{dd}, J=7.2,4.9 \mathrm{~Hz}, 1 \mathrm{H})$.

${ }^{13}$ C NMR $\left(151 \mathrm{MHz}, \mathrm{CDCl}_{3}\right) \delta 173.9,140.6,139.4,135.2,134.0,133.7,131.1,128.8,128.5$, 127.3, 127.0, 126.6, 121.4.

IR (ATR) 3028, 2924, 1718, 1255, 1162, 1011, 770, $740 \mathrm{~cm}^{-1}$.

HRMS-APCI $(\mathrm{m} / \mathrm{z})$ calc'd for $\mathrm{C}_{23} \mathrm{H}_{20} \mathrm{O}_{2} \mathrm{Br}[\mathrm{M}+\mathrm{H}]^{+}:$407.0641, found 407.0644.

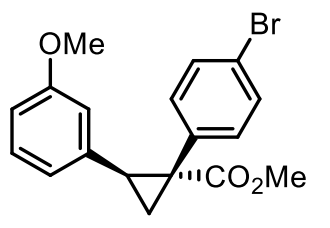

8

$79 \%(82 \%)$ yield

\section{Methyl 1-(4-bromophenyl)-2-(3-methoxyphenyl) cyclopropanecarboxylate}

Following general procedure C, 8 was obtained as a low melting solid ( ${ }^{1} \mathbf{H}$ NMR yield: $82 \%$, isolated yield 79\%, $42.6 \mathrm{mg}$ ).

Rf: 0.24 (pentane/ethyl acetate 30:1).

${ }^{1} \mathbf{H}$ NMR $\left(600 \mathrm{MHz}, \mathrm{CDCl}_{3}\right) \delta 7.26(\mathrm{~d}, J=8.4 \mathrm{~Hz}, 2 \mathrm{H}), 7.00(\mathrm{~m}, 1 \mathrm{H}), 6.91(\mathrm{~d}, J=8.4 \mathrm{~Hz}, 2 \mathrm{H})$, $6.63(\mathrm{~m}, 1 \mathrm{H}), 6.38(\mathrm{~d}, J=7.6 \mathrm{~Hz}, 1 \mathrm{H}), 6.28-6.26(\mathrm{~m}, 1 \mathrm{H}), 3.66(\mathrm{~s}, 3 \mathrm{H}), 3.61(\mathrm{~s}, 3 \mathrm{H}), 3.08(\mathrm{dd}$, $J=9.3,7.3 \mathrm{~Hz}, 1 \mathrm{H}), 2.13(\mathrm{dd}, J=9.3,5.0 \mathrm{~Hz}, 1 \mathrm{H}), 1.81(\mathrm{dd}, J=7.3,5.0 \mathrm{~Hz}, 1 \mathrm{H})$. 
${ }^{13} \mathrm{C}$ NMR $\left(151 \mathrm{MHz}, \mathrm{CDCl}_{3}\right) \delta 173.9,159.3,137.6,134.2,133.6,131.1,129.0,121.4,120.7$, 113.7, 112.5, 55.2, 52.9, 36.9, 33.3, 20.7.

IR (ATR) 2920, 2850, 1717, 1250, 1046, 1011, 765, $720 \mathrm{~cm}^{-1}$.

HRMS-APCI $(\mathrm{m} / \mathrm{z})$ calc'd for $\mathrm{C}_{18} \mathrm{H}_{18} \mathrm{O}_{3} \mathrm{Br}[\mathrm{M}+\mathrm{H}]^{+}: 361.0434$, found 361.0434 .

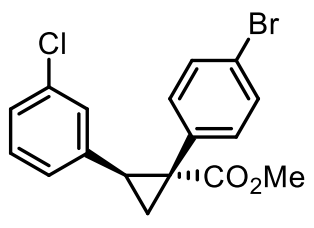

$73 \%(83 \%)$ yield

Methyl 1-(4-bromophenyl)-2-(3-chlorophenyl) cyclopropanecarboxylate

Following general procedure C, 9 was obtained as an off-white solid ( ${ }^{1} \mathbf{H}$ NMR yield: $83 \%$, isolated yield: $73 \%, 40.2 \mathrm{mg}$ ).

Rf: 0.20 (pentane/ethyl acetate 100:1)

m.p. $129-132{ }^{\circ} \mathrm{C}$.

${ }^{1} \mathbf{H}$ NMR $\left(600 \mathrm{MHz}, \mathrm{CDCl}_{3}\right) \delta 7.28(\mathrm{~d}, J=8.5 \mathrm{~Hz}, 2 \mathrm{H}), 7.08-7.04(\mathrm{~m}, 1 \mathrm{H}), 7.02-6.97(\mathrm{~m}, 1 \mathrm{H})$, $6.90(\mathrm{~d}, J=8.5 \mathrm{~Hz}, 2 \mathrm{H}), 6.87-6.85$ (m, $1 \mathrm{H}), 6.58-6.54$ (m, $1 \mathrm{H}), 3.66$ (s, $3 \mathrm{H}), 3.07$ (dd, $J=9.3$, $7.3 \mathrm{~Hz}, 1 \mathrm{H}), 2.14(\mathrm{dd}, J=9.3,5.1 \mathrm{~Hz}, 1 \mathrm{H}), 1.82(\mathrm{dd}, J=7.3,5.2 \mathrm{~Hz}, 1 \mathrm{H})$.

${ }^{13} \mathrm{C}$ NMR $\left(151 \mathrm{MHz}, \mathrm{CDCl}_{3}\right) \delta 173.6,138.3,134.0,133.6,133.6,131.2,129.2,128.6,126.9$, 126.0, 121.6, 52.9, 37.1, 32.7, 20.4.

IR (ATR) 3003, 2950, 1719, 1255, 1161, 1011, 764, $719 \mathrm{~cm}^{-1}$.

HRMS-APCI $(\mathrm{m} / z)$ calc'd for $\mathrm{C}_{17} \mathrm{H}_{15} \mathrm{O}_{2} \mathrm{BrCl}[\mathrm{M}+\mathrm{H}]^{+}:$364.9938, found 364.9939.
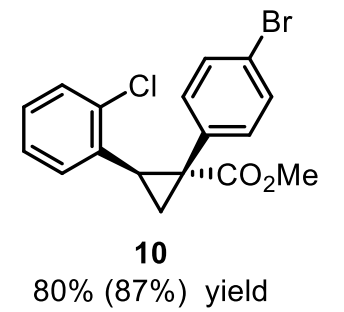

Methyl 1-(4-bromophenyl)-2-(2-chlorophenyl) cyclopropanecarboxylate

Following general procedure C, $\mathbf{1 0}$ was obtained as an off-white solid ( ${ }^{1} \mathbf{H}$ NMR yield: $87 \%$, isolated yield $44.0 \mathrm{mg}, 80 \%$ yield).

Rf: 0.20 (pentane/ethyl acetate 100:1).

m.p. $125-128^{\circ} \mathrm{C}$. 


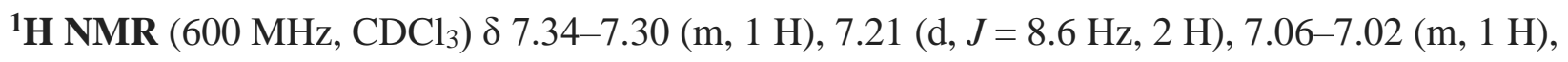
$6.98(\mathrm{~d}, J=8.6 \mathrm{~Hz}, 2 \mathrm{H}), 6.93-6.88(\mathrm{~m}, 1 \mathrm{H}), 6.55-6.51(\mathrm{~m}, 1 \mathrm{H}), 3.70(\mathrm{~s}, 3 \mathrm{H}), 3.37$ (dd, $J=\mathrm{Hz}$, $1 \mathrm{H}), 2.11(\mathrm{dd}, J=9.2,5.1 \mathrm{~Hz}, 1 \mathrm{H}), 1.98(\mathrm{dd}, J=7.6,5.3 \mathrm{~Hz}, 1 \mathrm{H})$.

${ }^{13}$ C NMR (151 MHz, $\left.\mathrm{CDCl}_{3}\right) \delta 173.7,136.2,134.1,134.0,133.0,131.0,129.3,128.2,127.7$, 126.5, 121.4, 52.9, 36.1, 31.6, 18.6.

IR (ATR) 3021, 2949, 1719, 1256, 1081, 1011, 769, $737 \mathrm{~cm}^{-1}$.

HRMS-APCI $(\mathrm{m} / z)$ calc'd for $\mathrm{C}_{17} \mathrm{H}_{15} \mathrm{O}_{2} \mathrm{BrCl}[\mathrm{M}+\mathrm{H}]^{+}:$364.9938, found 364.9939.

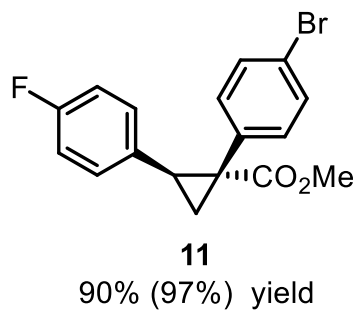

Methyl 1-(4-bromophenyl)-2-(4-fluorophenyl) cyclopropanecarboxylate

Following general procedure C, $\mathbf{1 1}$ was obtained as an off-white solid ( ${ }^{\mathbf{1}} \mathbf{H}$ NMR yield: $97 \%$, isolated yield: $90 \%, 50.6 \mathrm{mg}$ ).

Rf: 0.21 (pentane/ethyl acetate 100:1).

m.p. $104-106^{\circ} \mathrm{C}$.

${ }^{1} \mathbf{H}$ NMR $\left(500 \mathrm{MHz}, \mathrm{CDCl}_{3}\right) \delta 7.27(\mathrm{~d}, J=8.5 \mathrm{~Hz}, 2 \mathrm{H}), 6.88(\mathrm{~d}, J=8.5 \mathrm{~Hz}, 2 \mathrm{H}), 6.82-6.76(\mathrm{~m}$, 2 H), 6.75-6.70 (m, 2 H), 3.66 (s, 3 H), 3.09 (dd, $J=9.3,7.3, \mathrm{~Hz}, 1 \mathrm{H}), 2.14$ (dd, $J=9.3,5.1, \mathrm{~Hz}$, $1 \mathrm{H}), 1.78(\mathrm{dd}, J=7.3,5.0 \mathrm{~Hz}, 1 \mathrm{H})$.

${ }^{13}$ C NMR $\left(151 \mathrm{MHz}, \mathrm{CDCl}_{3}\right) \delta 173.8,133.9,133.7,131.2,129.6,129.5,121.5,115.1,115.0,52.9$, 36.8, 32.5, 20.6.

${ }^{19} \mathbf{F}$ NMR $\left(564 \mathrm{MHz}, \mathrm{CDCl}_{3}\right) \delta-115.9$

IR (ATR) 2951, 2924, 1719, 1513, 1257, 1160, 1012, $821 \mathrm{~cm}^{-1}$.

HRMS-APCI $(\mathrm{m} / z)$ calc'd for $\mathrm{C}_{17} \mathrm{H}_{15} \mathrm{O}_{2} \mathrm{BrF}[\mathrm{M}+\mathrm{H}]^{+}:$349.0234, found 349.0233 . 


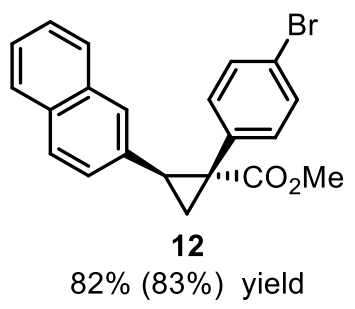

Methyl 1-(4-bromophenyl)-2-naphthylcyclopropanecarboxylate

Following general procedure C, 12 was obtained as an off-white solid ( ${ }^{1} \mathbf{H}$ NMR yield: 83\%, isolated yield: $82 \%, 46.1 \mathrm{mg}$ ).

Rf: 0.14 (pentane/ethyl acetate 100:1).

m.p. $118-120^{\circ} \mathrm{C}$.

${ }^{1} \mathbf{H}$ NMR $\left(600 \mathrm{MHz}, \mathrm{CDCl}_{3}\right) \delta$ 7.73-7.70 (m, $\left.1 \mathrm{H}\right), 7.65-7.62(\mathrm{~m}, 1 \mathrm{H}), 7.55(\mathrm{~m}, 1 \mathrm{H})$, 7.42-7.37 (m, 2 H), 7.36-7.34 (m, $1 \mathrm{H}), 7.21(\mathrm{~d}, J=8.5 \mathrm{~Hz}, 2 \mathrm{H}), 6.93(\mathrm{~d}, J=8.5 \mathrm{~Hz}, 2 \mathrm{H}), 6.81(\mathrm{~m}, 1 \mathrm{H})$, 3.69 (s, $3 \mathrm{H}), 3.28(\mathrm{dd}, J=9.3,7.3 \mathrm{~Hz}, 1 \mathrm{H}), 2.23(\mathrm{dd}, J=9.3,5.0 \mathrm{~Hz}, 1 \mathrm{H}), 1.98(\mathrm{dd}, J=8.0,5.0$ $\mathrm{Hz}, 1 \mathrm{H})$.

${ }^{13} \mathrm{C}$ NMR (151 MHz, $\left.\mathrm{CDCl}_{3}\right) \delta 173.9,134.0,133.7,133.7,133.1,132.3,131.1,127.7,127.7$. 127.6, 127.6, 127.3, 126.2, 126.0, 125.7, 121.4, 52.9, 37.1, 33.5, 20.7.

IR (ATR) 3052, 2949, 1717, 1256, 1068, 1011, 819, $749 \mathrm{~cm}^{-1}$.

HRMS-APCI $(\mathrm{m} / \mathrm{z})$ calc'd for $\mathrm{C}_{21} \mathrm{H}_{18} \mathrm{O}_{2} \mathrm{Br}[\mathrm{M}+\mathrm{H}]^{+}:$381.0485, found 381.0487 .

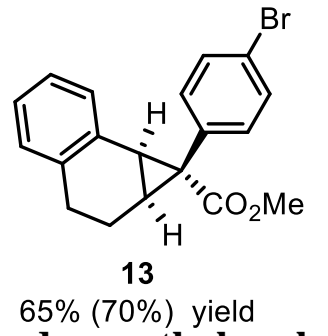

Methyl 1-(4-bromophenyl)-2,3-tetrahydronapthyl cyclopropanecarboxylate

Following general procedure C, $\mathbf{1 3}$ was obtained as an off-white solid ( ${ }^{1} \mathbf{H}$ NMR yield: $70 \%$, isolated yield: $35.0 \mathrm{mg}, 65 \%$ yield).

Rf: 0.17 (pentane/ethyl acetate 100:1).

m.p. $95-98{ }^{\circ} \mathrm{C}$.

${ }^{1} \mathbf{H}$ NMR $\left(600 \mathrm{MHz}, \mathrm{CDCl}_{3}\right) \delta 7.45-7.41(\mathrm{~m}, 1 \mathrm{H}), 7.23(\mathrm{~d}, J=8.6 \mathrm{~Hz}, 2 \mathrm{H}), 7.20-7.16(\mathrm{~m}, 1 \mathrm{H})$, 7.11-7.06 (m, $1 \mathrm{H}), 6.83$ (d, $J=8.6 \mathrm{~Hz}, 2 \mathrm{H}), 6.78-6.74$ (m, $1 \mathrm{H}), 3.62$ (s, $3 \mathrm{H}), 3.04$ (d, $J=9.3$, $1 \mathrm{H}), 2.58-2.53$ (m, $1 \mathrm{H}), 2.25-2.19$ (m, $1 \mathrm{H}), 2.16-2.09$ (m, $1 \mathrm{H}), 2.04-1.95$ (m, $1 \mathrm{H}), 1.11$ (ddd, $J=16.8,12.4,7.8,1 \mathrm{H})$. 
${ }^{13} \mathrm{C}$ NMR $\left(151 \mathrm{MHz}, \mathrm{CDCl}_{3}\right) \delta 173.6,135.2,134.0,132.7,132.7,131.3,130.3,128.7,126.8$, 126.2, 121.4, 52.8, 38.6, 31.1, 28.6, 25.5, 18.1.

IR (ATR) 3020, 2925, 1716, 1492, 1239, 1204, 1011, $751 \mathrm{~cm}^{-1}$.

HRMS-APCI $(\mathrm{m} / \mathrm{z})$ calc'd for $\mathrm{C}_{19} \mathrm{H}_{18} \mathrm{O}_{2} \mathrm{Br}[\mathrm{M}+\mathrm{H}]^{+}:$357.0485, found 357.0486.

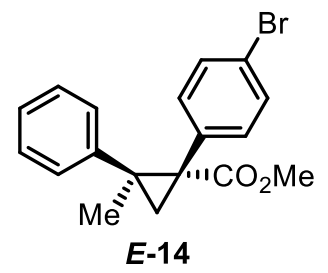

$75 \%(82 \%)$ yield, 8:1 d.r.

E-Methyl 1-(4-bromophenyl)-2-phenyl-2-methylcyclopropanecarboxylate

Following general procedure C, major diasteromer $\boldsymbol{E}-\mathbf{1 4}$ was obtained as an off-white solid $\left({ }^{\mathbf{1}} \mathbf{H}\right.$ NMR yield: $82 \%$, isolated yield: $75 \%, 38.9 \mathrm{mg} \mathrm{8:1} \mathrm{d.r.)} \mathrm{Both} \mathrm{diastereomers} \mathrm{have} \mathrm{previously}$ been reported with methyl phenyldiazoacetate ${ }^{19,20}$ providing sufficient evidence as to which diastereomer is the major.

Rf: 0.27 (pentane/ethyl acetate 100:1).

m.p. $97-101{ }^{\circ} \mathrm{C}$.

${ }^{1} \mathbf{H}$ NMR $\left(600 \mathrm{MHz}, \mathrm{CDCl}_{3}\right) \delta 7.15(\mathrm{~d}, J=8.6 \mathrm{~Hz}, 2 \mathrm{H}), 7.13-7.08$ (m, $\left.2 \mathrm{H}\right)$, 7.06-7.03 (m, $\left.3 \mathrm{H}\right)$, $7.01(\mathrm{~d}, J=8.6 \mathrm{~Hz}, 2 \mathrm{H}) 3.70(\mathrm{~s}, 3 \mathrm{H}), 2.06(\mathrm{~d}, J=5.7 \mathrm{~Hz}, 1 \mathrm{H}), 1.97$ (d, $J=5.7 \mathrm{~Hz}, 1 \mathrm{H}), 1.66$ (s, $3 \mathrm{H})$.

${ }^{13} \mathrm{C}$ NMR $\left(151 \mathrm{MHz}, \mathrm{CDCl}_{3}\right) \delta 171.8,140.7,135.8,133.2,130.6,128.1,128.0,126.6,120.9,52.6$, $41.5,34.3,22.7,22.3$.

IR (ATR) 2926, 2159, 1719, 1259, 1209, 1010, 799, $765 \mathrm{~cm}^{-1}$.

HRMS-APCI $(m / z)$ calc'd for $\mathrm{C}_{18} \mathrm{H}_{18} \mathrm{O}_{2} \mathrm{Br}[\mathrm{M}+\mathrm{H}]^{+}:$345.0485, found 345.0485.

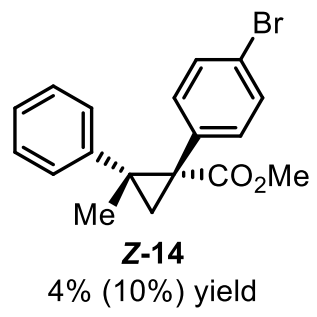

Z-Methyl 1-(4-bromophenyl)-2-phenyl-2-methylcyclopropanecarboxylate

In order to obtain enough material for characterization, general procedure $\mathrm{C}$ was performed on 300 $\mu$ mol scale with $\alpha$-methyl styrene. A $10 \%$ yield of the minor diastereomer $\mathbf{Z}-\mathbf{1 4}$ was observed by 
crude ${ }^{1} \mathrm{H}$ NMR using $300 \mu \mathrm{mol}$ of mesitylene as an internal standard. After performing column chromatography on the crude reaction (20:1 to 15:1 petroleum ether/ether) fractions containing $Z$ 14 were further purified by preparative TLC using 100:1 pentane/ethyl acetate as the mobile phase to isolate Z-14 as an off-white solid ( ${ }^{1} \mathbf{H}$ NMR yield: $10 \%$, isolated yield: $4 \%, 4.6 \mathrm{mg}$ ).

Rf: 0.31 (100:1 pentane/ethyl acetate)

m.p. $107-110{ }^{\circ} \mathrm{C}$.

${ }^{1} \mathbf{H}$ NMR $\left(600 \mathrm{MHz}, \mathrm{CDCl}_{3}\right) \delta 7.51(\mathrm{~d}, J=8.6 \mathrm{~Hz}, 2 \mathrm{H}), 7.39(\mathrm{~d}, J=8.6 \mathrm{~Hz}, 2 \mathrm{H}), 7.37-7.28(\mathrm{~m}$, $4 \mathrm{H}), 7.25-7.20(\mathrm{~m}, 1 \mathrm{H}), 3.19(\mathrm{~s}, 3 \mathrm{H}), 2.46(\mathrm{~d}, J=5.1 \mathrm{~Hz}, 1 \mathrm{H}), 1.42(\mathrm{~d}, J=5.1 \mathrm{~Hz}, 1 \mathrm{H}), 1.11$ (s, $3 \mathrm{H})$.

${ }^{13} \mathrm{C}$ NMR $\left(151 \mathrm{MHz}, \mathrm{CDCl}_{3}\right) \delta 171.1,142.7,136.1,133.3,131.3,128.5,128.4,126.9,121.5,52.1$, $40.5,36.5,26.2,24.1$.

IR (ATR) 2923, 2852, 1721, 1494, 1092, 1073, 772, $713 \mathrm{~cm}^{-1}$.

HRMS-APCI $(m / z)$ calc'd for $\mathrm{C}_{18} \mathrm{H}_{18} \mathrm{O}_{2} \mathrm{Br}[\mathrm{M}+\mathrm{H}]^{+}: 345.0485$, found 345.0482 .

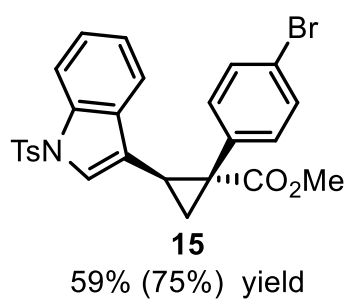

Methyl 1-(4-bromophenyl)-2-tosylvinylindolecyclopropanecarboxylate

Following general procedure C, 15 was obtained as an off-white solid ( ${ }^{1} \mathbf{H}$ NMR yield: $75 \%$, isolated yield: $59 \%, 46.0 \mathrm{mg}$ ).

Rf: 0.21 (4:1 petroleum ether/ether)

m.p. $116-120^{\circ} \mathrm{C}$.

${ }^{1}$ H NMR (600 MHz, $\left.\mathrm{CDCl}_{3}\right) \delta$ 7.84-7.80 (m, $\left.1 \mathrm{H}\right)$, 7.70-7.66 (m, $\left.1 \mathrm{H}\right), 7.34(\mathrm{~d}, J=8.4 \mathrm{~Hz}, 2 \mathrm{H})$, 7.32-7.29 (m, $2 \mathrm{H}), 7.24$ (d, J=8.2 Hz, $1 \mathrm{H}), 7.18$ (d, J=8.4 Hz, $1 \mathrm{H}), 6.90$ (d, J = 8.5 Hz, $1 \mathrm{H})$, $6.73(\mathrm{~s}, 1 \mathrm{H}) 3.72(\mathrm{~s}, 3 \mathrm{H}), 3.15(\mathrm{dd}, J=8.3,7.2 \mathrm{~Hz}, 1 \mathrm{H}), 2.40(\mathrm{~s}, 3 \mathrm{H}), 2.21(\mathrm{dd}, J=9.3,4.8 \mathrm{~Hz}$, $1.82(\mathrm{dd}, J=7.2,4.8 \mathrm{~Hz}, 1 \mathrm{H})$.

${ }^{13} \mathrm{C}$ NMR $\left(151 \mathrm{MHz}, \mathrm{CDCl}_{3}\right) \delta 173.7,145.0,134.9,134.8,134.2,133.2,131.2,131.1,130.0$, 126.6, 125.0, 123.4, 122.8, 121.4, 119.3, 118.6, 113.6, 53.0, 36.8, 23.7, 21.8, 20.3.

IR (ATR) 3112, 2980, 1718, 1367, 1188, 1092, 812, $746 \mathrm{~cm}^{-1}$.

HRMS-APCI $(m / z)$ calc'd for $\mathrm{C}_{26} \mathrm{H}_{23} \mathrm{O}_{4} \mathrm{BrNS}[\mathrm{M}+\mathrm{H}]^{+}:$524.0526, found 524.0530. 


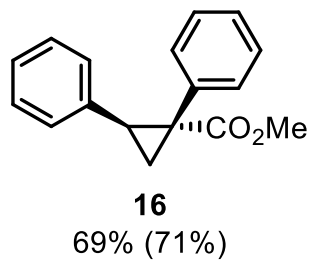

\section{Methyl 1,2-diphenylcyclopropanecarboxylate}

Following general procedure C, $\mathbf{1 6}$ was obtained as an off-white solid ( ${ }^{1} \mathbf{H}$ NMR yield: $71 \%$, isolated yield: $69 \%, 19.9 \mathrm{mg}$ ).

Rf: 0.26 (petroleum ether/ether 99:1).

${ }^{1} \mathbf{H}$ NMR $\left(600 \mathrm{MHz}, \mathrm{CDCl}_{3}\right) \delta$ 7.14-7.11 (m, $\left.3 \mathrm{H}\right), 7.07-7.04$ (m, $\left.3 \mathrm{H}\right), 7.03-7.01$ (m, $\left.2 \mathrm{H}\right), 6.78-$ $6.74(\mathrm{~m}, 2 \mathrm{H}), 3.67$ (s, $3 \mathrm{H}), 3.11(\mathrm{dd}, J=9.3,7.3 \mathrm{~Hz}, 1 \mathrm{H}), 2.14(\mathrm{dd}, J=9.2,4.9 \mathrm{~Hz}, 1 \mathrm{H}), 1.88$ (dd, $J=7.3,5.0 \mathrm{~Hz}, 1 \mathrm{H}$ ).

${ }^{13}$ C NMR $\left(151 \mathrm{MHz}, \mathrm{CDCl}_{3}\right) \delta 174.4,136.3,134.7,131.9,128.0,127.7,127.7,127.0,126.3,52.6$, $37.4,33.1,20.5$. All data matches data previously reported in the literature. ${ }^{19}$
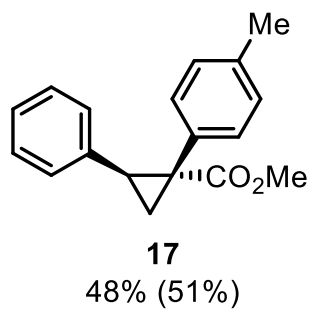

\section{Methyl 1-(4-methylphenyl)-2-phenylcyclopropanecarboxylate}

Following general procedure C, $\mathbf{1 7}$ was obtained as an off-white solid ( ${ }^{1} \mathbf{H}$ NMR yield: $51 \%$, isolated yield: $48 \%, 19.0 \mathrm{mg}$ ).

Rf: 0.26 (petroleum ether/ether $=99: 1$ ) .

${ }^{1} \mathbf{H}$ NMR $\left(400 \mathrm{MHz}, \mathrm{CDCl}_{3}\right) \delta 7.10-7.03(\mathrm{~m}, 3 \mathrm{H}), 6.93(\mathrm{~d}, J=8.1 \mathrm{~Hz}, 2 \mathrm{H}), 6.91(\mathrm{~d}, J=8.1 \mathrm{~Hz}$, 2 H), 6.81-6.75 (m, 2 H), 3.66 (s, 3 H), 3.08 (dd, $J=8.32 \mathrm{~Hz}, 7.72 \mathrm{~Hz}, 1 \mathrm{H}), 2.11$ (dd, $J=9.4 \mathrm{~Hz}$, $4.8 \mathrm{~Hz}, 1 \mathrm{H}), 1.84(\mathrm{dd}, J=7.5,5.1, \mathrm{~Hz}, 1 \mathrm{H})$.

${ }^{13} \mathbf{C}$ NMR $\left(151 \mathrm{MHz}, \mathrm{CDCl}_{3}\right) \delta 174.6,136.6,136.5,131.7,131.6,128.4,128.1,127.7,126.2,52.6$, $37.0,33.1,21.2,20.6$. All data matches data previously reported in the literature. ${ }^{19}$ 


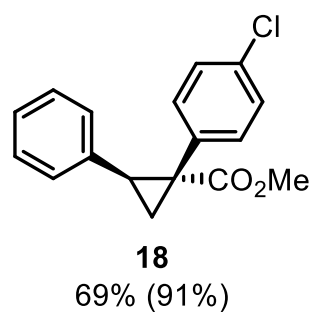

Methyl 1-(4-chlorophenyl)-2-phenylcyclopropanecarboxylate

Following general procedure C, 18 was obtained an off-white solid ( ${ }^{\mathbf{1}} \mathbf{H}$ NMR yield: $91 \%$, isolated yield: $69 \%, 29.9 \mathrm{mg}$ ).

Rf: 0.20 (pentane/ethyl acetate 100:1).

${ }^{1} \mathbf{H}$ NMR $\left(600 \mathrm{MHz}, \mathrm{CDCl}_{3}\right) \delta 7.10(\mathrm{~d}, J=8.5 \mathrm{~Hz}, 2 \mathrm{H}), 7.09-7.07(\mathrm{~m}, 3 \mathrm{H}), 6.95(\mathrm{~d}, J=8.5 \mathrm{~Hz}$, 2 H), 6.80-6.75 (m, 2 H) 3.67 (s, 3 H), $3.11(\mathrm{dd}, J=9.4,7.4 \mathrm{~Hz}, 1 \mathrm{H}), 2.14$ (dd, $J=9.3,5.0 \mathrm{~Hz}, 1$ $\mathrm{H}), 1.84(\mathrm{dd}, J=7.3,5.0 \mathrm{~Hz}, 1 \mathrm{H})$.

${ }^{13} \mathrm{C}$ NMR $\left(151 \mathrm{MHz}, \mathrm{CDCl}_{3}\right) \delta 173.8,135.8,133.4,133.2,132.9,128.0,127.9,127.9,126.5,52.7$, $36.7,33.2,20.3$. All data matches data previously reported in the literature. ${ }^{22}$

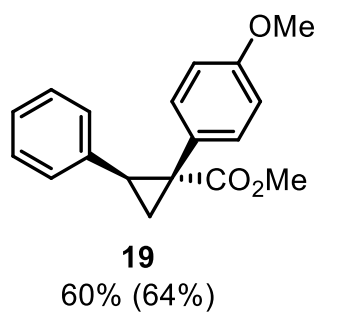

Methyl 1-(4-methoxyphenyl)-2-phenylcyclopropanecarboxylate

Following general procedure C, 19 was obtained as an off-white solid ( ${ }^{1} \mathbf{H}$ NMR yield: 64\%, isolated yield: $60 \%, 25.6 \mathrm{mg}$ ).

Rf: 0.14 (pentane/ethyl acetate 30:1).

${ }^{1}$ H NMR $\left(500 \mathrm{MHz}, \mathrm{CDCl}_{3}\right) \delta$ 7.10-7.04 (m, $\left.3 \mathrm{H}\right), 6.93(\mathrm{~d}, J=8.8 \mathrm{~Hz}, 2 \mathrm{H}), 6.80-6.75(\mathrm{~m}, 2 \mathrm{H})$, $6.67(\mathrm{~d}, J=8.8 \mathrm{~Hz}, 2 \mathrm{H}), 3.72(\mathrm{~s}, 3 \mathrm{H}), 3.66$ (s, $3 \mathrm{H}), 3.07$ (dd, $J=9.3,7.3 \mathrm{~Hz}, 1 \mathrm{H}), 2.12$ (dd, $J=$ 9.3, $4.8 \mathrm{~Hz}, 1 \mathrm{H}), 1.82(\mathrm{dd}, J=7.3,4.8 \mathrm{~Hz}, 1 \mathrm{H})$.

${ }^{13} \mathrm{C}$ NMR $\left(151 \mathrm{MHz}, \mathrm{CDCl}_{3}\right) \delta 174.6,158.4,136.5,132.9,128.1,127.7,126.8,126.2,113.1,55.1$, $52.6,36.7,33.2,20.8$. All data matches data previously reported in the literature. ${ }^{22}$ 


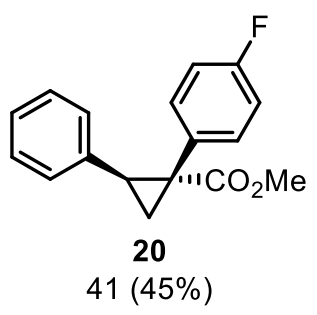

\section{Methyl 1-(4-fluorophenyl)-2-phenylcyclopropanecarboxylate}

Following general procedure C, 20 was obtained as an off-white solid ( ${ }^{1} \mathbf{H}$ NMR yield: $45 \%$, isolated yield: $41 \%, 16.6 \mathrm{mg})$.

Rf: 0.42 (9:1 petroleum ether/ether)

${ }^{1}$ H NMR (600 MHz, CDCl $)$ א 7.10-7.04 (m, 3 H), 7.00-6.94 (m, 2 H), 6.84-6.78 (m, 2 H), 6.77$6.73(\mathrm{~m}, 2 \mathrm{H}), 3.67$ (s, $3 \mathrm{H}), 3.10(\mathrm{dd}, J=9.4,7.4 \mathrm{~Hz}, 1 \mathrm{H}), 2.15(\mathrm{dd}, J=9.3,5.0 \mathrm{~Hz}, 1 \mathrm{H}), 1.84$ (dd, $J=7.3,5.0 \mathrm{~Hz}, 1 \mathrm{H}$ ).

${ }^{13} \mathrm{C}$ NMR $\left(151 \mathrm{MHz}, \mathrm{CDCl}_{3}\right) \delta 174.3,162.7,161.0,136.1,133.6(\mathrm{~d}, J=8.2 \mathrm{~Hz}), 130.7(\mathrm{~d}, J=$ $3.8 \mathrm{~Hz}), 128.1(\mathrm{~d}, J=28.3 \mathrm{~Hz}), 126.6,114.8(\mathrm{~d}, J=21.2 \mathrm{~Hz}), 52.8,36.7,33.3,20.7$.

${ }^{19}$ F NMR $\left(564 \mathrm{MHz}, \mathrm{CDCl}_{3}\right) \delta-115.0$

All data matches data previously reported in the literature. ${ }^{23}$

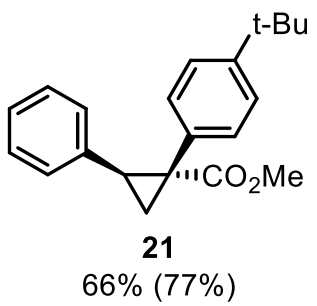

\section{Methyl 1-(4-tert-butylphenyl)-2-phenylcyclopropanecarboxylate}

Following general procedure C, 21 was obtained as an off-white solid ( ${ }^{\mathbf{H}} \mathbf{H}$ NMR yield: 77\%, isolated yield: $66 \%, 30.3 \mathrm{mg}$ ).

Rf: 0.29 (pentane/ethyl acetate 100:1).

${ }^{1} \mathbf{H}$ NMR $\left(600 \mathrm{MHz}, \mathrm{CDCl}_{3}\right) \delta 7.12(\mathrm{~d}, J=8.5 \mathrm{~Hz}, 2 \mathrm{H}), 7.05-7.01(\mathrm{~m}, 3 \mathrm{H}), 6.93(\mathrm{~d}, J=8.5 \mathrm{~Hz}$, $2 \mathrm{H}), 6.75-6.70(\mathrm{~m}, 2 \mathrm{H}), 3.67$ (s, $3 \mathrm{H}), 3.06(\mathrm{dd}, J=9.3,7.3 \mathrm{~Hz}, 1 \mathrm{H}), 2.13$ (dd, $J=9.4,4.9 \mathrm{~Hz}$, $1 \mathrm{H}), 1.84$ (dd, $J=7.3,4.9 \mathrm{~Hz}, 1 \mathrm{H}), 1.23$ (s, $9 \mathrm{H})$.

${ }^{13} \mathrm{C}$ NMR $\left(151 \mathrm{MHz}, \mathrm{CDCl}_{3}\right) \delta 174.6,149.7,148.9,136.5,131.4,128.0,127.5,126.1,124.6,52.6$, $37.0,34.4,33.2,31.3,20.7$. All data matches data previously reported in the literature. ${ }^{20}$ 


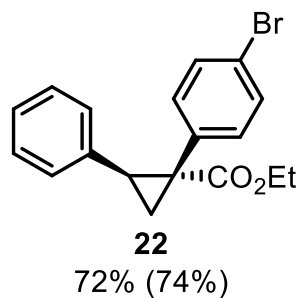

Ethyl 1-(4-bromophenyl)-2-phenylcyclopropanecarboxylate

Following general procedure C, 22 was obtained as an off-white solid ( ${ }^{\mathbf{1}} \mathbf{H}$ NMR yield: $74 \%$, isolated yield: $72 \%, 37.1 \mathrm{mg}$ ).

Rf: 0.24 (pentane/ethyl acetate 100:1).

${ }^{1} \mathbf{H}$ NMR $\left(600 \mathrm{MHz} \mathrm{CDCl}_{3}\right) \delta 7.24(\mathrm{~d}, J=8.4 \mathrm{~Hz}, 2 \mathrm{H}), 7.12-7.06(\mathrm{~m}, 3 \mathrm{H}), 6.89$ (d, $J=8.4 \mathrm{~Hz}$, $2 \mathrm{H}), 6.80-6.75$ (m, 2 H), 4.20-4.07 (m, 2 H), 3.09 (dd, J = 9.4, 7.3 Hz, 1 H), 2.13 (dd, 9.3, 5.0 $\mathrm{Hz}, 1 \mathrm{H}), 1.83$ (dd, $J=7.3,4.9 \mathrm{~Hz}, 1 \mathrm{H}), 1.18(\mathrm{t}, J=7.13 \mathrm{~Hz}, 3 \mathrm{H})$.

${ }^{13} \mathrm{C}$ NMR $\left(151 \mathrm{MHz}, \mathrm{CDCl}_{3}\right) \delta 173.2,135.9,134.1,133.5,130.8,128.0,127.9,126.5,121.1,61.4$, $36.9,32.9,20.0,14.1$ All data matches data previously reported in the literature. ${ }^{24}$

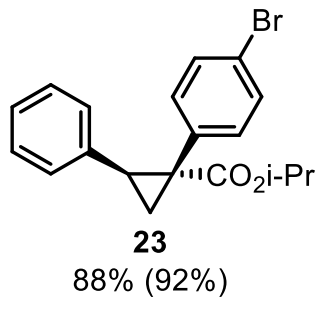

Isopropyl 1-(4-bromophenyl)-2-phenylcyclopropanecarboxylate

Following general procedure C, $3 \mathbf{t}$ was obtained as an off-white solid ( ${ }^{1} \mathbf{H}$ NMR yield: $92 \%$, isolated yield: $88 \%, 49.5 \mathrm{mg}$ ).

Rf: 0.32 (pentane/ethyl acetate 100:1).

m.p. $67-70{ }^{\circ} \mathrm{C}$.

${ }^{1} \mathbf{H}$ NMR (400 MHz, $\left.\mathrm{CDCl}_{3}\right) \delta 7.23(\mathrm{~d}, J=8.5 \mathrm{~Hz}, 2 \mathrm{H}), 7.11-7.06(\mathrm{~m}, 3 \mathrm{H}), 6.88(\mathrm{~d}, J=8.5 \mathrm{~Hz}$, 2 H), 6.80-6.75 (m, 2 H), 4.99 (sept, $J=6.3 \mathrm{~Hz}, 1 \mathrm{H}$ ), 3.07 (dd, $J=9.3,7.2 \mathrm{~Hz}, 1 \mathrm{H}), 2.11$ (dd, $J$ = 9.3, 4.9 Hz, $1 \mathrm{H}), 1.81(\mathrm{dd}, J=7.3,5.0 \mathrm{~Hz}, 1 \mathrm{H}), 1.16(\mathrm{~m}, 6 \mathrm{H})$.

${ }^{13} \mathrm{C}$ NMR $\left(151 \mathrm{MHz}, \mathrm{CDCl}_{3}\right) \delta 172.7,136.2,134.4,133.7,130.9,128.2,128.0,126.6,121.1,68.9$, $37.3,32.9,21.8,19.9$.

IR (ATR) 2961, 2924, 1710, 1258, 1087, 1012, 797, $713 \mathrm{~cm}^{-1}$.

HRMS $(m / z)$ calc'd for $\mathrm{C}_{19} \mathrm{H}_{20} \mathrm{O}_{2} \mathrm{Br}[\mathrm{M}+\mathrm{H}]^{+}:$359.0641, found 359.0641. 


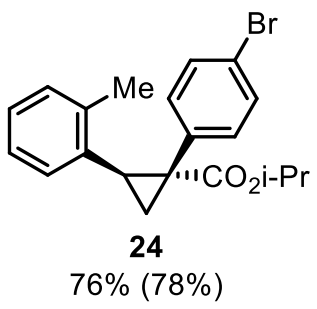

Isopropyl 1-(4-bromophenyl)-2-(2-methylphenyl) cyclopropanecarboxylate

Following general procedure C, 24 was obtained as an off-white solid ( ${ }^{\mathbf{1}} \mathbf{H}$ NMR yield: $78 \%$, isolated yield: $76 \%, 42.5 \mathrm{mg})$.

Rf: 0.48 (18:1 petroleum ether/ether)

m.p. $110-114^{\circ} \mathrm{C}$.

${ }^{1} \mathbf{H}$ NMR $\left(600 \mathrm{MHz}, \mathrm{CDCl}_{3}\right) \delta 7.17(\mathrm{~d}, J=8.5 \mathrm{~Hz}, 2 \mathrm{H}), 7.12-7.08$ (m, $\left.1 \mathrm{H}\right), 7.02-=-6.98$ (m, 1 H), 6.86-6.83 (m, $1 \mathrm{H}), 6.82(\mathrm{~d}, J=8.5 \mathrm{~Hz}, 1 \mathrm{H}), 4.64$ (sept, $J=6.3 \mathrm{~Hz}, 1 \mathrm{H}), 3.08$ (dd, $J=9.2$, $7.6 \mathrm{~Hz}, 1 \mathrm{H}), 2.45(\mathrm{~s}, 3 \mathrm{H}) 2.06(\mathrm{dd}, J=9.2,5.0 \mathrm{~Hz}, 1 \mathrm{H}), 1.98(\mathrm{dd}, J=7.5,5.0 \mathrm{~Hz}, 1 \mathrm{H}), 1.20(\mathrm{~d}$, $6.2 \mathrm{~Hz}, 3 \mathrm{H}), 1.17$ (d, $6.2 \mathrm{~Hz}, 3 \mathrm{H})$.

${ }^{13} \mathrm{C}$ NMR $\left(151 \mathrm{MHz}, \mathrm{CDCl}_{3}\right) \delta 172.9,137.8,134.5,133.0,130.7,129.0,126.8,125.8,125.7$, $120.9,68.8,36.3,31.2,21.8,20.1,18.1$.

IR (ATR) 2978, 2933, 1711, 1373, 1257, 819, 769, $737 \mathrm{~cm}^{-1}$.

HRMS (m/z) calc'd for $\mathrm{C}_{20} \mathrm{H}_{22} \mathrm{O}_{2} \mathrm{Br}[\mathrm{M}+\mathrm{H}]^{+}: 373.0798$, found 373.0799.

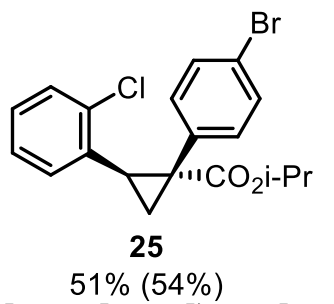

\section{Isopropyl 1-(4-bromophenyl)-2-(2-chlorophenyl) cyclopropanecarboxylate}

Following general procedure C, 25 was obtained as an off-white oil ( ${ }^{1}$ H NMR yield: 54\%, isolated yield: $51 \%, 30.2 \mathrm{mg}$ ).

Rf: 0.47 (9:1 petroleum ether/ether).

${ }^{1} \mathbf{H}$ NMR $\left(600 \mathrm{MHz}, \mathrm{CDCl}_{3}\right) \delta$ 7.33-7.29 (m, $\left.1 \mathrm{H}\right), 7.19(\mathrm{~d}, J=8.5 \mathrm{~Hz}, 2 \mathrm{H}), 7.05-7.01(\mathrm{~m}, 1 \mathrm{H})$, $6.97(\mathrm{~d}, J=8.5 \mathrm{~Hz}, 2 \mathrm{H}), 6.92-6.88(\mathrm{~m}, 1 \mathrm{H}), 6.55-6.52$ (m, $1 \mathrm{H}), 5.05$ (sept, $J=6.2 \mathrm{~Hz}, 1 \mathrm{H}), 3.31$ $(\mathrm{dd}, J=9.1,7.5 \mathrm{~Hz}, 1 \mathrm{H}), 2.10(\mathrm{dd}, J=9.1,5.1 \mathrm{~Hz}, 1 \mathrm{H}), 1.96(\mathrm{dd}, J=7.5,5.1 \mathrm{~Hz}, 1 \mathrm{H}), 1.22(\mathrm{~d}$, $J=6.3 \mathrm{~Hz}, 3 \mathrm{H}) 1.18(\mathrm{~d}, J=6.3,3 \mathrm{H})$. 
${ }^{13} \mathrm{C}$ NMR $\left(151 \mathrm{MHz}, \mathrm{CDCl}_{3}\right) \delta 172.5,136.2,134.3,134.1,133.0,130.8,129.3,128.1,127.6$, 126.5, 121.1, 68.9, 36.4, 31.5, 21.8, 21.8, 17.7.

IR (ATR) 2978, 2933, 1712, 1488, 1255, 820, 769, $737 \mathrm{~cm}^{-1}$.

HRMS $(\mathrm{m} / z)$ calc'd for $\mathrm{C}_{19} \mathrm{H}_{19} \mathrm{O}_{2} \mathrm{BrCl}[\mathrm{M}+\mathrm{H}]^{+}: 393.0251$, found 393.0253 .

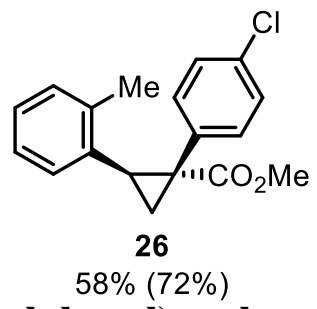

Methyl 1-(4-chlorophenyl)-2-(2-methylphenyl) cyclopropanecarboxylate

Following general procedure C, 26 was obtained as an off-white solid ( ${ }^{1} \mathbf{H}$ NMR yield: $72 \%$, isolated yield: $58 \%, 26.1 \mathrm{mg}$ ).

Rf: 0.37 (18:1 petroleum ether/ether)

m.p. $140-144^{\circ} \mathrm{C}$.

${ }^{1} \mathbf{H}$ NMR $\left(600 \mathrm{MHz}, \mathrm{CDCl}_{3}\right) \delta$ 7.13-7.09 (m, $\left.1 \mathrm{H}\right), 7.04(\mathrm{~d}, J=8.5 \mathrm{~Hz}, 2 \mathrm{H}), 7.02-6.98(\mathrm{~m}, 1 \mathrm{H})$, 6.89 (d, $J=8.5 \mathrm{~Hz}, 2 \mathrm{H}), 6.85-6.81$ (m, $1 \mathrm{H}), 6.41-6.37$ (m, $1 \mathrm{H}), 3.69$ (s, $3 \mathrm{H}), 3.14$ (dd, $J=9.2$, $7.5 \mathrm{~Hz}, 1 \mathrm{H}), 2.46(\mathrm{~s}, 3 \mathrm{H}), 2.07$ (dd, $J=9.2,5.0 \mathrm{~Hz}, 1 \mathrm{H}) 2.01(\mathrm{dd}, J=7.5,5.0 \mathrm{~Hz}, 1 \mathrm{H})$.

${ }^{13} \mathbf{C}$ NMR $\left(151 \mathrm{MHz}, \mathrm{CDCl}_{3}\right) \delta 174.2,137.9,133.9,133.8,132.9,132.6,129.9,128.0,126.8$, $125.8,125.7,52.9,35.8,31.3,20.2,19.0$.

IR (ATR) 2923, 2850, 1718, 1494, 1258, 795, 772, $730 \mathrm{~cm}^{-1}$.

HRMS $(\mathrm{m} / \mathrm{z})$ calc'd for $\mathrm{C}_{18} \mathrm{H}_{18} \mathrm{O}_{2} \mathrm{Cl}[\mathrm{M}+\mathrm{H}]^{+}:$301.0990, found 301.0990.

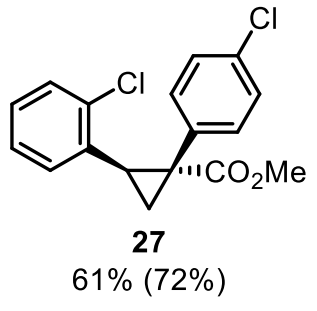

Methyl 1-(4-chlorophenyl)-2-(2-chlorophenyl) cyclopropanecarboxylate

Following general procedure C, 27 was obtained as an off-white solid ( ${ }^{1} \mathbf{H}$ NMR yield: $72 \%$, isolated yield: $61 \%, 29.4 \mathrm{mg}$ ).

Rf: 0.40 ( $18: 1$ petroleum ether/ether)

m.p. $113-117^{\circ} \mathrm{C}$. 
${ }^{1} \mathbf{H}$ NMR $\left(600 \mathrm{MHz}, \mathrm{CDCl}_{3}\right) \delta$ 7.34-7.30 (m, $\left.1 \mathrm{H}\right)$, 7.09-7.01 (m, $\left.5 \mathrm{H}\right), 6.93-6.89$ (m, $\left.1 \mathrm{H}\right), 6.55-$ $6.51(\mathrm{~m}, 1 \mathrm{H}), 3.70(\mathrm{~s}, 3 \mathrm{H}), 3.37$ (dd, $J=9.1,7.6 \mathrm{~Hz}, 1 \mathrm{H}), 2.11(\mathrm{dd}, J=9.1,5.1 \mathrm{~Hz}, 1 \mathrm{H}), 1.99$ (dd, $J=7.6,5.1 \mathrm{~Hz}, 1 \mathrm{H})$.

${ }^{13} \mathrm{C}$ NMR $\left(151 \mathrm{MHz}, \mathrm{CDCl}_{3}\right) \delta 173.8,136.2,134.0,133.5,133.1,132.6,129.3,128.2,128.1$, 127.7, 126.5, 52.9, 35.9, 31.6, 18.6.

IR (ATR) 2980, 2846, 1720, 1257, 1092, 795, 760, $738 \mathrm{~cm}^{-1}$.

HRMS $(m / z)$ calc'd for $\mathrm{C}_{17} \mathrm{H}_{15} \mathrm{O}_{2} \mathrm{Cl}_{2}[\mathrm{M}+\mathrm{H}]^{+}:$321.0444, found 321.0444

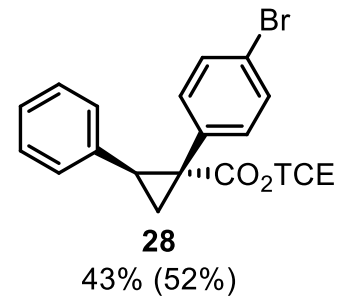

2,2,2-trichloroethyl 1-(4-bromophenyl)-2-phenylcyclopropanecarboxylate

Following general procedure C, 28 was obtained as an off-white solid ( ${ }^{1} \mathbf{H}$ NMR yield: $52 \%$, isolated yield: $43 \%, 28.7 \mathrm{mg}$ ).

Rf: 0.39 (18:1 petroleum ether/ether).

${ }^{1} \mathbf{H}$ NMR $\left(600 \mathrm{MHz}, \mathrm{CDCl}_{3}\right) \delta 7.25(\mathrm{~d}, J=8.5 \mathrm{~Hz}, 2 \mathrm{H}), 7.13-7.09(\mathrm{~m}, 3 \mathrm{H}), 6.93(\mathrm{~d}, J=8.5 \mathrm{~Hz}$, $2 \mathrm{H}), 6.82-6.77(\mathrm{~m}, 2 \mathrm{H}), 4.83$ (d, $J=11.9 \mathrm{~Hz}, 1 \mathrm{H}), 4.64$ (d, $J=11.9 \mathrm{~Hz}, 1 \mathrm{H}), 3.22$ (dd, $J=7.5$, $2.0 \mathrm{~Hz}, 1 \mathrm{H}), 2.28$ (dd, $J=5.2,4.1 \mathrm{~Hz}, 1 \mathrm{H}), 1.97$ (dd, $J=5.2,2.3 \mathrm{~Hz}, 1 \mathrm{H})$.

${ }^{13} \mathrm{C}$ NMR $\left(151 \mathrm{MHz}, \mathrm{CDCl}_{3}\right) \delta 171.7,135.3,133.8,133.1,131.1,128.2,128.2,127.0,121.7,74.5$, $36.7,34.1,20.3$. All data matches data previously reported in the literature. ${ }^{25}$

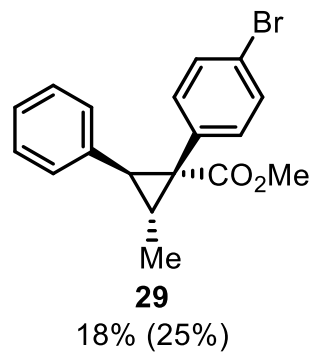

Methyl 1-(4-bromophenyl)-2-phenyl-3-methylcyclopropanecarboxylate

Following general procedure C, 29 was obtained as an off-white solid ( ${ }^{1} \mathbf{H}$ NMR yield: $25 \%$, isolated yield: $18 \%, 9.4 \mathrm{mg}$ ).

Rf: 0.37 (petroleum ether/ether 18:1).

m.p. $117-120^{\circ} \mathrm{C}$. 
${ }^{1} \mathbf{H}$ NMR $\left(600 \mathrm{MHz}, \mathrm{CDCl}_{3}\right) \delta 7.25(\mathrm{~d}, J=8.1 \mathrm{~Hz}, 2 \mathrm{H}), 7.11-7.05(\mathrm{~m}, 3 \mathrm{H}), 6.86(\mathrm{~d}, J=8.1 \mathrm{~Hz}$, 2 H), 6.78-6.74 (m, 2 H), 3.65 (s, 3 H), 3.08 (d, $J=7.6 \mathrm{~Hz}, 1 \mathrm{H}), 2.20(\mathrm{~m}, 1 \mathrm{H}), 1.47$ (d, $J=6.2$ $\mathrm{Hz}, 1 \mathrm{H})$.

${ }^{13}$ C NMR $\left(151 \mathrm{MHz}, \mathrm{CDCl}_{3}\right) \delta 171.9,136.7,135.7,133.2,131.1,128.1,128.0,126.4,121.1,52.6$, $42.8,38.0,27.6,12.9$.

IR (ATR) 2950, 2135, 1716, 1505, 1071, 1010, 750, $716 \mathrm{~cm}^{-1}$

HRMS-APCI (m/z) calc'd for $\mathrm{C}_{18} \mathrm{H}_{18} \mathrm{O}_{2} \mathrm{Br}[\mathrm{M}+\mathrm{H}]^{+}: 345.0485$, found 345.0483 .

Mass balance: Styrene remaining by NMR: $30 \%$.

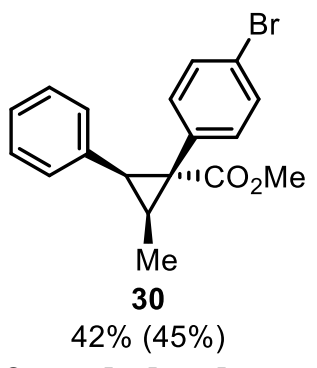

Methyl 1-(4-bromophenyl)-2-phenyl-3-methylcyclopropanecarboxylate

Following general procedure C, 30 was obtained as an off-white solid ( ${ }^{1} \mathbf{H}$ NMR yield: $45 \%$, isolated yield: $42 \%, 22.0 \mathrm{mg}$ ).

Rf: 0.28 (petroleum ether/ether 18:1).

m.p. $128-130{ }^{\circ} \mathrm{C}$.

${ }^{1} \mathbf{H}$ NMR $\left(600 \mathrm{MHz}, \mathrm{CDCl}_{3}\right) \delta 7.39(\mathrm{~d}, J=8.5 \mathrm{~Hz}, 2 \mathrm{H}), 7.19-7.13(\mathrm{~m}, 3 \mathrm{H}), 6.90(\mathrm{~d}, J=8.5 \mathrm{~Hz}$, $2 \mathrm{H}), 6.83-6.78(\mathrm{~m}, 2 \mathrm{H}), 3.62(\mathrm{~s}, 1 \mathrm{H}), 3.10(\mathrm{~d}, J=10.2 \mathrm{~Hz}, 1 \mathrm{H}), 2.38(\mathrm{dq}, J=6.8,3.4 \mathrm{~Hz}, 1 \mathrm{H})$. $1.25(\mathrm{~d}, J=6.8 \mathrm{~Hz}, 1 \mathrm{H})$.

${ }^{13}$ C NMR $\left(151 \mathrm{MHz}, \mathrm{CDCl}_{3}\right) \delta 175.1,135.9,135.0,131.6,131.3,130.4,127.8,126.3,121.7,52.9$, 37.6, 36.7, 27.9, 11.0.

IR (ATR) 3029, 2949, 1717, 1244, 1225, 1054, 716, $702.8 \mathrm{~cm}^{-1}$.

HRMS-APCI (m/z) calc'd for $\mathrm{C}_{18} \mathrm{H}_{18} \mathrm{O}_{2} \mathrm{Br}[\mathrm{M}+\mathrm{H}]^{+}:$345.0485, found 345.0483.

Mass balance: Styrene remaining by ${ }^{1} \mathrm{H}$ NMR: $20 \%$. 


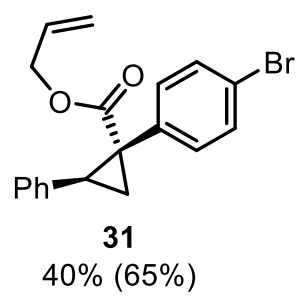

\section{Allyl 1-(4-bromophenyl)-2-phenylcyclopropanecarboxylate}

Following general procedure C, $\mathbf{3 1}$ was obtained as a low melting clear solid $\left({ }^{1} \mathbf{H}\right.$ NMR yield: $65 \%$, isolated yield: $40 \%, 21.5 \mathrm{mg}$ ).

Rf: 0.25 (petroleum ether/ether 18:1).

${ }^{1} \mathrm{H}$ NMR $\left(400 \mathrm{MHz}, \mathrm{CDCl}_{3}\right) \delta 7.25(\mathrm{~d}, J=8.1 \mathrm{~Hz}, 2 \mathrm{H}), 7.13-7.06(\mathrm{~m}, 3 \mathrm{H}), 6.90(\mathrm{~d}, J=8.1 \mathrm{~Hz}$, 2 H), 6.81-6.75 (m, 2 H), 5.82 (ddd, $J=16.0,10.6,5.3 \mathrm{~Hz}, 1 \mathrm{H}$ ), 5.20-5.09 (m, 2H), 4.57 (dq, $J$ $=13.8,5.2 \mathrm{~Hz}, 2 \mathrm{H}), 3.12(\mathrm{dd}, J=9.3,7.4 \mathrm{~Hz}), 2.16(\mathrm{dd}, J=9.3,5.0 \mathrm{~Hz}, 1 \mathrm{H}), 1.85(\mathrm{dd}, J=7.4$, $5.2 \mathrm{~Hz}, 1 \mathrm{H})$.

${ }^{13}$ C NMR $\left(151 \mathrm{MHz}, \mathrm{CDCl}_{3}\right) \delta 173.0,136.0,134.0,134.0,133.7,132.0,131.0,128.2,128.1$, 126.7, 121.3, 117.7, 65.9, 37.0, 33.3, 20.4 .

IR (ATR) 2915, 2847, 1715, 1488, 1161, 1011, 769, $713 \mathrm{~cm}^{-1}$

HRMS-APCI $(\mathrm{m} / \mathrm{z})$ calc'd for $\mathrm{C}_{19} \mathrm{H}_{18} \mathrm{O}_{2} \mathrm{Br}[\mathrm{M}+\mathrm{H}]^{+}: 357.0485$, found 357.0484 .

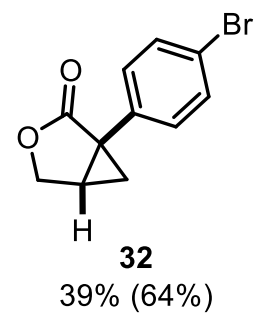

1-(4-bromophenyl)-2-oxo-3-oxabicyclo[3.1.0] hexane (32) was observed in $27 \%$ yield by ${ }^{1} \mathrm{H}$ NMR in the reaction shown in eq 3 . Attempts to isolate this product from the reaction mixture proved unsuccessful. Instead, $\mathbf{3 2}$ was synthesized independently using the following procedure:

In a nitrogen-filled glove box, allyl 2-(4-bromophenyl)-2-diazoacetate (26.0 mg, $92.5 \mu \mathrm{mol}, 1$ equiv) was weighed into a $10-\mathrm{mL}$ reaction tube with a stir bar. The tube was capped with a Teflon-coated septa cap and removed from the glovebox. To this was added 1,2-dichloroethane $(6.0 \mathrm{~mL}, 0.015 \mathrm{M})$. The reaction was heated at $110^{\circ} \mathrm{C}$ for $20 \mathrm{~h}$. The reaction was cooled to room temperature and concentrated under reduced pressure. Mesitylene $(92.5 \mu \mathrm{mol})$ was added. A $64 \%$ yield of 32 was observed by crude ${ }^{1} \mathrm{H}$ NMR. The crude reaction mixture was dry loaded onto silica and purified via column chromatography (2:1 to 1:1 petroleum ether/ether gradient) to isolate 32. 
32 was obtained as a clear oil ( ${ }^{1}$ H NMR yield: $64 \%$, isolated yield: $39 \%$ (9.1 mg).

Rf: 0.10 (1:1 petroleum ether/ether)

${ }^{1}$ H NMR $\left(600 \mathrm{MHz}, \mathrm{CDCl}_{3}\right) \delta 7.48(\mathrm{~d}, J=8.6 \mathrm{~Hz}, 2 \mathrm{H}), 7.30(\mathrm{~d}, J=8.6 \mathrm{~Hz}, 2 \mathrm{H}), 4.47$ (dd, $J=$ 9.3, 4.6 Hz, $1 \mathrm{H}), 4.30(\mathrm{~d}, J=9.31 \mathrm{H}), 2.57(\mathrm{dt}, J=7.8,4.6 \mathrm{~Hz}, 1 \mathrm{H}), 1.61(\mathrm{dd}, J=7.9,4.9 \mathrm{~Hz}, 1$ H), 1.39 (t, $J=4.8 \mathrm{~Hz}, 1 \mathrm{H})$.

${ }^{13}$ C NMR (151 MHz, $\left.\mathrm{CDCl}_{3}\right) \delta 175.6,133.3,131.9,130.1,121.9,68.2,31.3,25.2,20.6$.

IR (ATR) 2917, 2880, 1760, 1493, 1077, 1002, 834, $717 \mathrm{~cm}^{-1}$.

HRMS-APCI $(\mathrm{m} / z)$ calc'd for $\mathrm{C}_{11} \mathrm{H}_{10} \mathrm{BrO}_{2}[\mathrm{M}+\mathrm{H}]^{+}:$252.9859, found 252.9857. 


\section{Control experiment for cyclopropane stability in the presence of $B\left(C_{6} F_{5}\right)_{3}$ :}

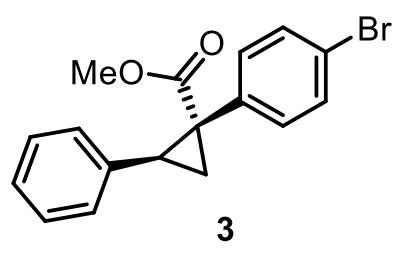

3

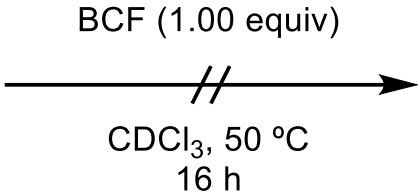

$16 \mathrm{~h}$

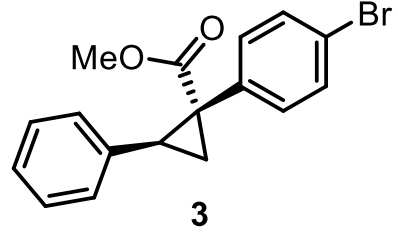

$<5 \%$ change by ${ }^{1} \mathrm{H}$ NMR

Within a $\mathrm{N}_{2}$ filled glove box, cyclopropane carboxylate (azeotroped to dryness from benzene) $\mathbf{3}$ (10 mg, $30 \mu \mathrm{mol}, 92 \%$ purity), tris(pentafluorophenyl)borane (15 mg, $30 \mu \mathrm{mol}$ ), and $\mathrm{CDCl}_{3}$ (300 $\mu \mathrm{L}$ ) were added to a $4 \mathrm{~mL}$ dram vial. The treads of the vial were then lined with Teflon tape, sealed and heated to $50^{\circ} \mathrm{C}$ for $16 \mathrm{~h}$. The vial was then brought outside of the glove box and 100 $\mu \mathrm{L}$ of a stock solution of mesitylene $\left(140 \mu \mathrm{L}\right.$ in $\left.10 \mathrm{~mL} \mathrm{CDCl}_{3}\right)$ were added to the clear homogenous solution. The solution was then diluted with $\mathrm{CDCl}_{3}(400 \mu \mathrm{L})$ and analyzed by ${ }^{1} \mathrm{H}$ NMR. Using the mesitylene resonance at $\delta=2.28 \mathrm{ppm}$ and the methyl ester resonance for 3 at $\delta=3.65 \mathrm{ppm}$, $27.3 \mu \mathrm{mol}$ of $\mathbf{3}(91 \%)$ remain using the equation below:

$$
\frac{\mu \mathrm{mol} \text { (mesitylene) }}{\mu \mathrm{mol}(\mathbf{3})}=\frac{\int(\text { mesitylene }) / 9 H}{\int(\mathbf{3}) / 3 H}
$$

Upon correcting for the initial amount of benzene present in 3 initially $(0.08: 1), 100 \%$ of the cyclopropane product remains unchanged upon heating with $\mathrm{B}\left(\mathrm{C}_{6} \mathrm{~F}_{5}\right)_{3}$.

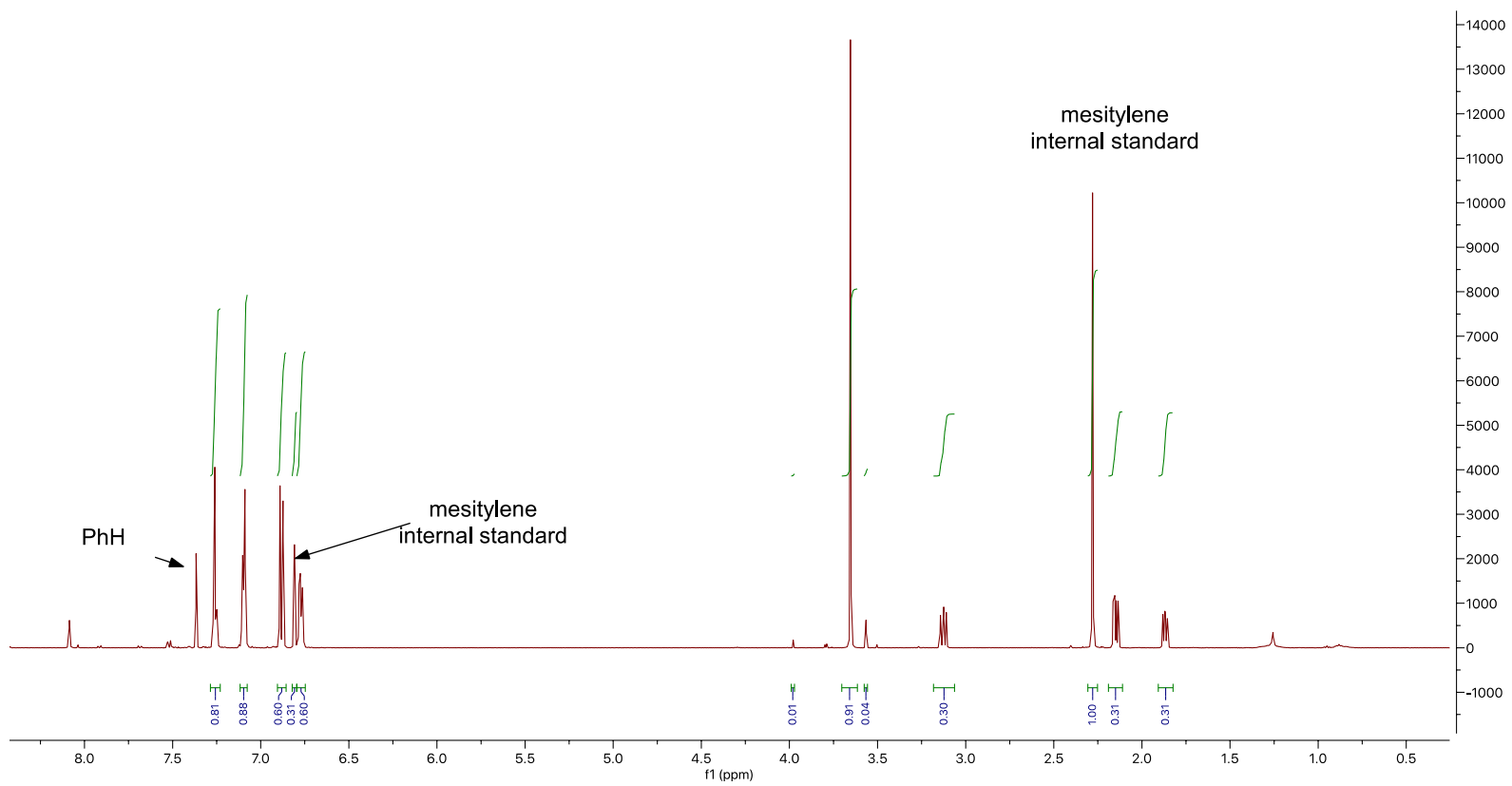


References:

1. Keipour, H; Ollevier, T. Iron-catalyzed carbene insertion reaction of $\alpha$-diazoester into $\mathrm{Si}-$ H bonds. Org. Lett. 2017, 19, 5736-5739.

2. Maier, T. C.; Fu, G. C. Catalytic enantioselective O-H insertion reactions. J. Am. Chem. Soc. 2006, 128, 4594-4595.

3. Peng, C.; Cheng, J.; Wang, J. Palladium-catalyzed cross-coupling of aryl or vinyl iodides with ethyl diazoacetate. J. Am. Chem. Soc. 2007, 129, 8708-8709.

4. Yu, Z.; Li, Y.; Shi, J.; Ma, B.; Liu, L.; Zhang, J. $\left(\mathrm{C}_{6} \mathrm{~F}_{5}\right)_{3} \mathrm{~B}$ catalyzed chemoselective and ortho-selective substitution of phenols with $\alpha$-aryl $\alpha$-diazoesters. Angew. Chem. Int. Ed. 2016, 55, 14807-14811.

5. Stivanin, M. L.; Fernandes, A. G.; da Silva, A. F.; Okada, C. Y.; Jurberg, I. D. Blue lightpromoted $\mathrm{N}-\mathrm{H}$ insertion of carbazoles, pyrazoles, and 1,2,3-triazoles into aryldiazoacetates. Adv. Synth. Cat. 2020, 362, 1106-1111.

6. Fujita, T.; Yamamoto, T.; Morita, Y.; Chen, H.; Shimizu, Y.; Kanai, M. Chemo- and enantioselective $\mathrm{Pd} / \mathrm{B}$ hybrid catalysis for the constructions of acyclic quaternary carbons: migratory allylation of $O$-allyl ester to $\alpha$-C-allyl carboxylic acids. J. Am. Chem. Soc. 2018, 140, 5899-5903.

7. Marcum, J. S.; Cervarich, T. N.; Manan, R. S.; Roberts, C. C.; Meek, S. J. (CDC)Rhodium-catalyzed hydroallylation of vinylarenes and 1,3-dienes with allyltrifluoroborates. ACS Catal. 2019, 9, 5881-5889.

8. Spoehrle, S. S. M.; West, T. H.; Taylor, J. E.; Slawin, A. M. Z.; Smith, A. D. Tandem pallaidum isothiourea relay catalysis: enantioselective synthesis of $\alpha$-amino acid derivatives via allylic amination and [2,3]-sigmatropic rearrangement. J. Am. Chem. Soc. 2017, 139, 11895-11902.

9. Yu, S.; Noble, A.; Bedford, R. B.; Aggarwal, V. K. Methylenespiro[2.3]hexanes via nickel-catalyzed cyclopropanations with [1.1.1]propellane. J. Am. Chem. Soc. 2019, 141, 20325-20334.

10. Yamamoto, T.; Yamakawa, T. Nickel-catalyzed vinylation of aryl chlorides and bromides with vinyl $\mathrm{ZnBr} \cdot \mathrm{MgBrCl}$. J. Org. Chem. 2009, 74, 3603-3605.

11. Denmark, S. E.; Butler, C.R. Vinylation of aryl bromides using an inexpensive vinylpolysiloxane. Org. Lett. 2006, 8, 63-66.

12. Zhang, C.; Wang, Z.; Chen, Q.; Zhang, C.; Gu, Y.; Xiao, J. Generation of the $\mathrm{CF}_{3}$ radical trifluoromethylsulfonium triflate and its trifluoromethylation of styrenes. Chem. Commun. 2011, 47, 6632-6634.

13. Smith, C.R.; RajanBabu, T.V. Low pressure vinylation of aryl and vinyl halides via Heck-Mizoroki reactions using ethylene. Tetrahedron, 2010, 66, 1102-1110.

14. Uthoff, F.; Löwe, J.; Harms, C.; Donsbach, K.; Gröger, H. Chemoenzymatic synthesis of a chiral ozanimod key intermediate starting from naphthalene as cheap petrochemical feedstock. J. Org. Chem. 2019, 84, 4856-4866.

15. Davies, H. M. L.; Cantrell, W. R.; Romines, K. R.; Baum, J. S. Synthesis of furans via rhodium(II) acetate-catalyzed reaction of acetylenes with $\alpha$-diazocarbonyls: ethyl 2methyl-5-phenyl-3-furancarboxaldehyde. Org. Synth. 1992, 70, 422.

16. Green, S. P.; Wheelhouse, K. M.; Payne, A. D.; Hallett, J. P.; Miller. P. W.; Bull, J. A. Thermal stability and explosive hazard assessment of diazo compounds and diazo transfer reagents. Org. Process. Res. Dev. 2020, 1, 67-84. 
17. Pirrung, M. C. Appendix 3: Recipes for TLC stains. The Synthetic Organic Chemist's Companion; Wiley: Hoboken, 2007; p 171.

18. Still, W. C.; Kahn, M.; Mitra, A. Rapid chromatographic technique for preparative separations with moderate resolution. J. Org. Chem. 1978, 43, 2923-2925.

19. Ovalles, S. R.; Hansen, J. H.; Davies, H. M. L. Thermally induced cycloadditions of donor/acceptor carbenes. Org. Lett. 2011, 13, 4284-4287.

20. Chen, L.; Bovee, M.O.; Lemma, B.E.; Keithley, K. S. M.; Pilson, S. L.; Coleman, M. G.; Mack, J. An inexpensive and recyclable silver-foil catalyst for the cyclopropanation of alkenes with diazoacetates under mechanochemical conditions. Angew. Chem. Int. Ed. 2015, 54, 11084-11087.

21. Starmans, W. A. J.; Thijs. L.; Zwanenburg, B. Novel chiral dirhodium catalysts derived from aziridine and azetidine carboxylic acid for intermolecular cyclopropanation reactions with methyl phenyldiazoacetate. Tetrahedron. 1998, 54, 629-636.

22. Chepiga, K. M.; Qin, C.; Alford, J. S.; Chennamadhavuni, S.; Gregg, T. M.; Olson, J. P.; Davies, H. M. L. Guide to enatioselective dirhodium(II)-catalyzed cyclopropanation with aryldiazoacetates. Tetrahedron. 2013, 69, 5765-5771.

23. Collins, L. R., Auris, S., Goddard, R., Fürstner, A. Chiral heterobimetallic bismuthrhodium paddlewheel catalysts: a conceptually new approach to asymmetric cyclopropanation. Angew. Chem. Int. Ed. 2019, 58, 3557-3561.

24. Jurberg, I. D.; Davies, H. M. L. Blue light-promoted photolysis of aryldiazoacetates. Chem. Sci. 2018, 9, 5112-5118.

25. Wei, B., Sharland, J. C., Lin, P., Wilkerson-Hill, S. M., Fullilove, F. A., McKinnon, S., Blackmond, D. G., Davies, H. M. L. In situ kinetic studies of Rh (II)-catalyzed asymmetric cyclopropanation with low catalyst loadings. ACS Catal. 2020, 10, 11611170. 
${ }^{1} \mathrm{H}$ and ${ }^{13} \mathrm{C}$ NMR Spectra 
ㄴํํ요소

กヘก๋

ل।

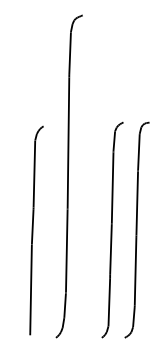

m

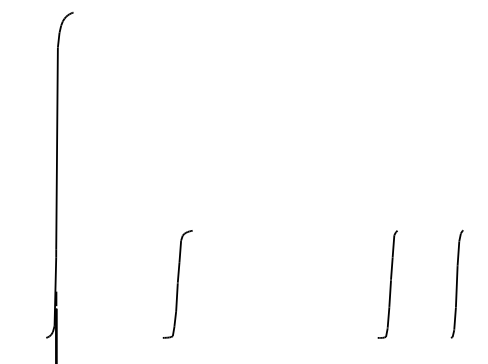

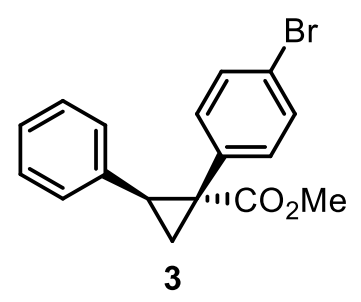

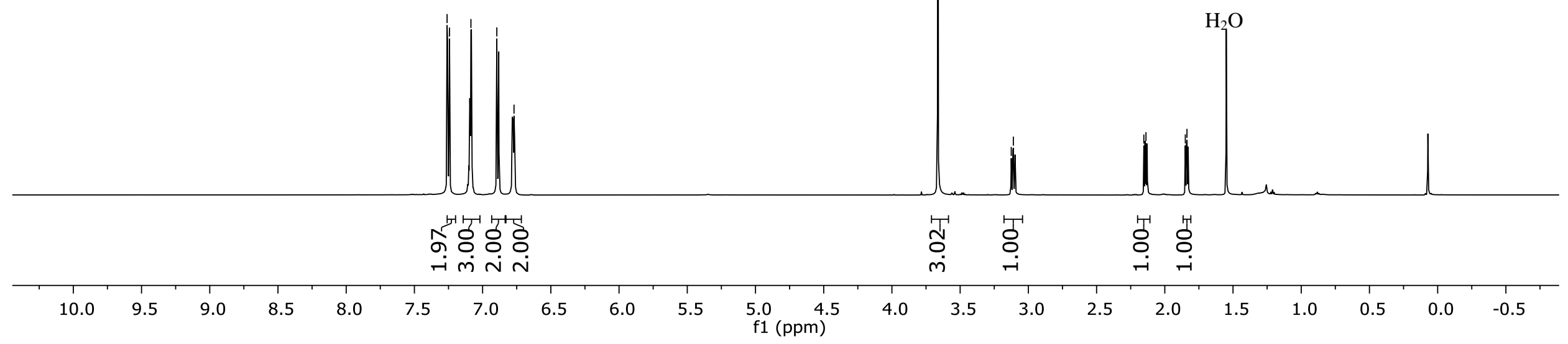

${ }^{1} \mathrm{H} \mathrm{NMR}\left(\mathrm{CDCl}_{3}, 600 \mathrm{MHz}\right)$ 

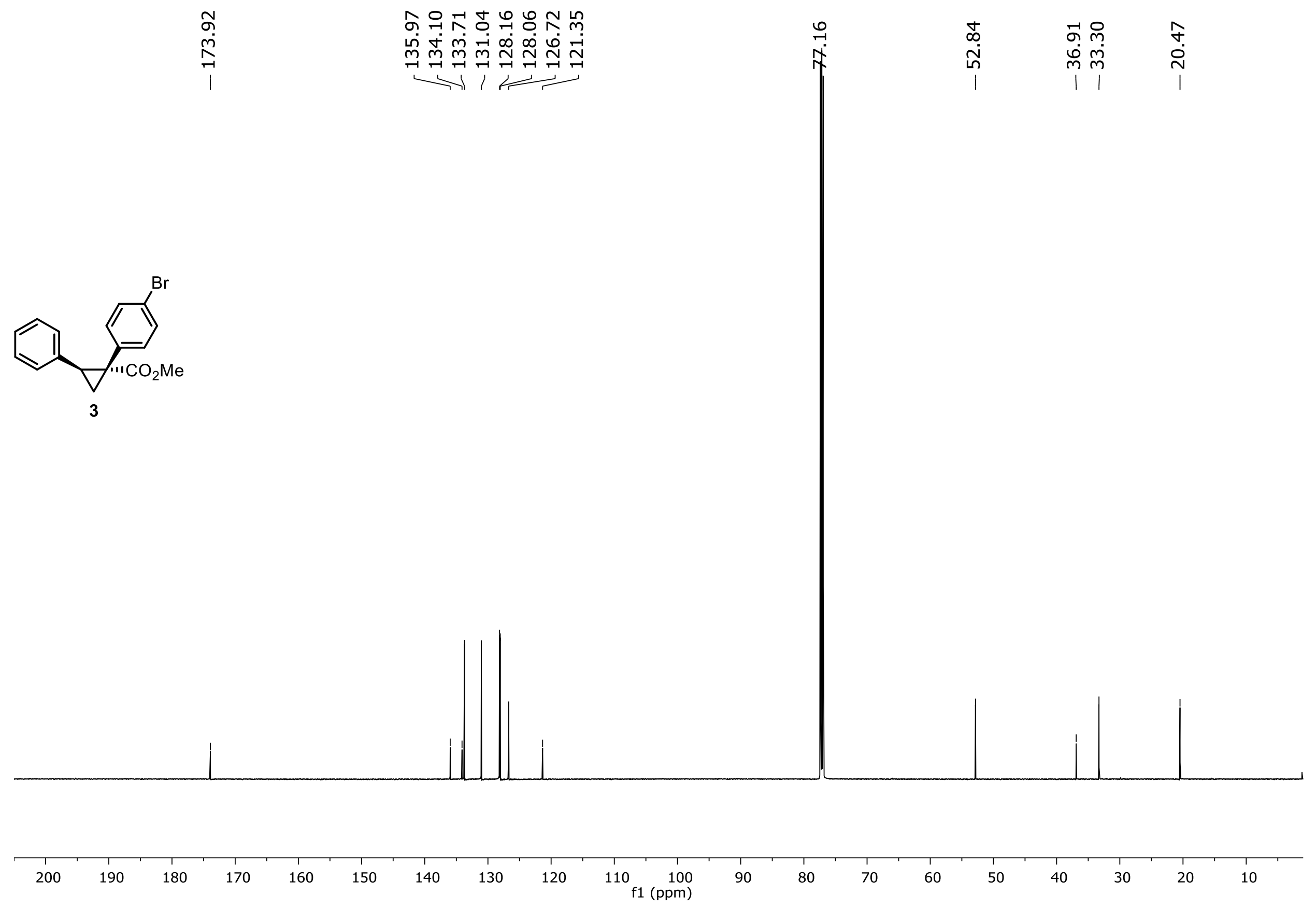

${ }^{1} \mathrm{H} \mathrm{NMR}\left(\mathrm{CDCl}_{3}, 151 \mathrm{MHz}\right)$ 


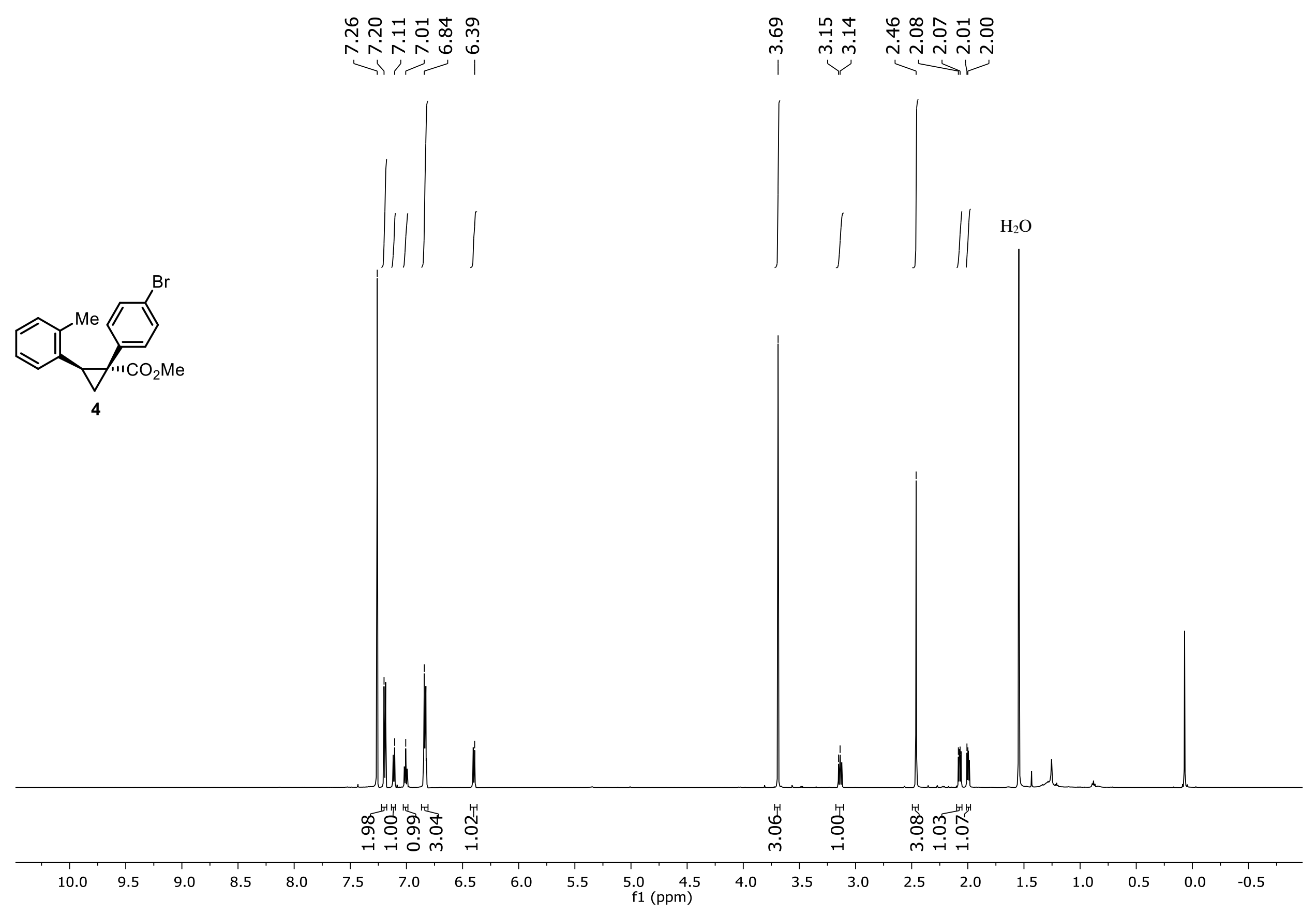

${ }^{1} \mathrm{H} \mathrm{NMR}\left(\mathrm{CDCl}_{3}, 600 \mathrm{MHz}\right)$ 


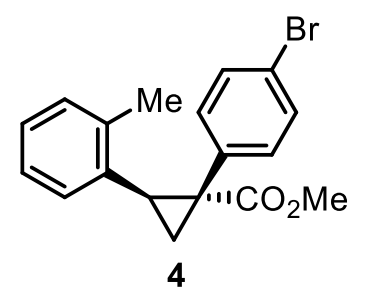

बू ले

लिं ले

공

유

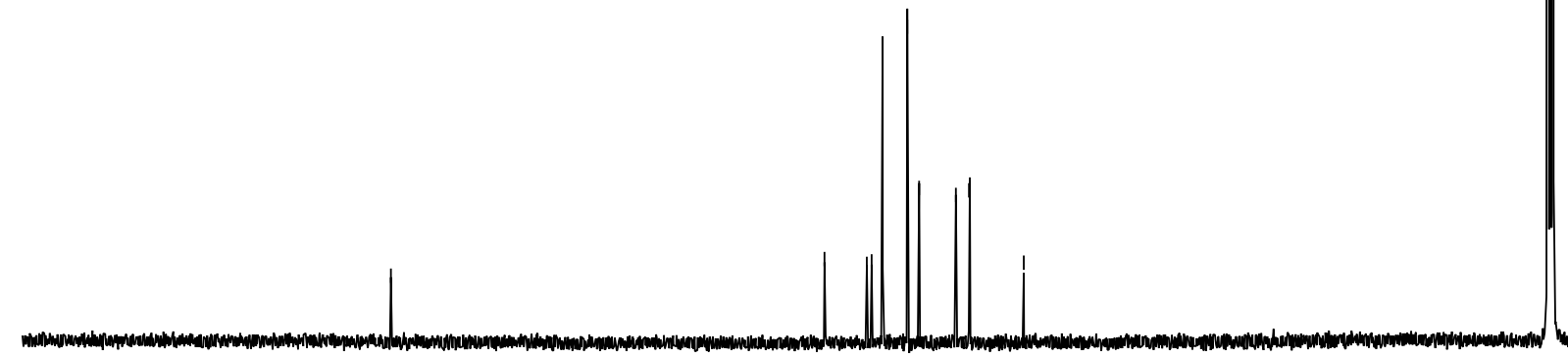




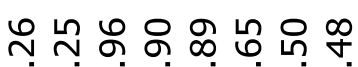

N

$\longrightarrow$
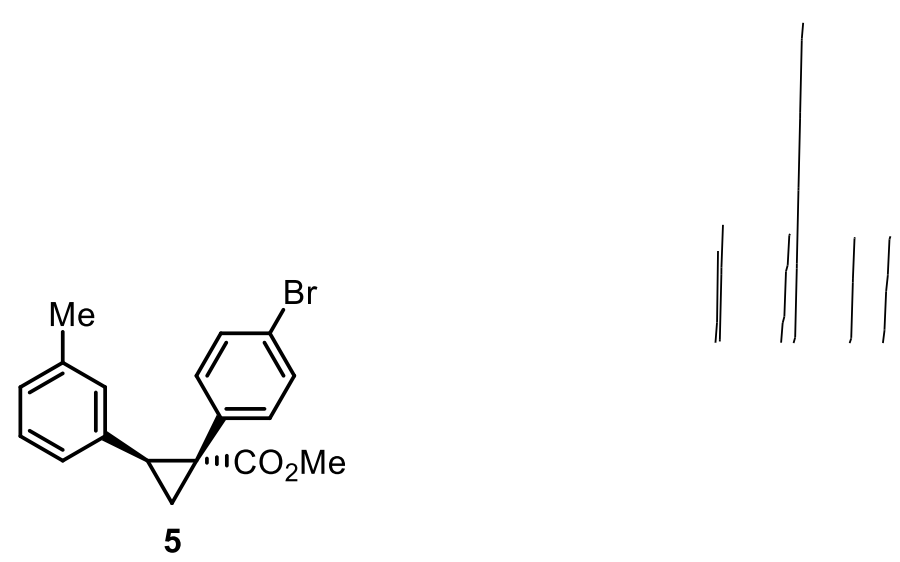

ஜำ

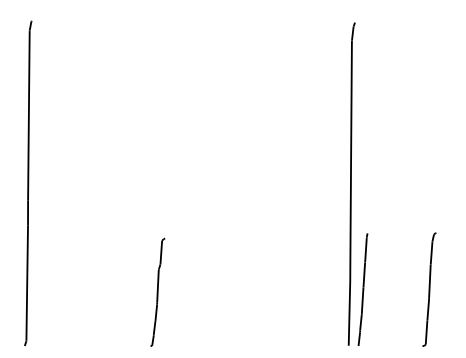

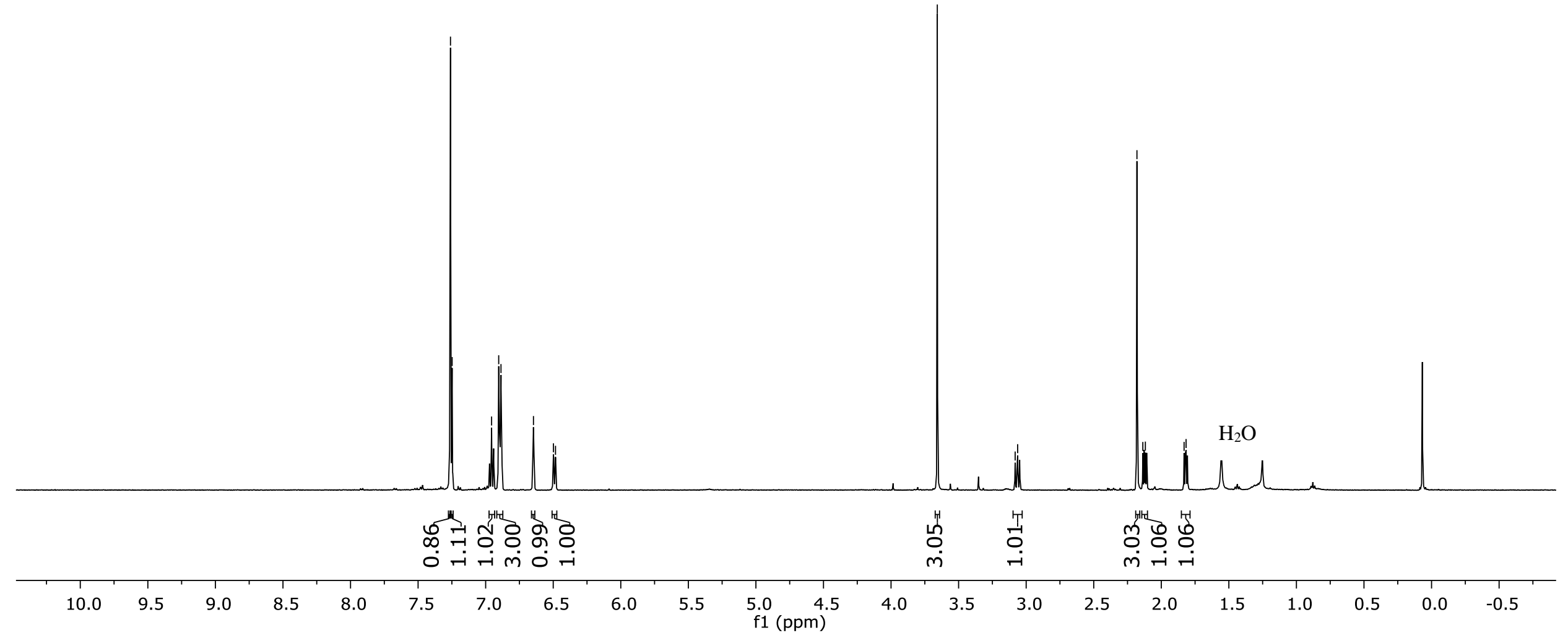

${ }^{1} \mathrm{H} \mathrm{NMR}\left(\mathrm{CDCl}_{3}, 500 \mathrm{MHz}\right)$ 


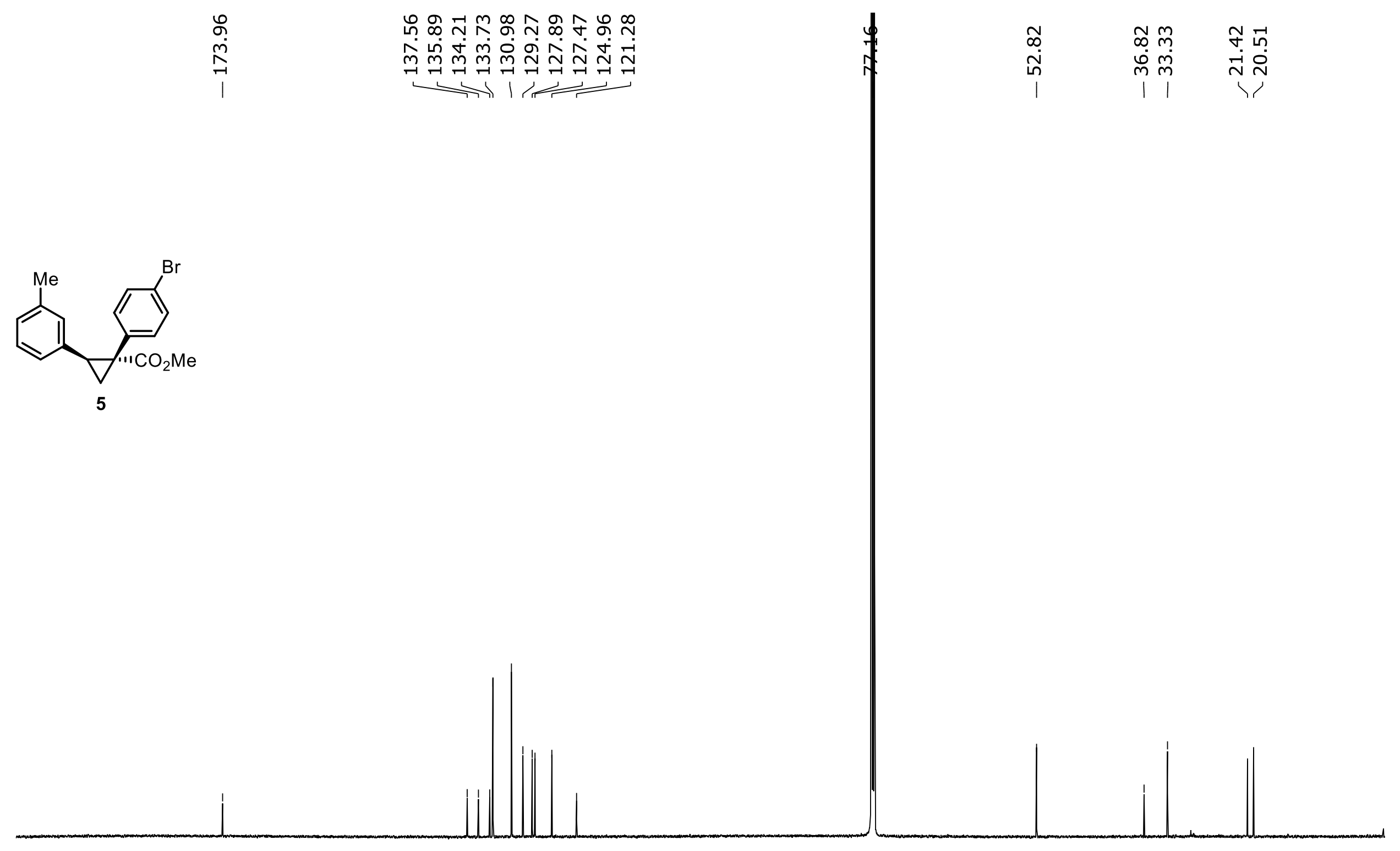

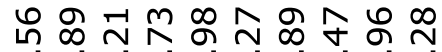

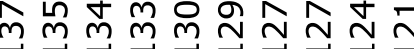

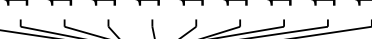

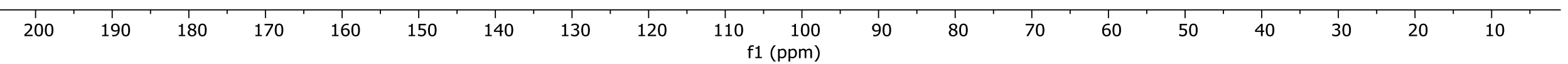

${ }^{1} \mathrm{H} \mathrm{NMR}\left(\mathrm{CDCl}_{3}, 151 \mathrm{MHz}\right)$ 


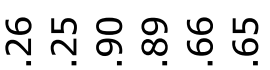

تَ

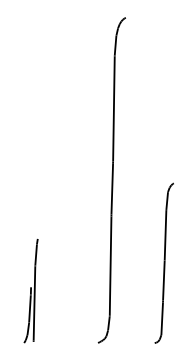

ம0

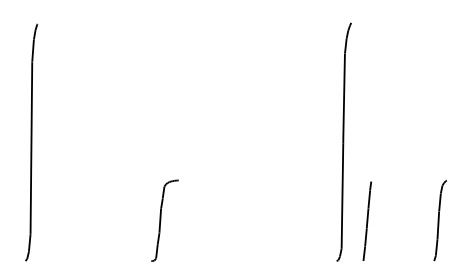

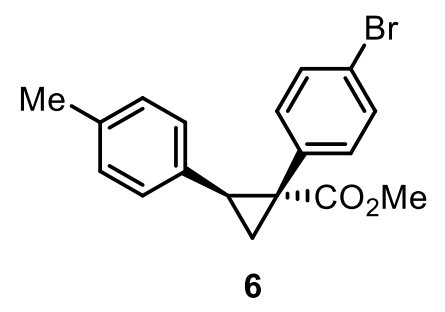
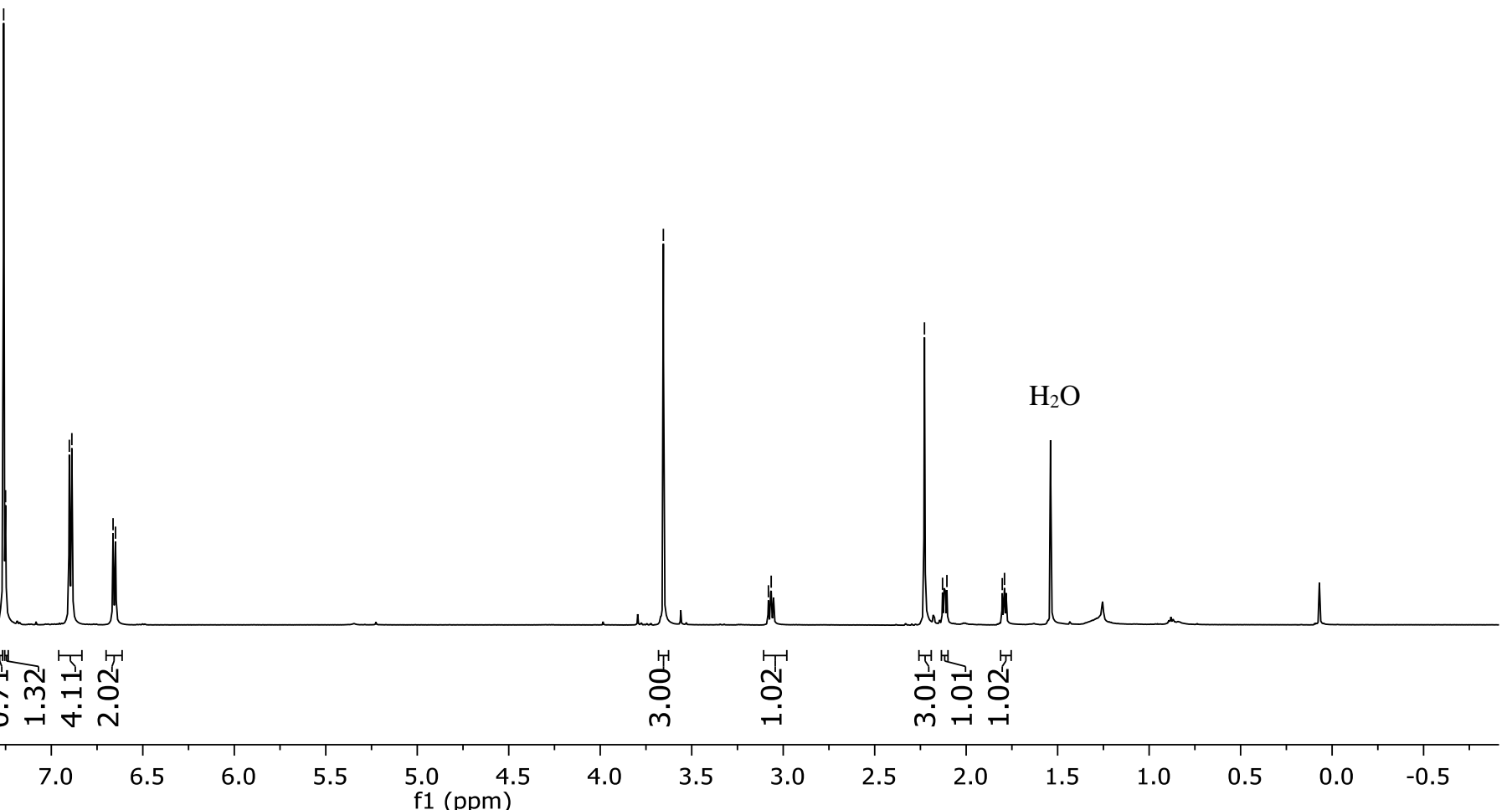

${ }^{1} \mathrm{H} \mathrm{NMR}\left(\mathrm{CDCl}_{3}, 500 \mathrm{MHz}\right)$ 

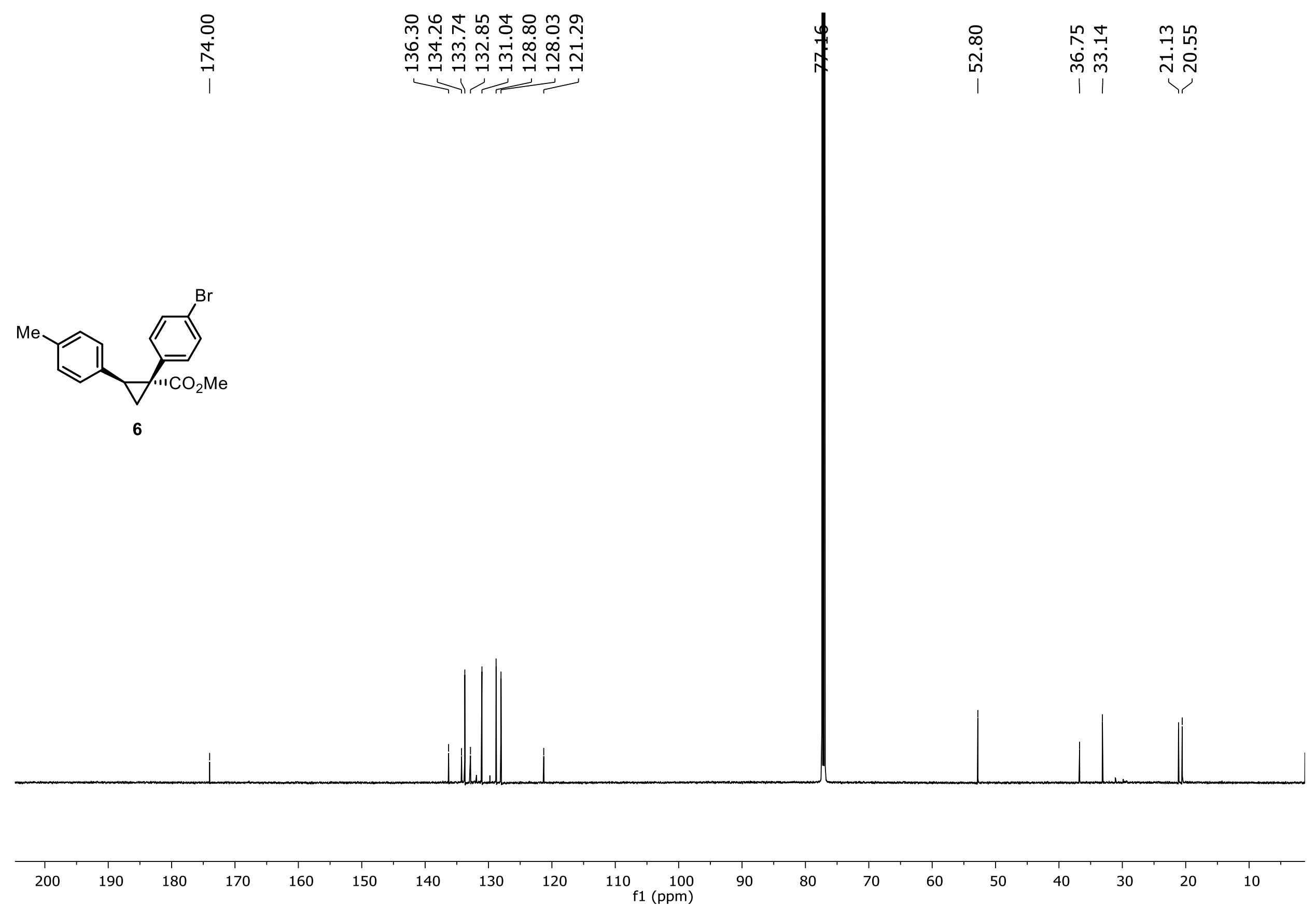

${ }^{13} \mathrm{C} \mathrm{NMR}\left(\mathrm{CDCl}_{3}, 151 \mathrm{MHz}\right)$ 


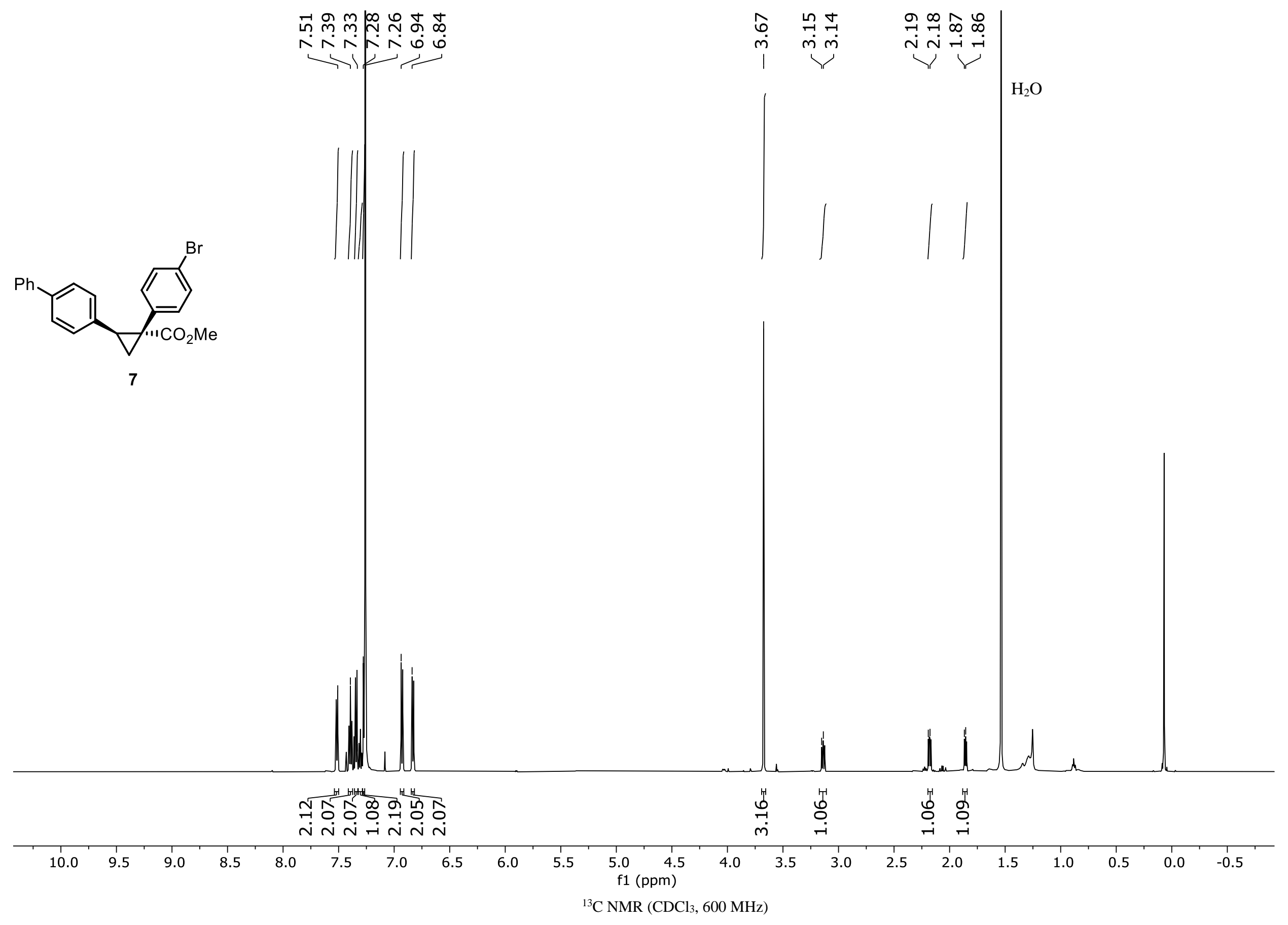


L
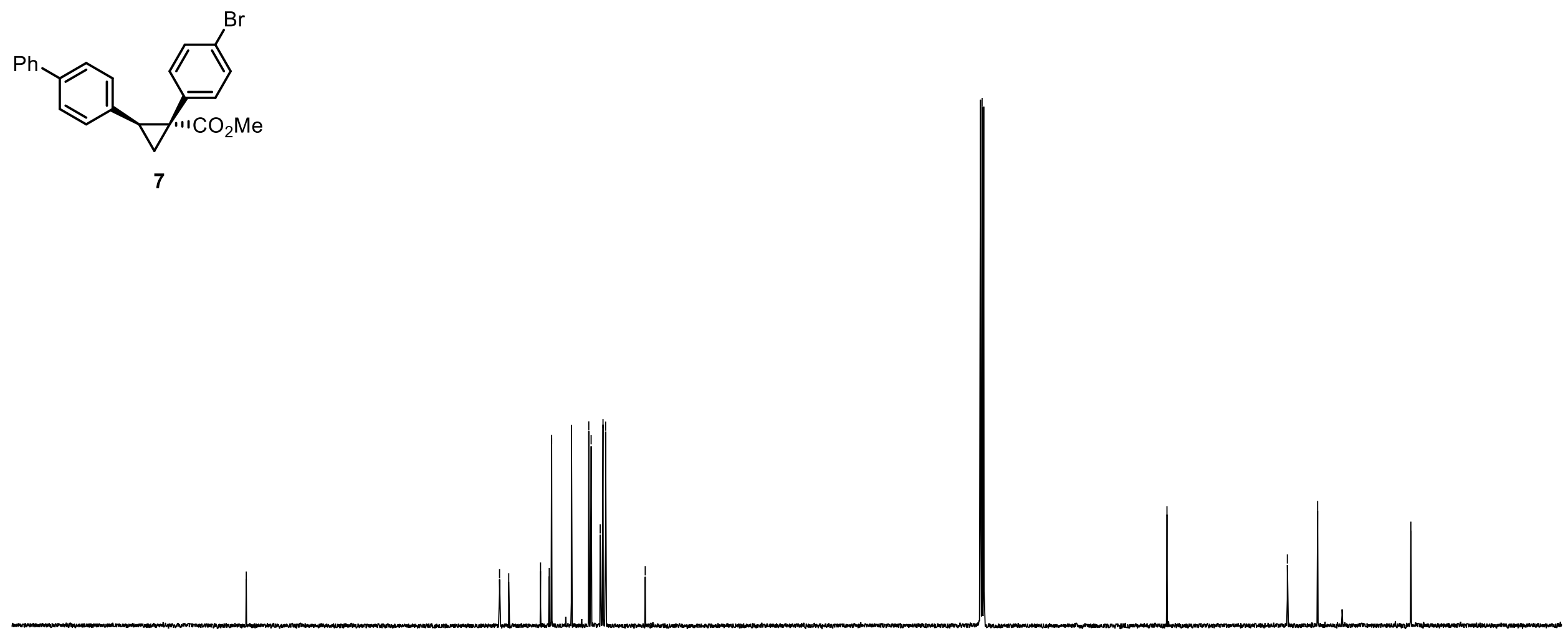

${ }^{13} \mathrm{C} \mathrm{NMR}\left(\mathrm{CDCl}_{3}, 151 \mathrm{MHz}\right)$ 


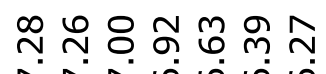

గi,

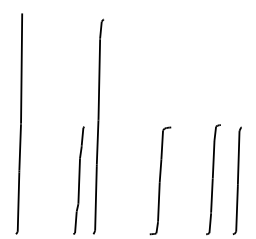

웅 옹

mं mं

mi m

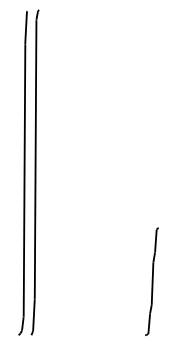

$\underset{\sim}{\stackrel{m}{\sim}} \underset{\infty}{\infty}$

N N

r
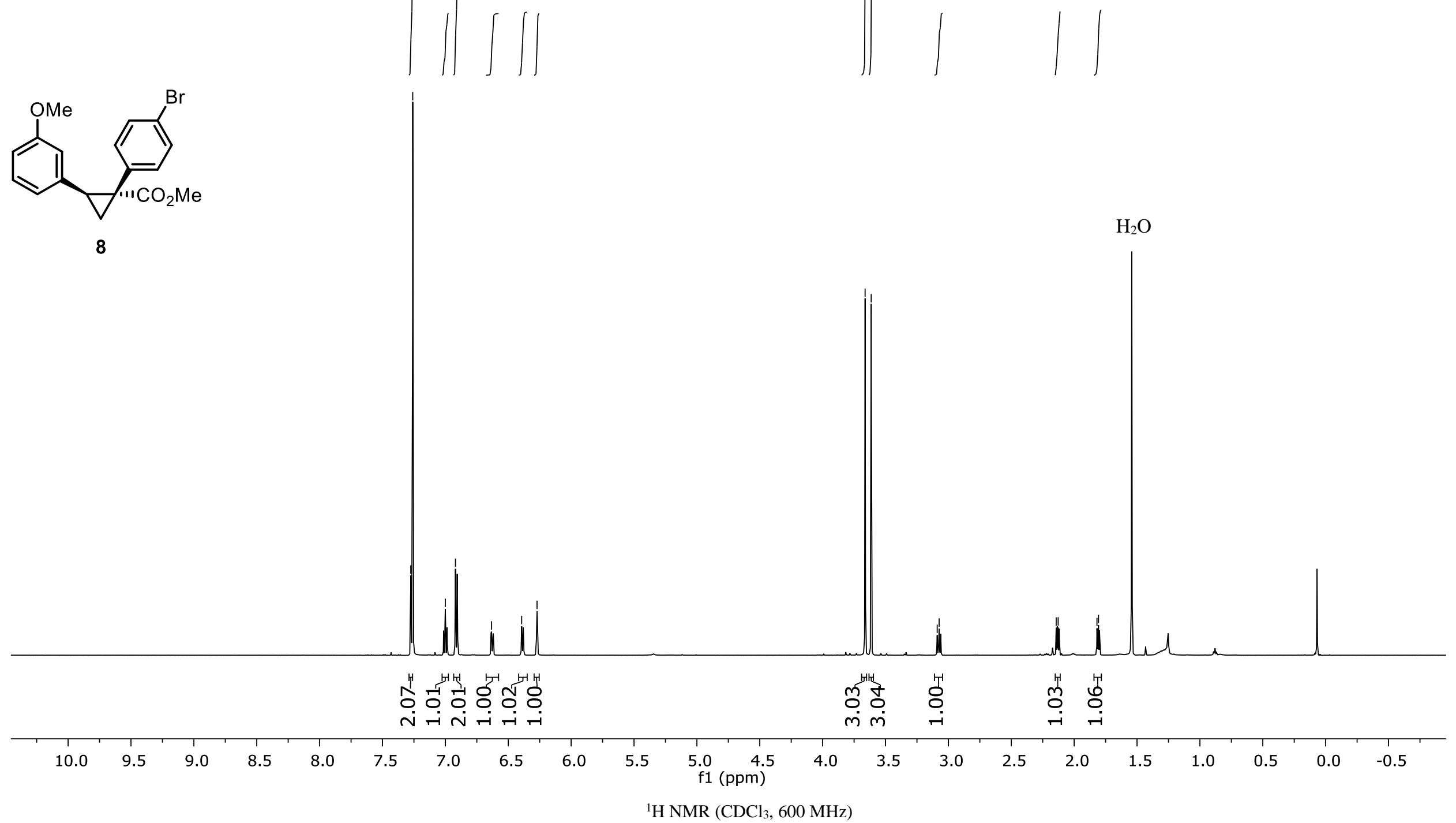
오

เก่

สุกิ रा। ए।
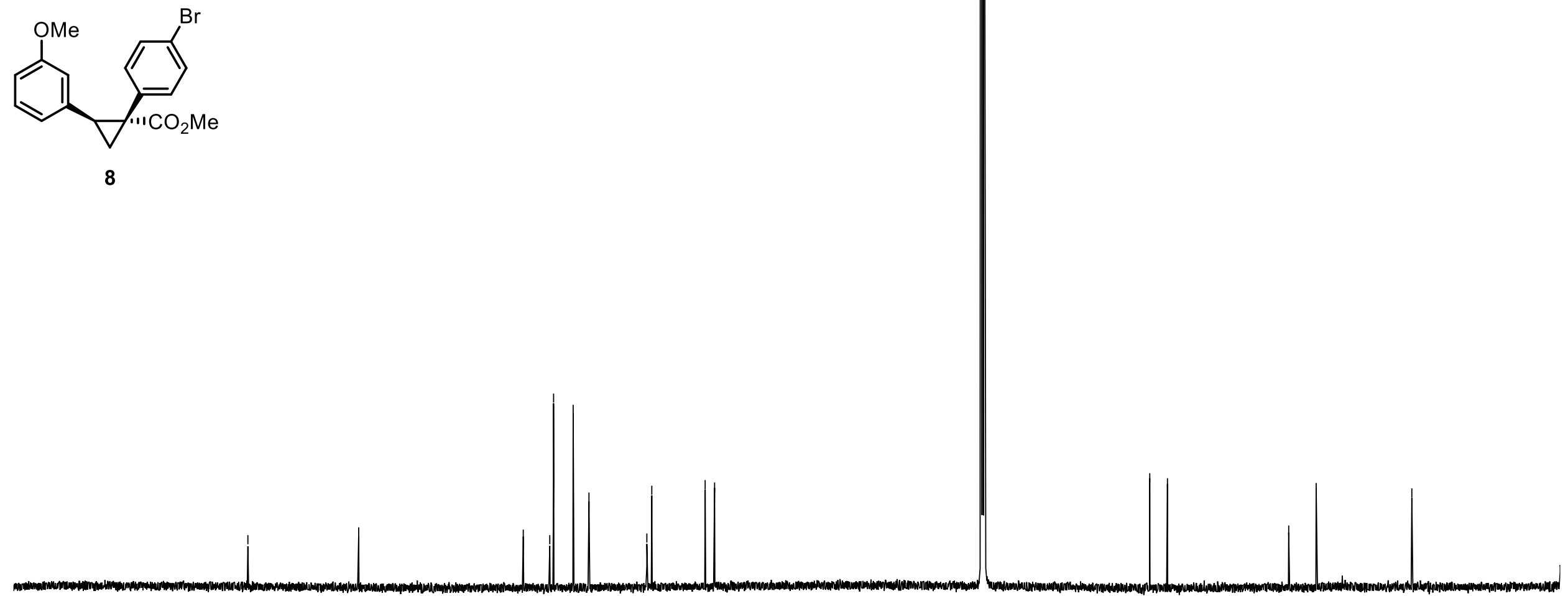

${ }^{13} \mathrm{C} \mathrm{NMR}\left(\mathrm{CDCl}_{3}, 151 \mathrm{MHz}\right)$ 


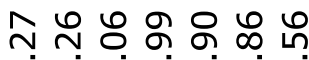

N N

$+11$

m

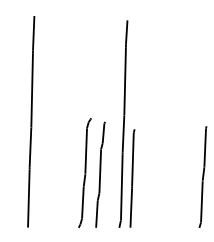

111

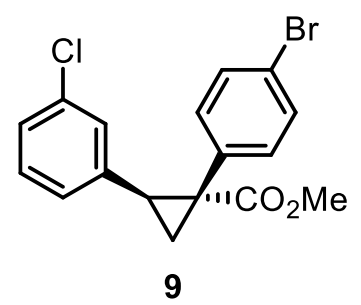

$\mathrm{H}_{2} \mathrm{O}$

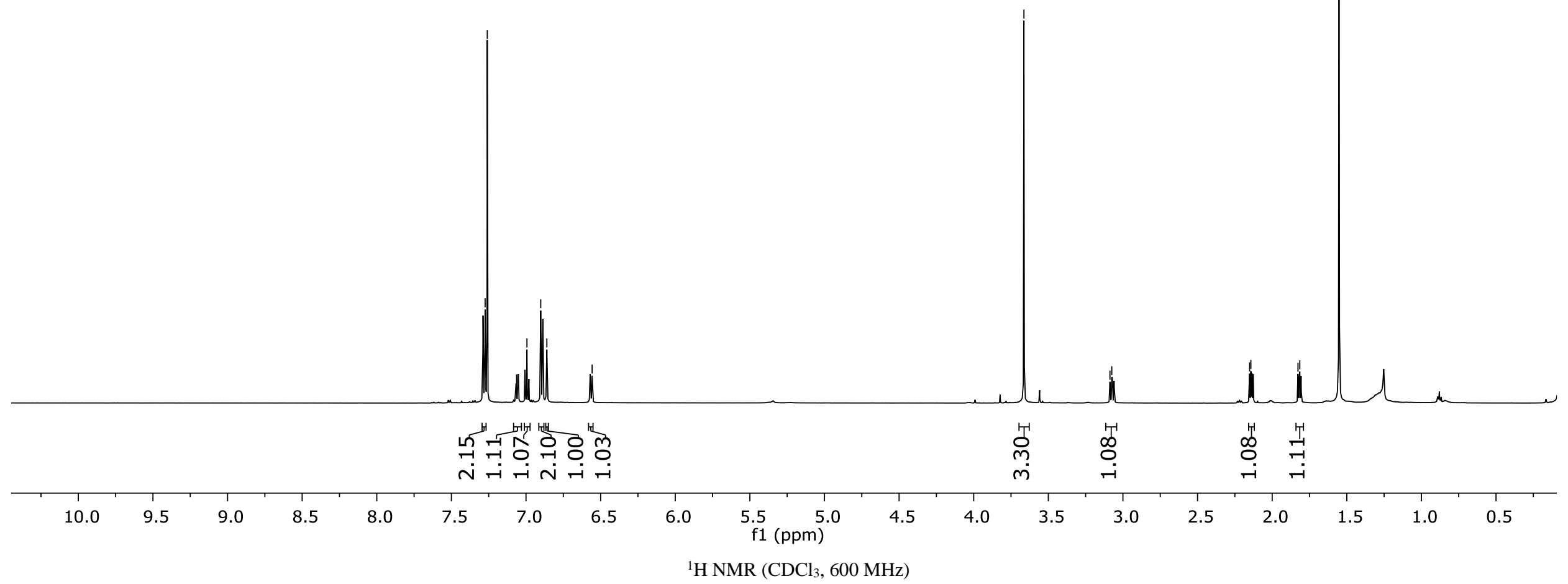



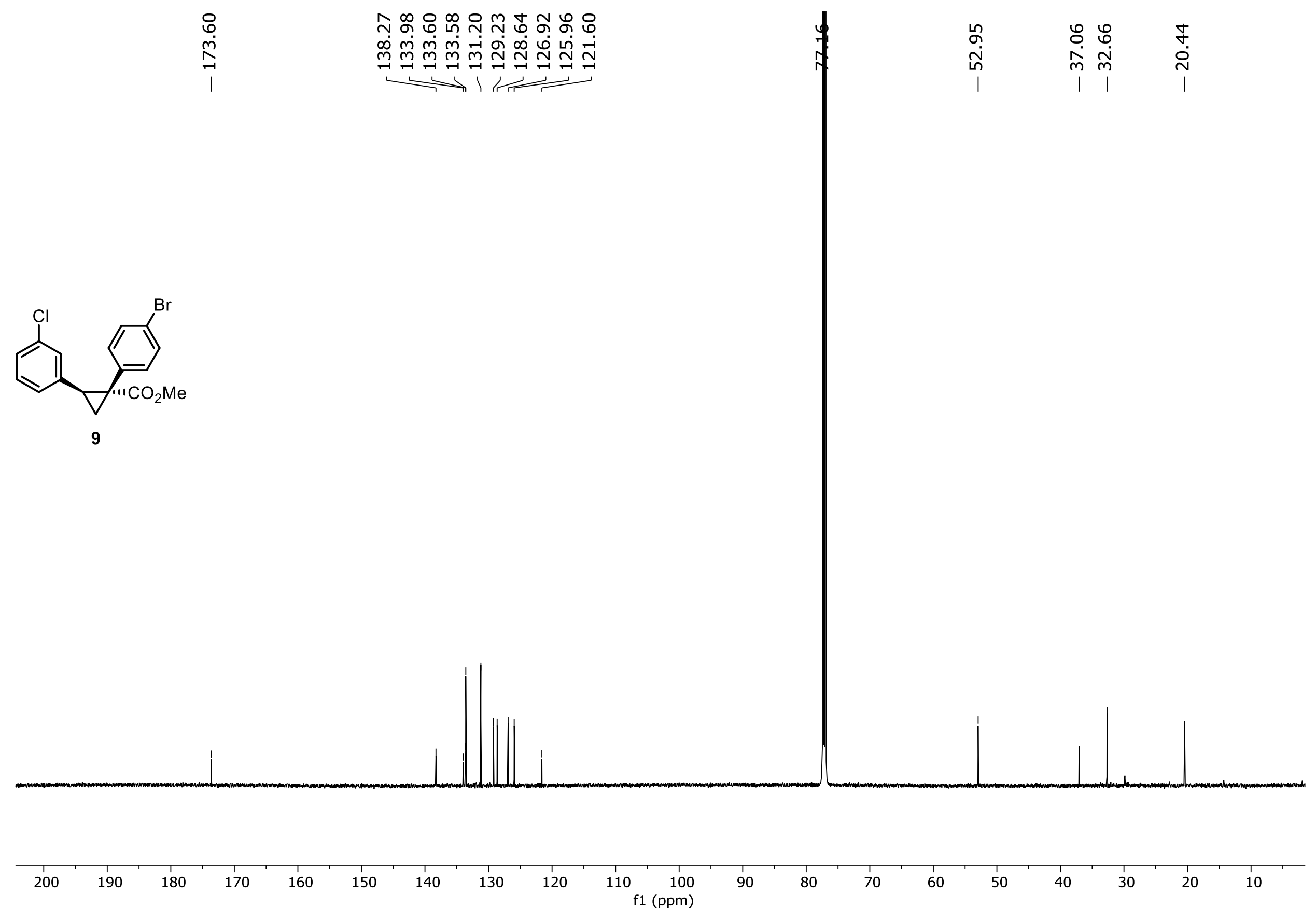

${ }^{13} \mathrm{C} \mathrm{NMR}\left(\mathrm{CDCl}_{3}, 151 \mathrm{MHz}\right)$ 


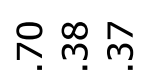

mं mं

$1>$

궁 요 के

N $N$ -

4
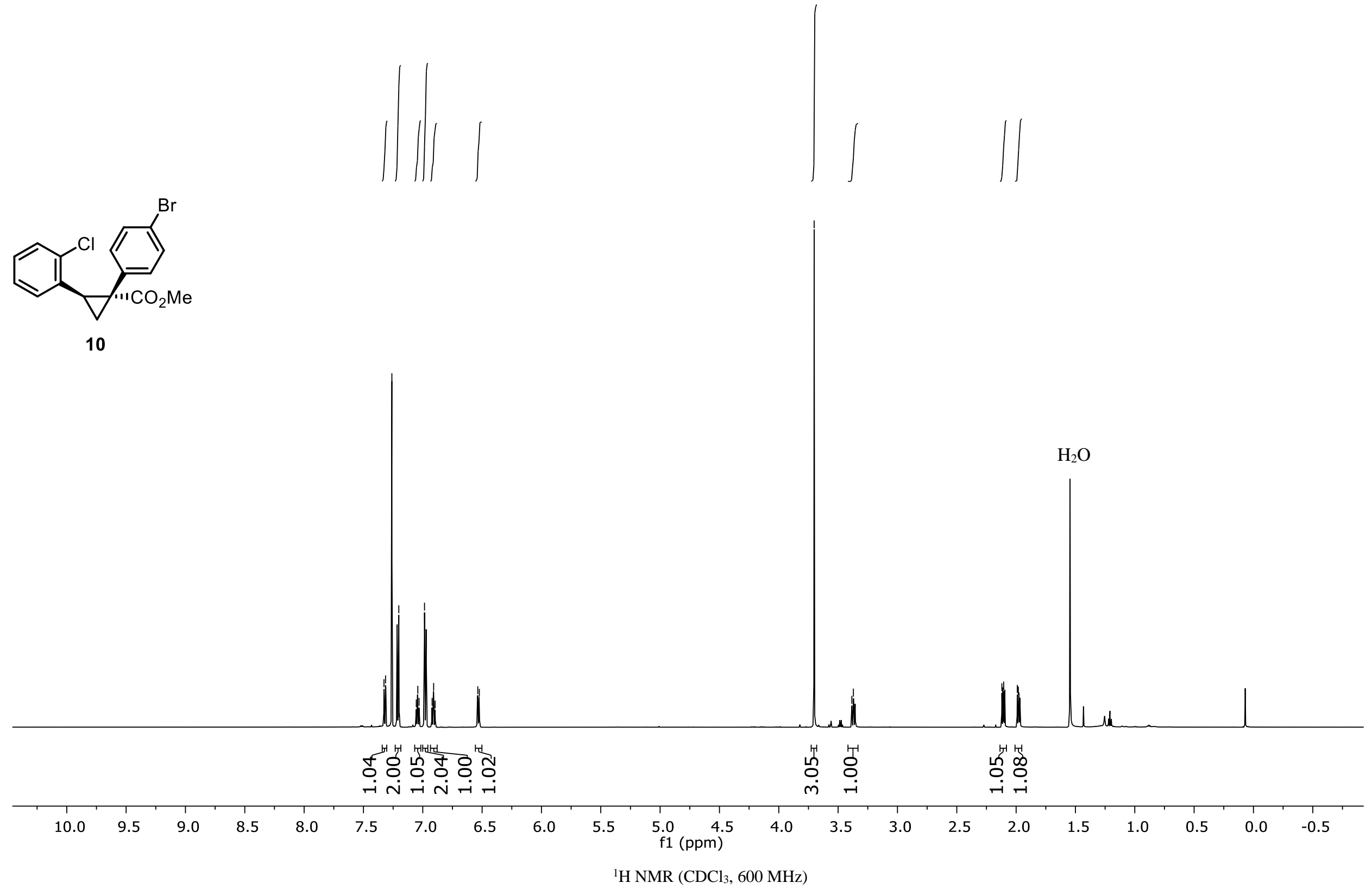


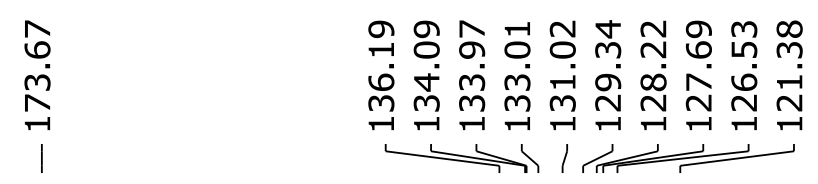

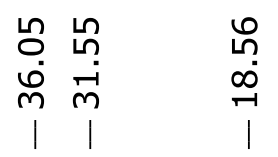
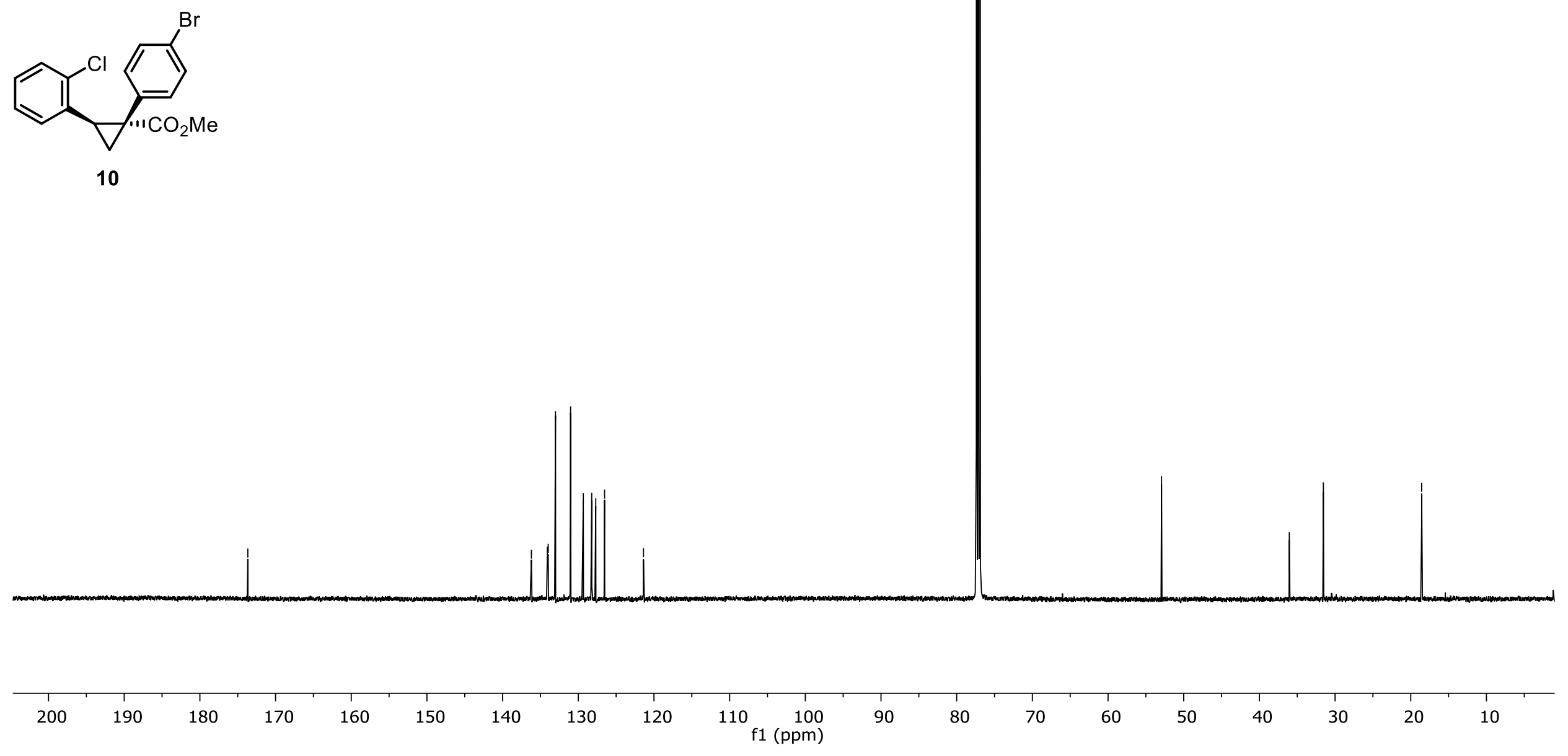

${ }^{13} \mathrm{C}$ NMR (CDCl $\left.3,151 \mathrm{MHz}\right)$ 


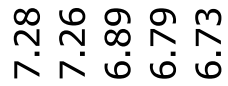

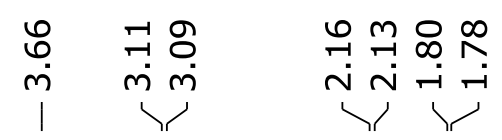
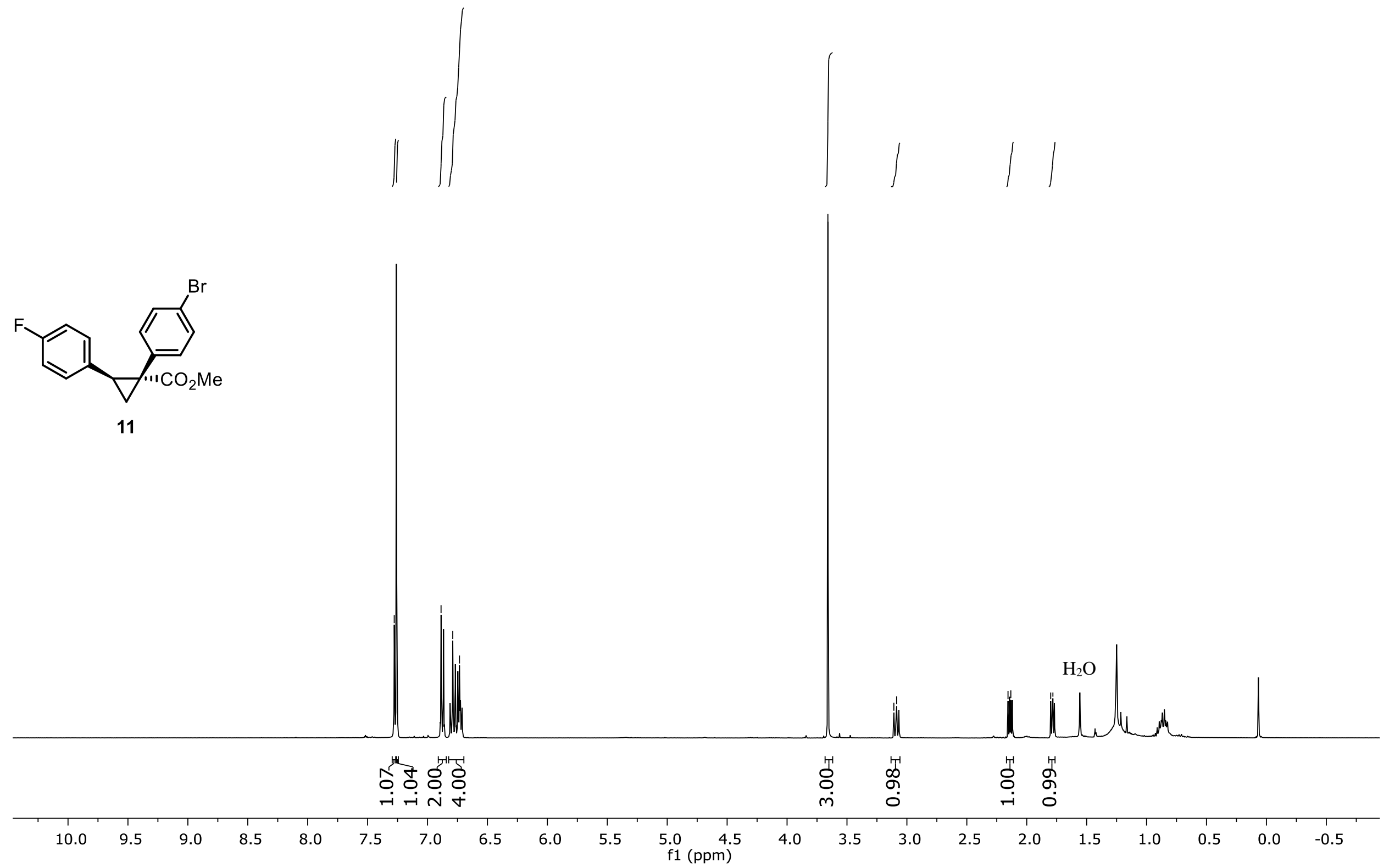

${ }^{1} \mathrm{H} \mathrm{NMR}\left(\mathrm{CDCl}_{3}, 500 \mathrm{MHz}\right)$ 

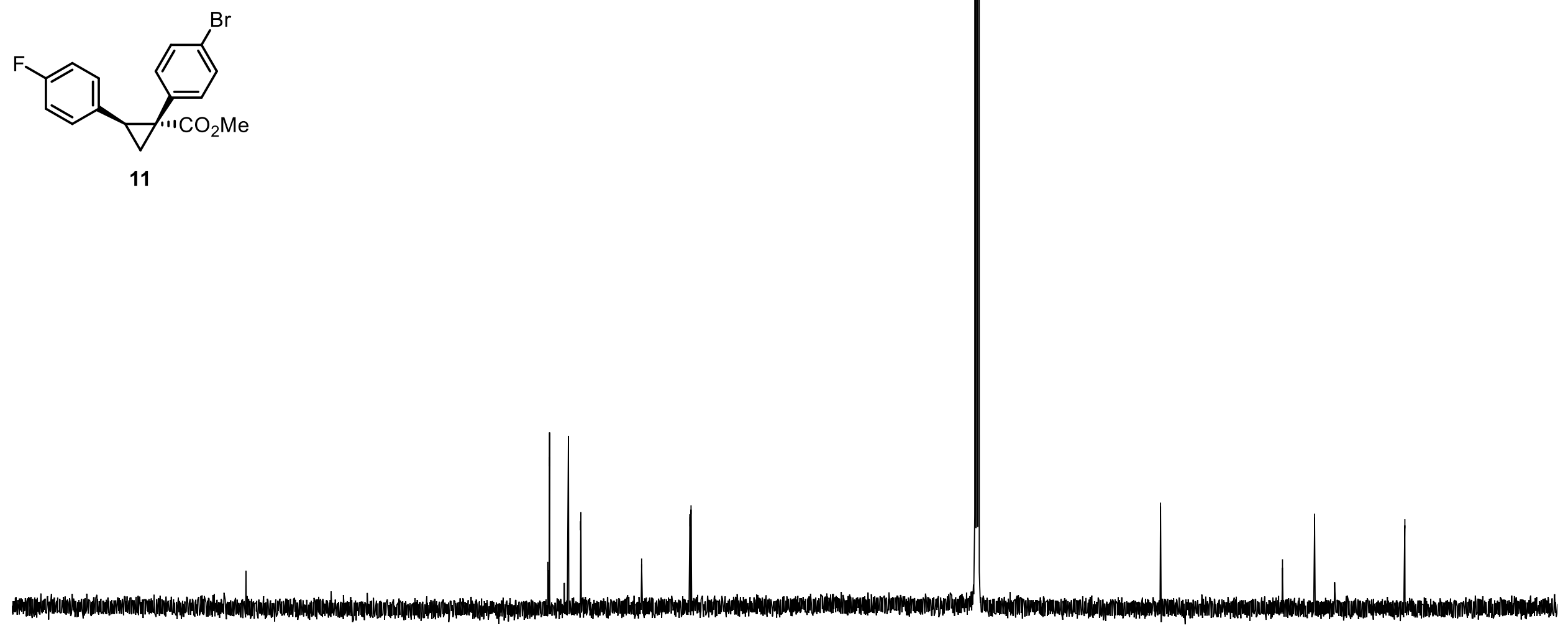

${ }^{13} \mathrm{C} \mathrm{NMR}\left(\mathrm{CDCl}_{3}, 151 \mathrm{MHz}\right)$ 


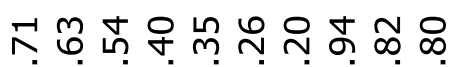

ヘNヘNヘN

요요 m

लं लि

ํํำ

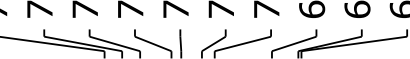

$m m m$

$\underbrace{N}$
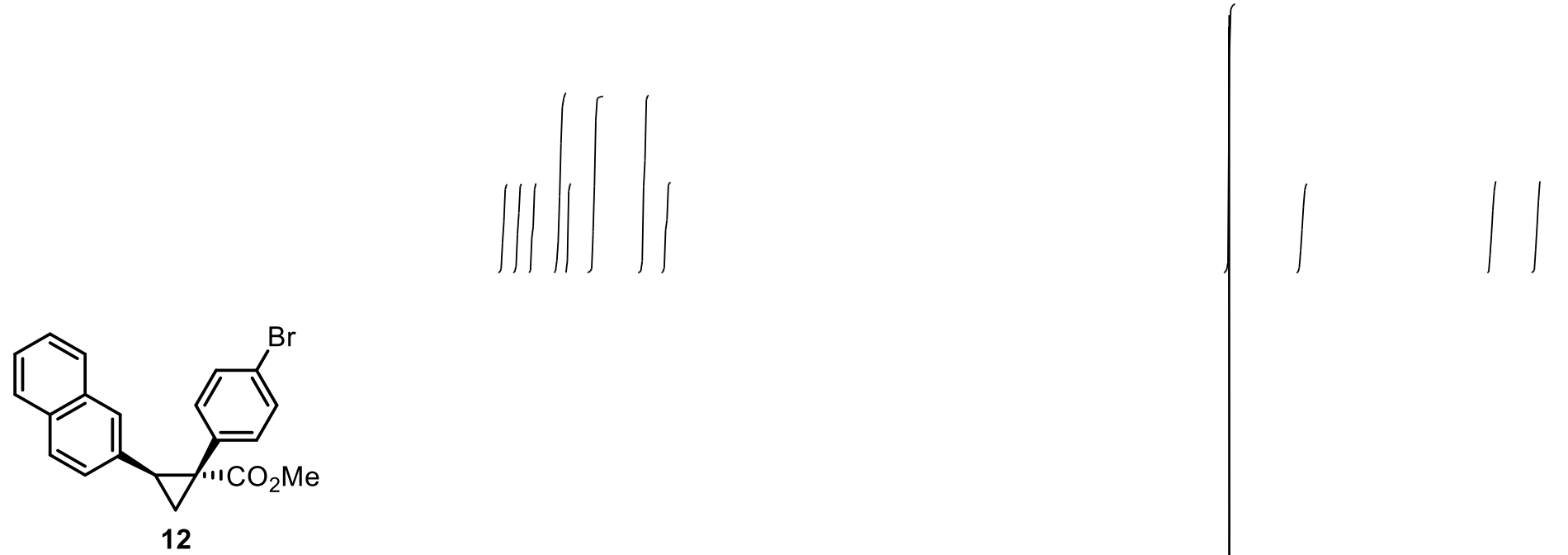

$\mathrm{H}_{2} \mathrm{O}$

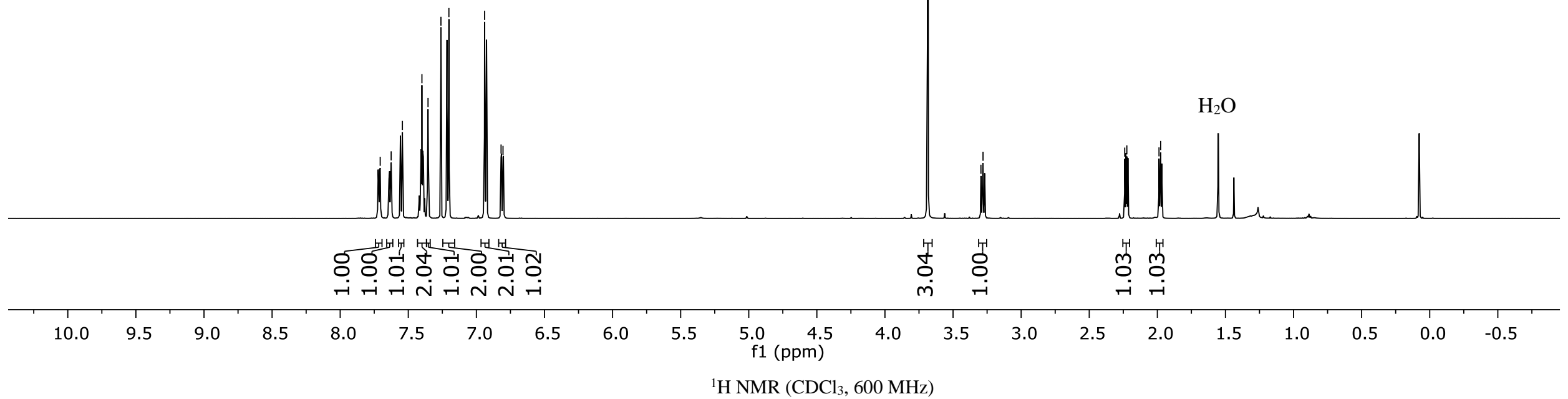



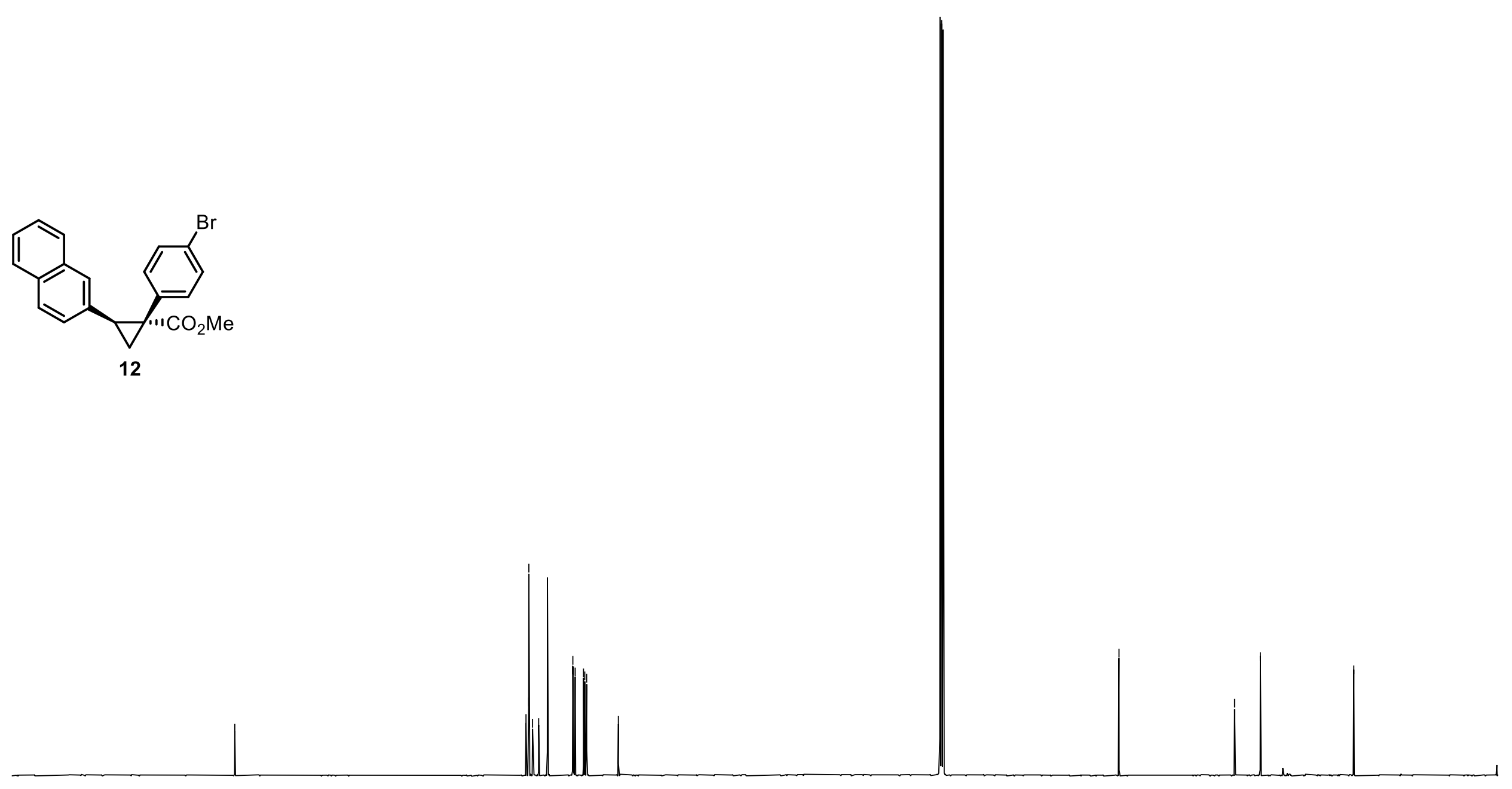

${ }^{1} \mathrm{H} \mathrm{NMR}\left(\mathrm{CDCl}_{3}, 151 \mathrm{MHz}\right)$ 


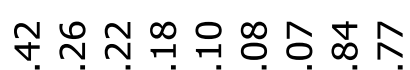

NヘNヘNヘN

$\stackrel{\longrightarrow}{\longrightarrow}$

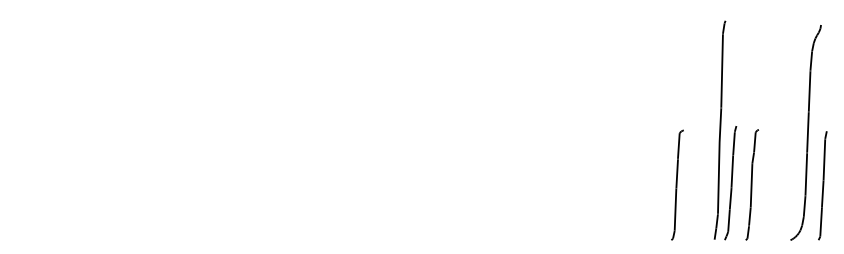

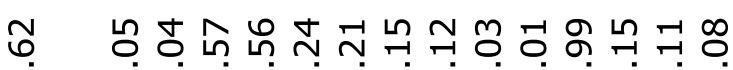

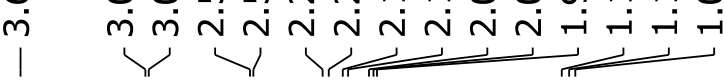
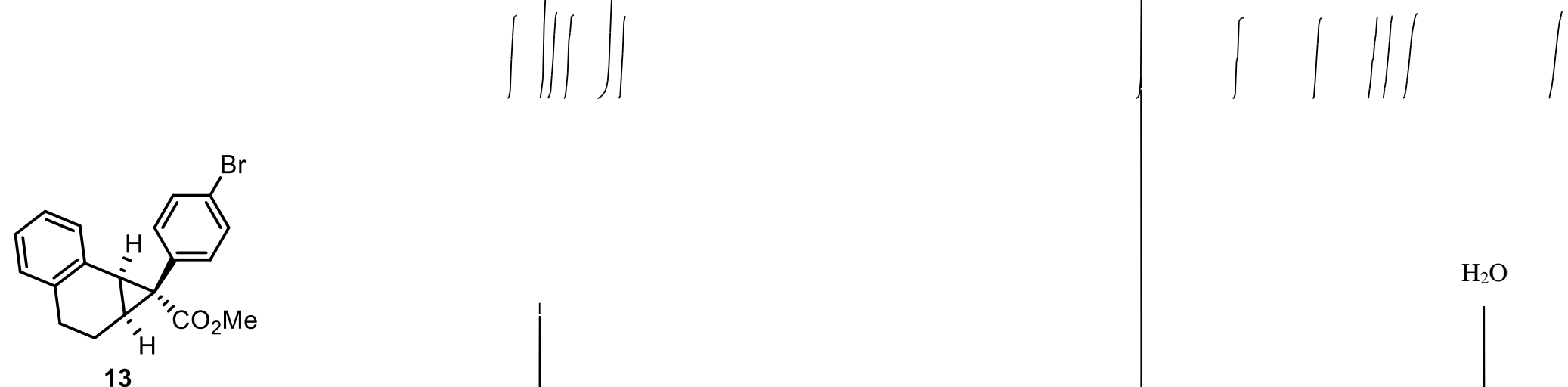

$\mathrm{H}_{2} \mathrm{O}$

13
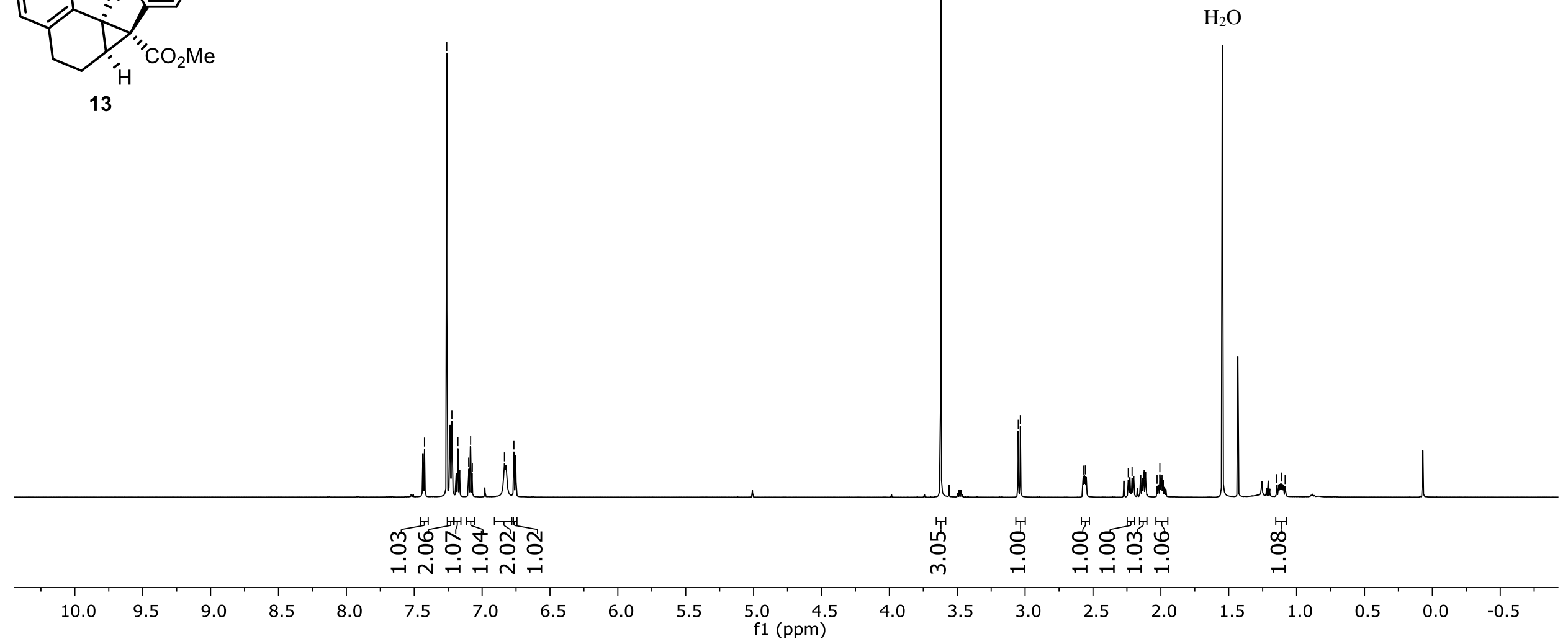

${ }^{1} \mathrm{H} \mathrm{NMR}\left(\mathrm{CDCl}_{3}, 600 \mathrm{MHz}\right)$ 


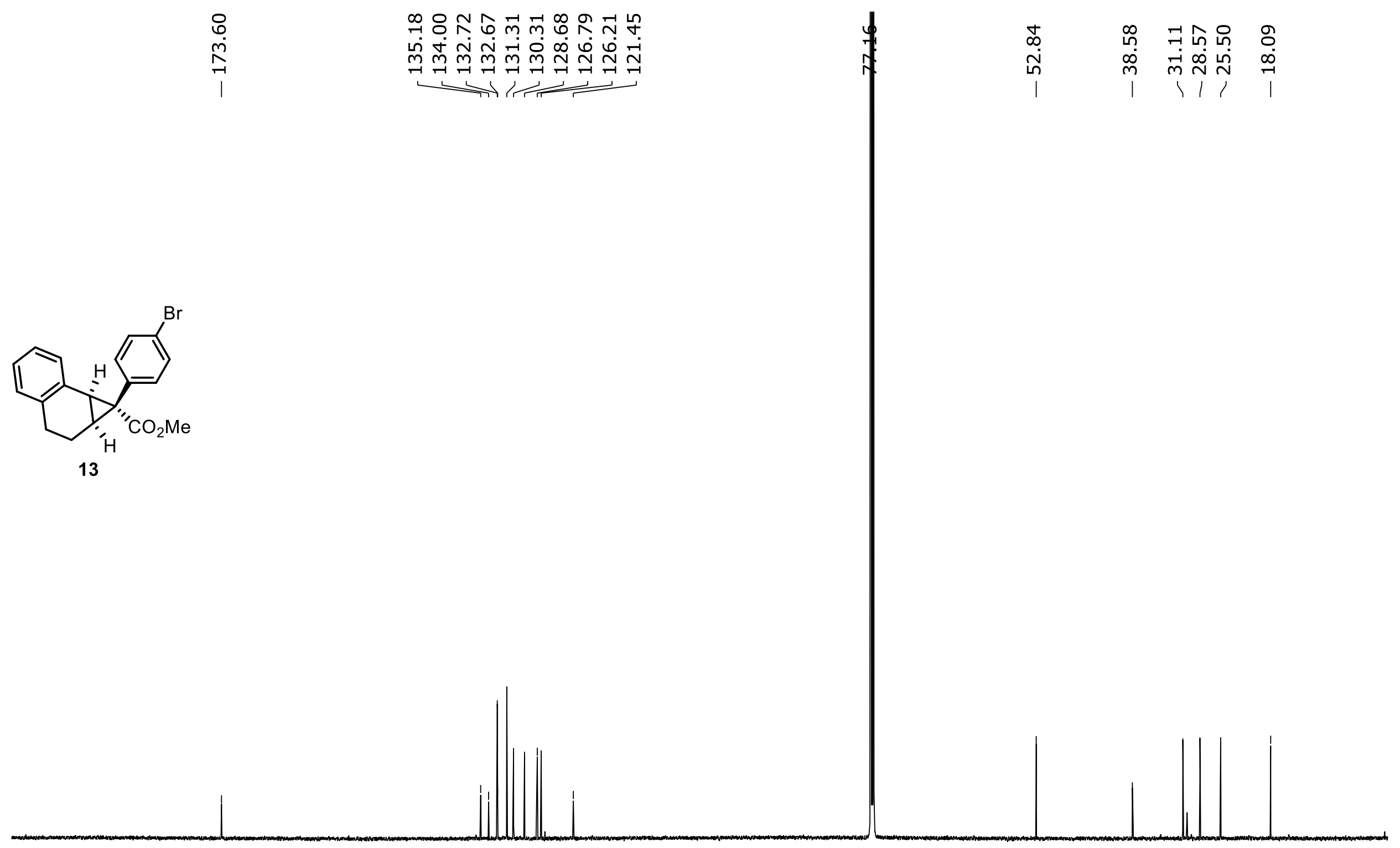

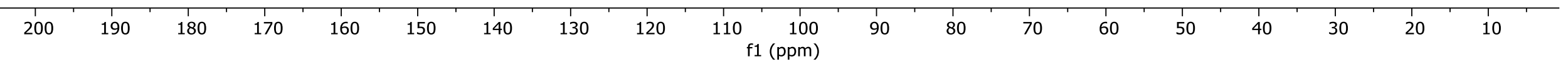

${ }^{13} \mathrm{C} \mathrm{NMR}\left(\mathrm{CDCl}_{3}, 151 \mathrm{MHz}\right)$ 


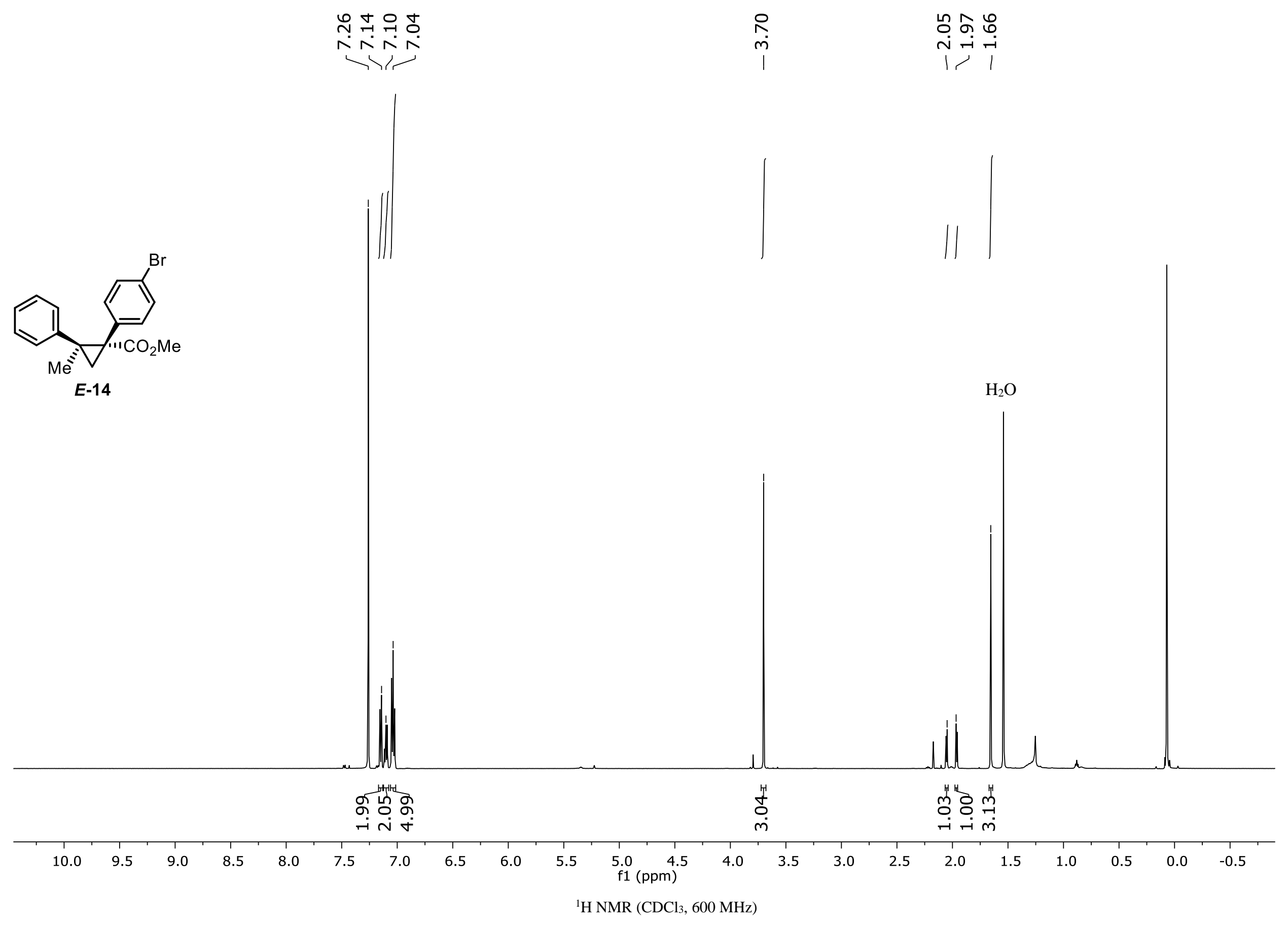




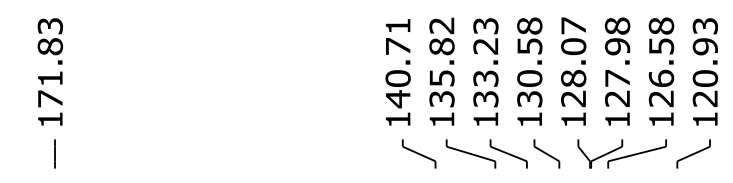

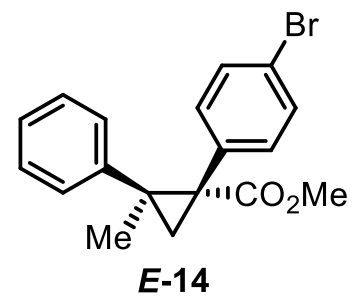

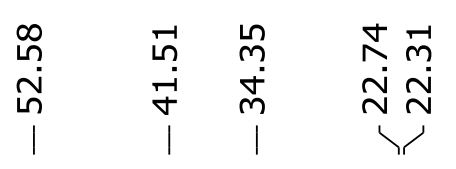

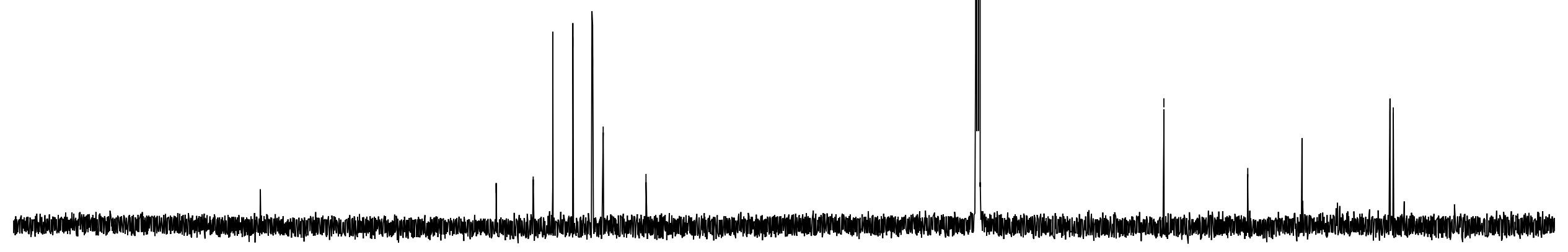

${ }^{1} \mathrm{H} \mathrm{NMR}\left(\mathrm{CDCl}_{3}, 151 \mathrm{MHz}\right)$ 


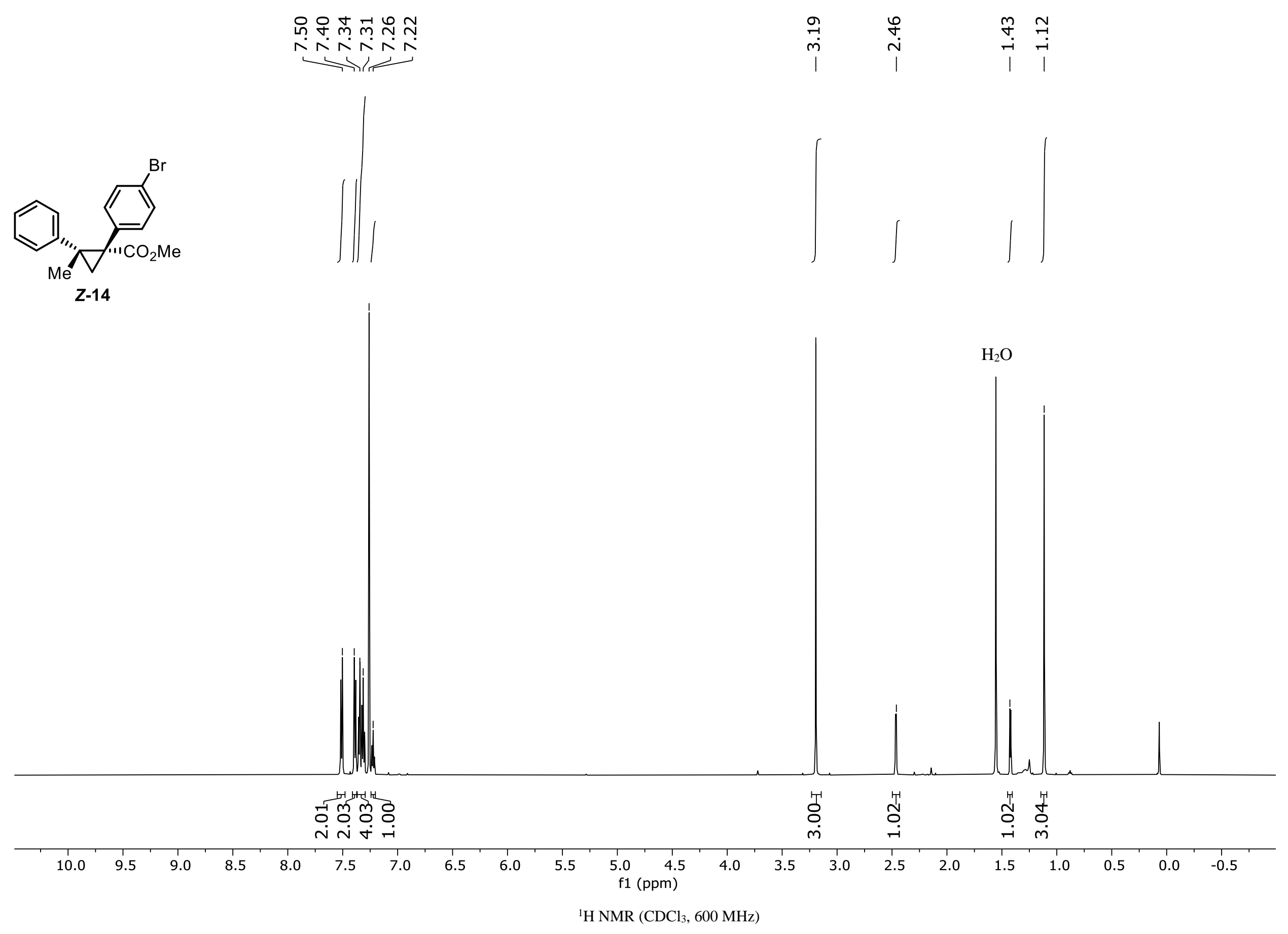



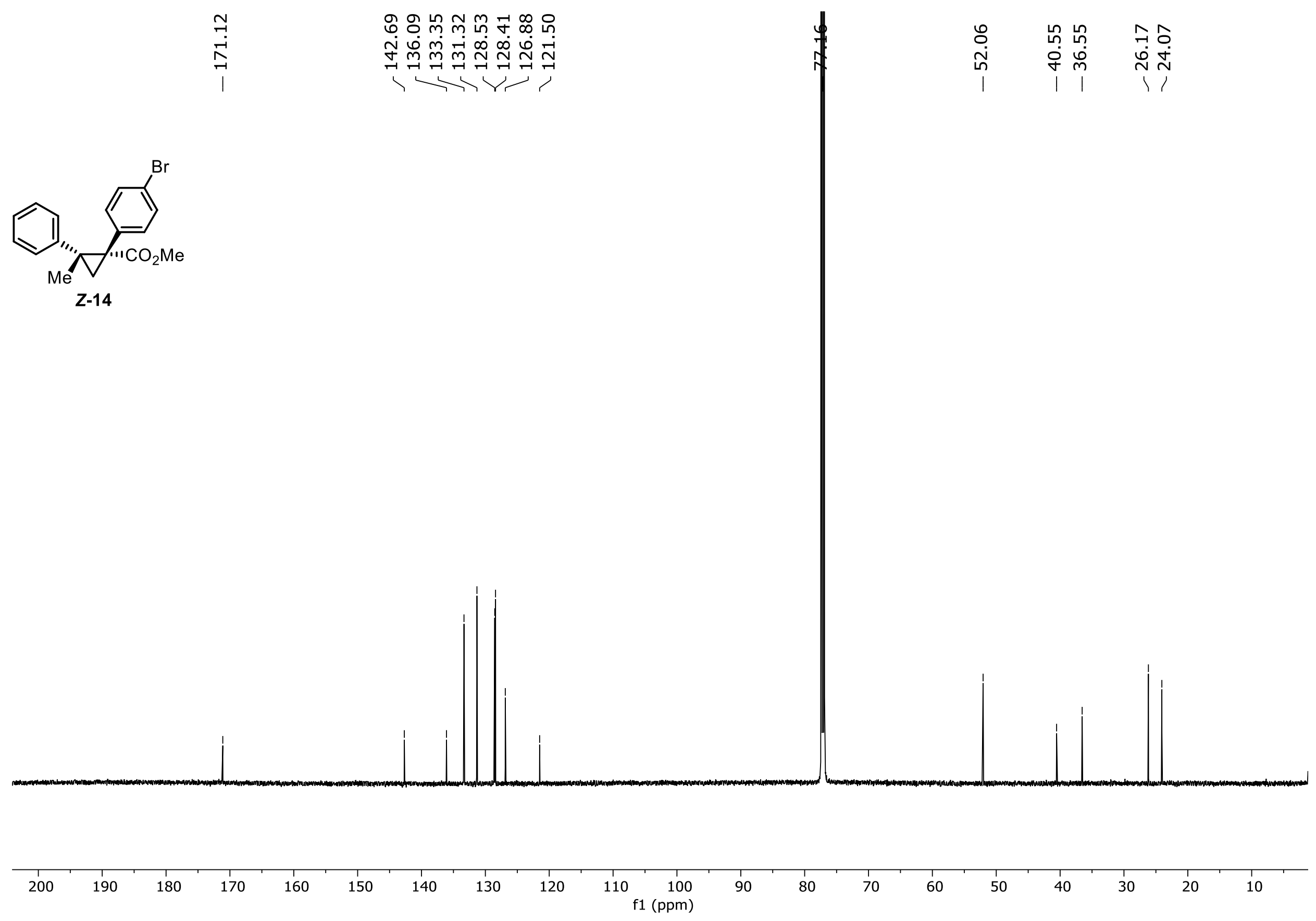

${ }^{1} \mathrm{H} \mathrm{NMR}\left(\mathrm{CDCl}_{3}, 151 \mathrm{MHz}\right)$ 


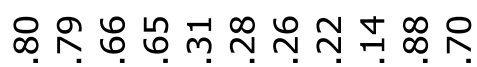

NNNNNNNベ

${ }_{1}$
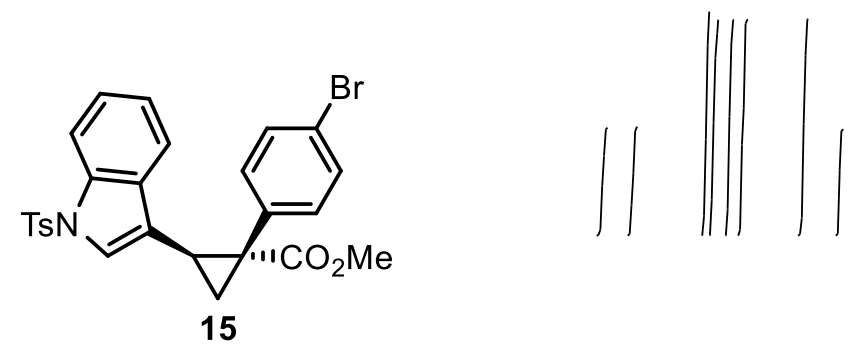

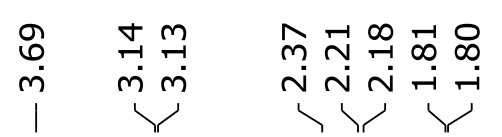
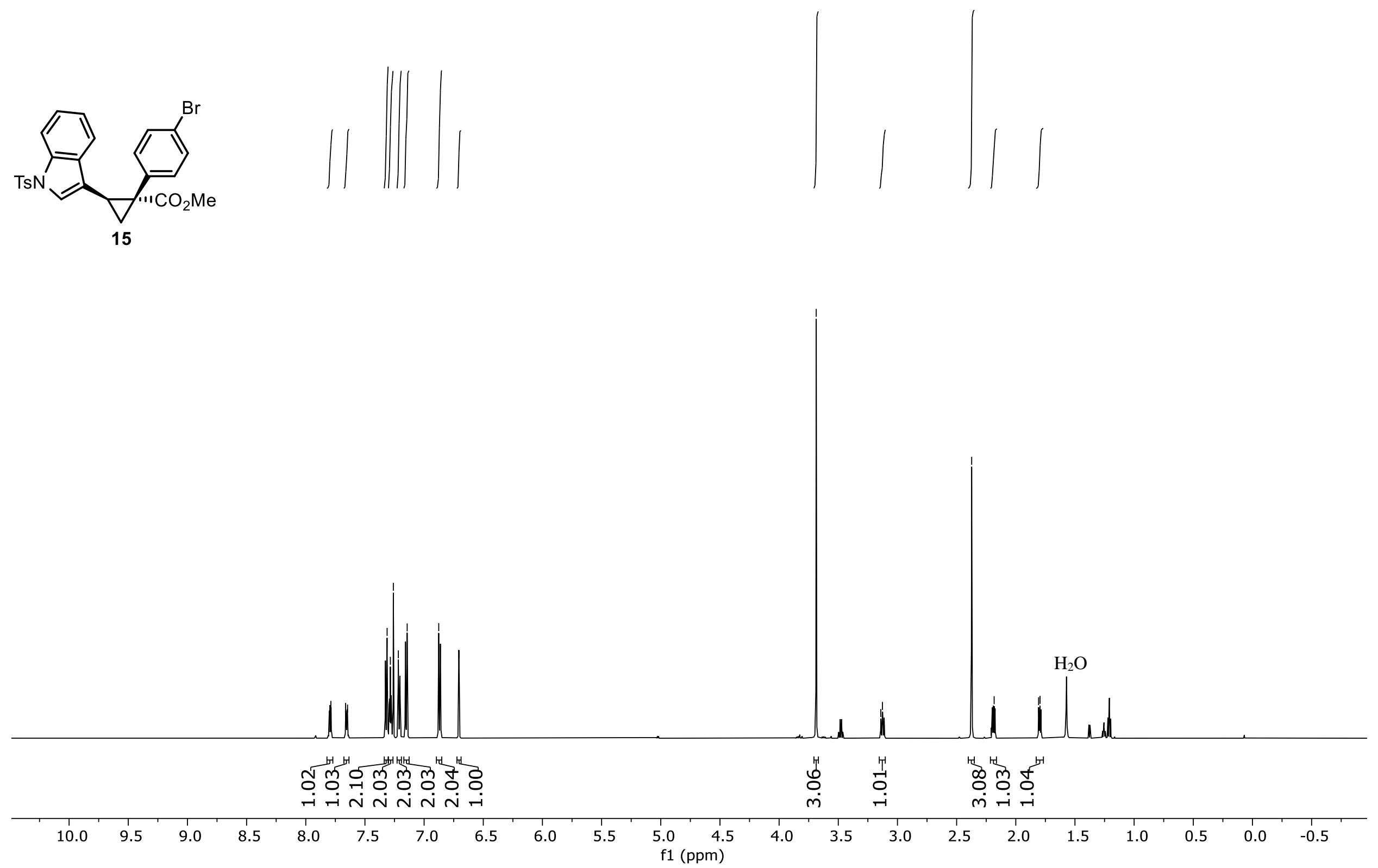

${ }^{1} \mathrm{H} \mathrm{NMR}\left(\mathrm{CDCl}_{3}, 600 \mathrm{MHz}\right)$ 

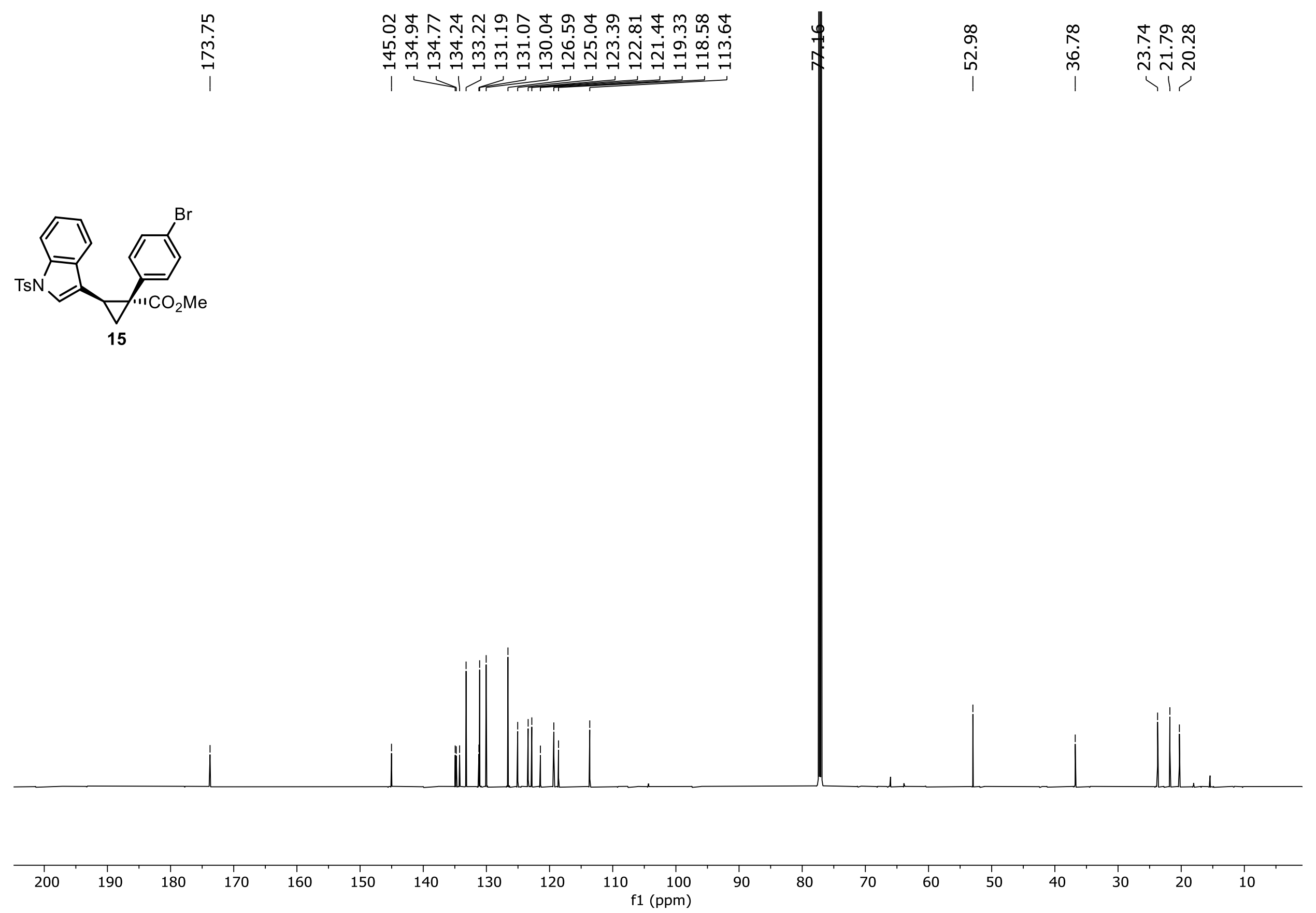

${ }^{1} \mathrm{H} \mathrm{NMR}\left(\mathrm{CDCl}_{3}, 151 \mathrm{MHz}\right)$ 


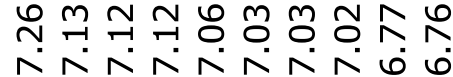

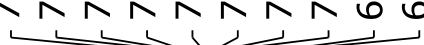


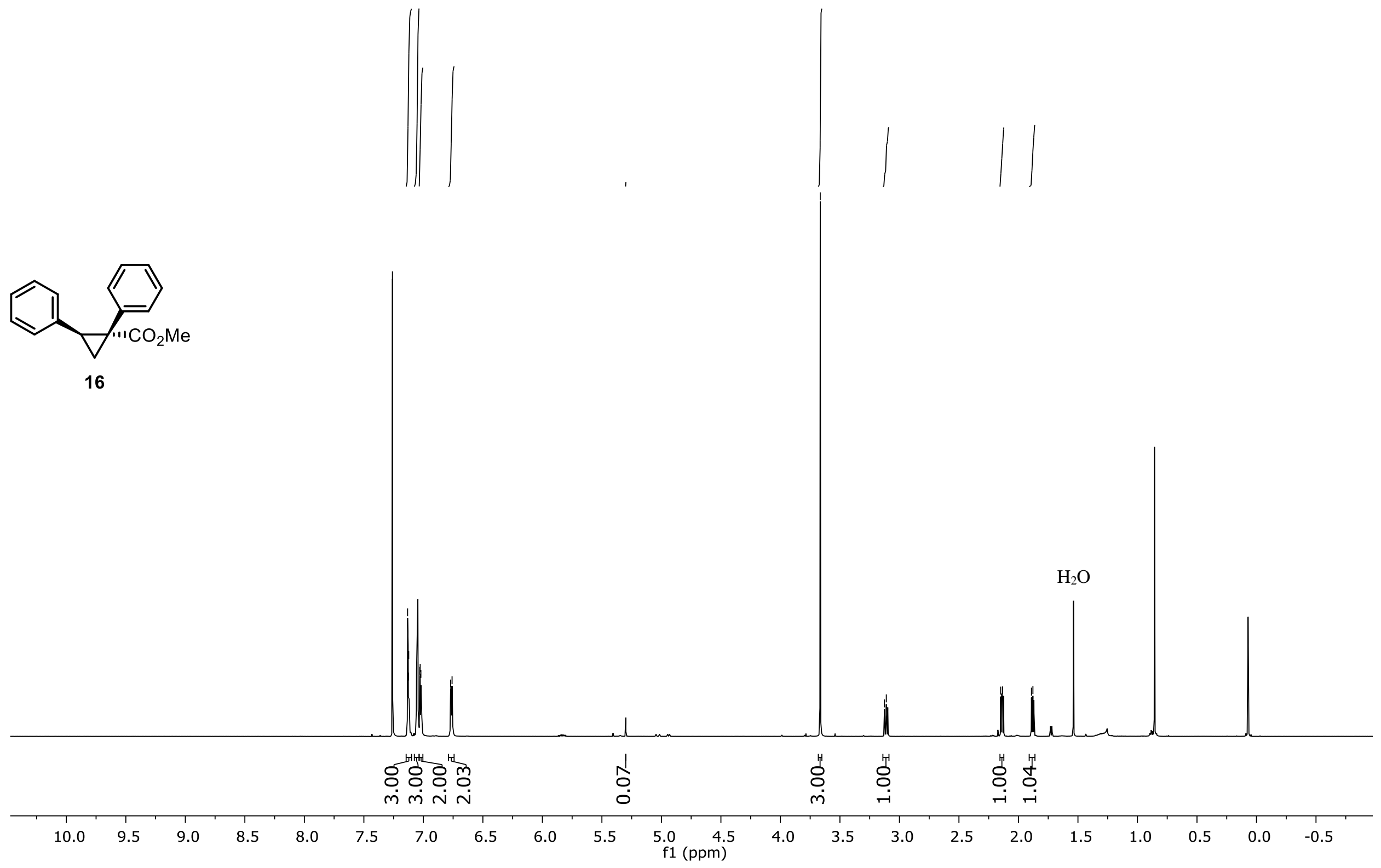

${ }^{1} \mathrm{H} \mathrm{NMR}\left(\mathrm{CDCl}_{3}, 600 \mathrm{MHz}\right)$ 


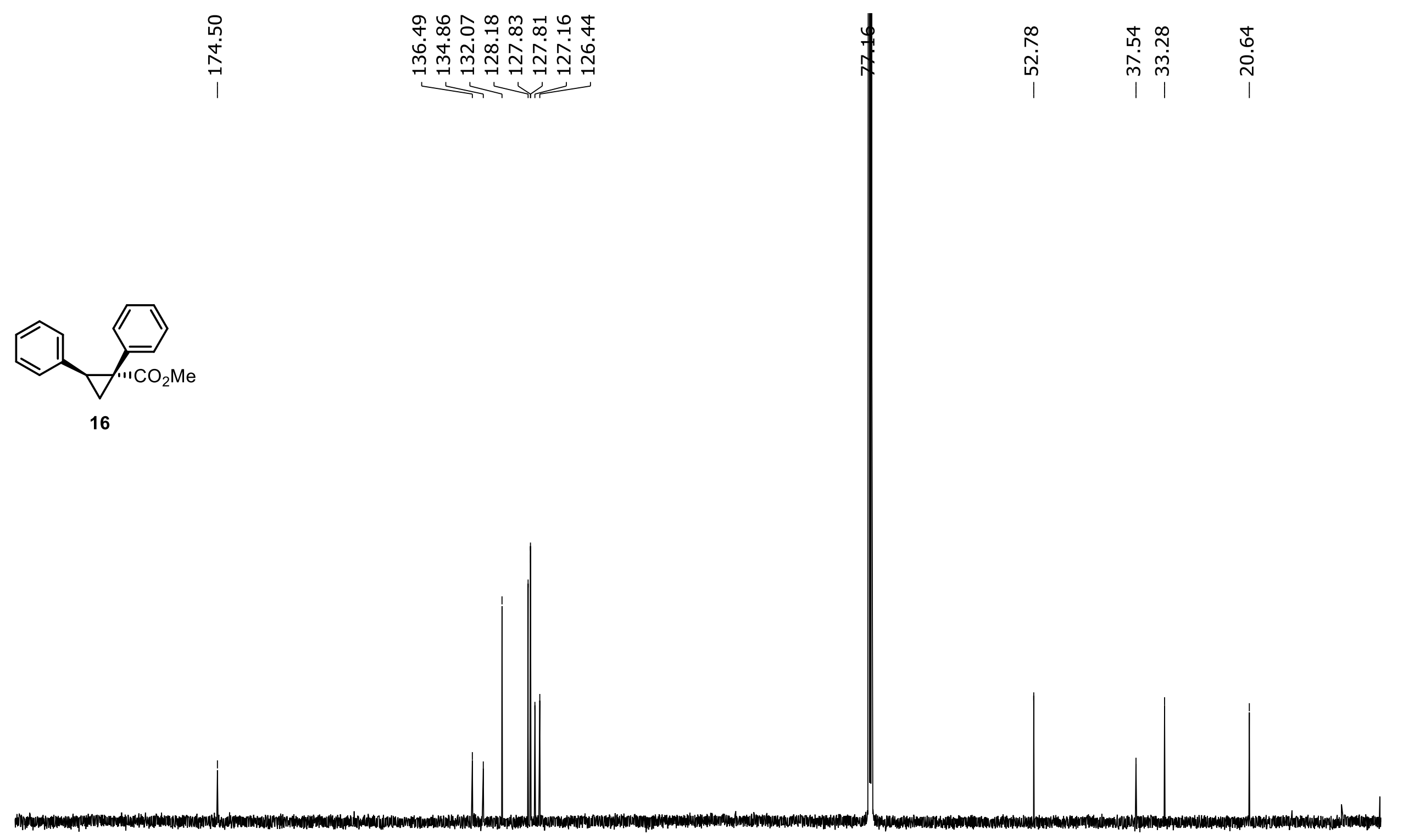

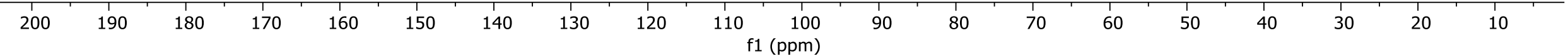

${ }^{13} \mathrm{C} \mathrm{NMR}\left(\mathrm{CDCl}_{3}, 151 \mathrm{MHz}\right)$ 
ข้ํำ

ก

$\checkmark>$

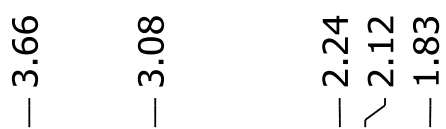
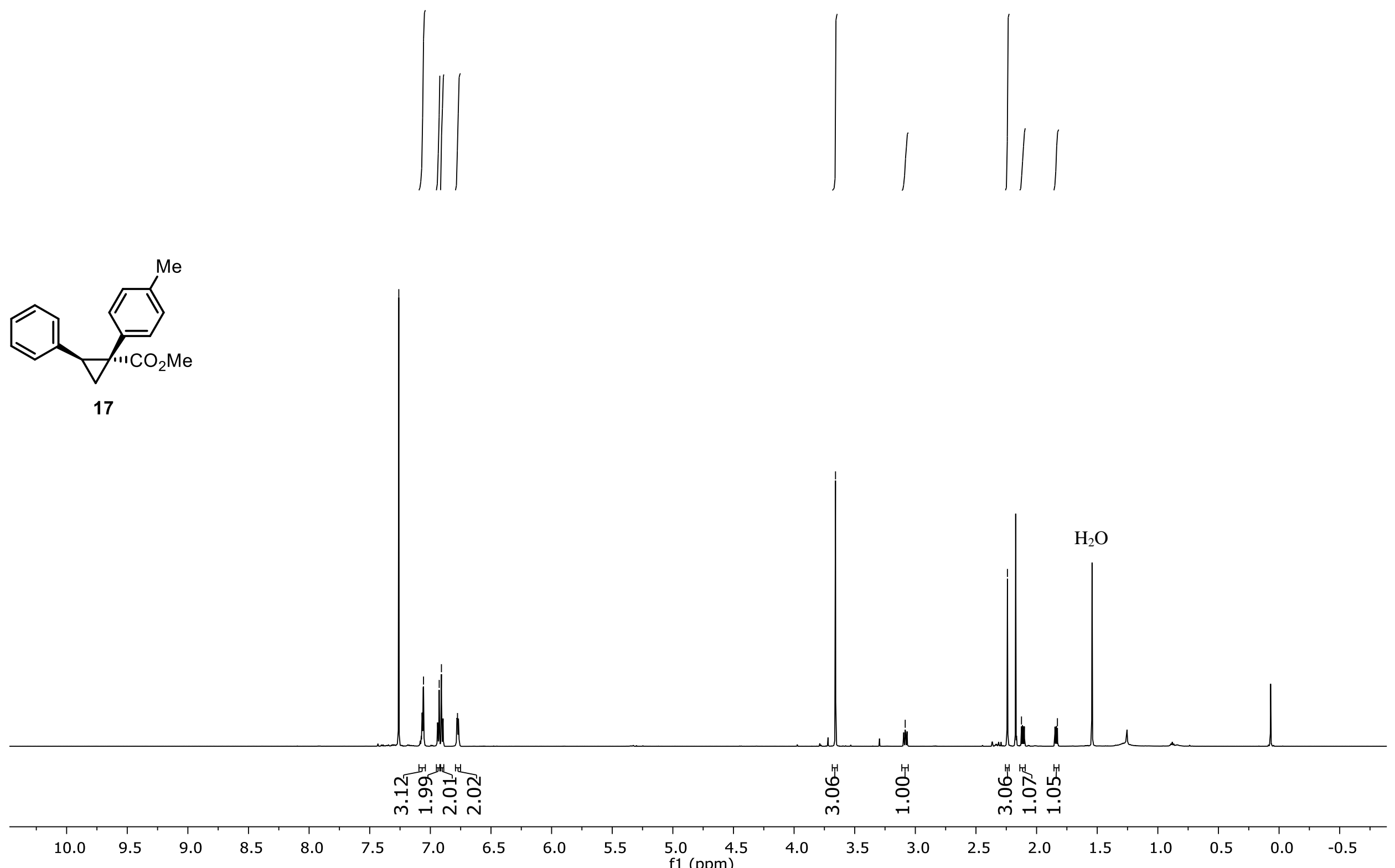

${ }^{1} \mathrm{H} \mathrm{NMR}\left(\mathrm{CDCl}_{3}, 400 \mathrm{MHz}\right)$ 


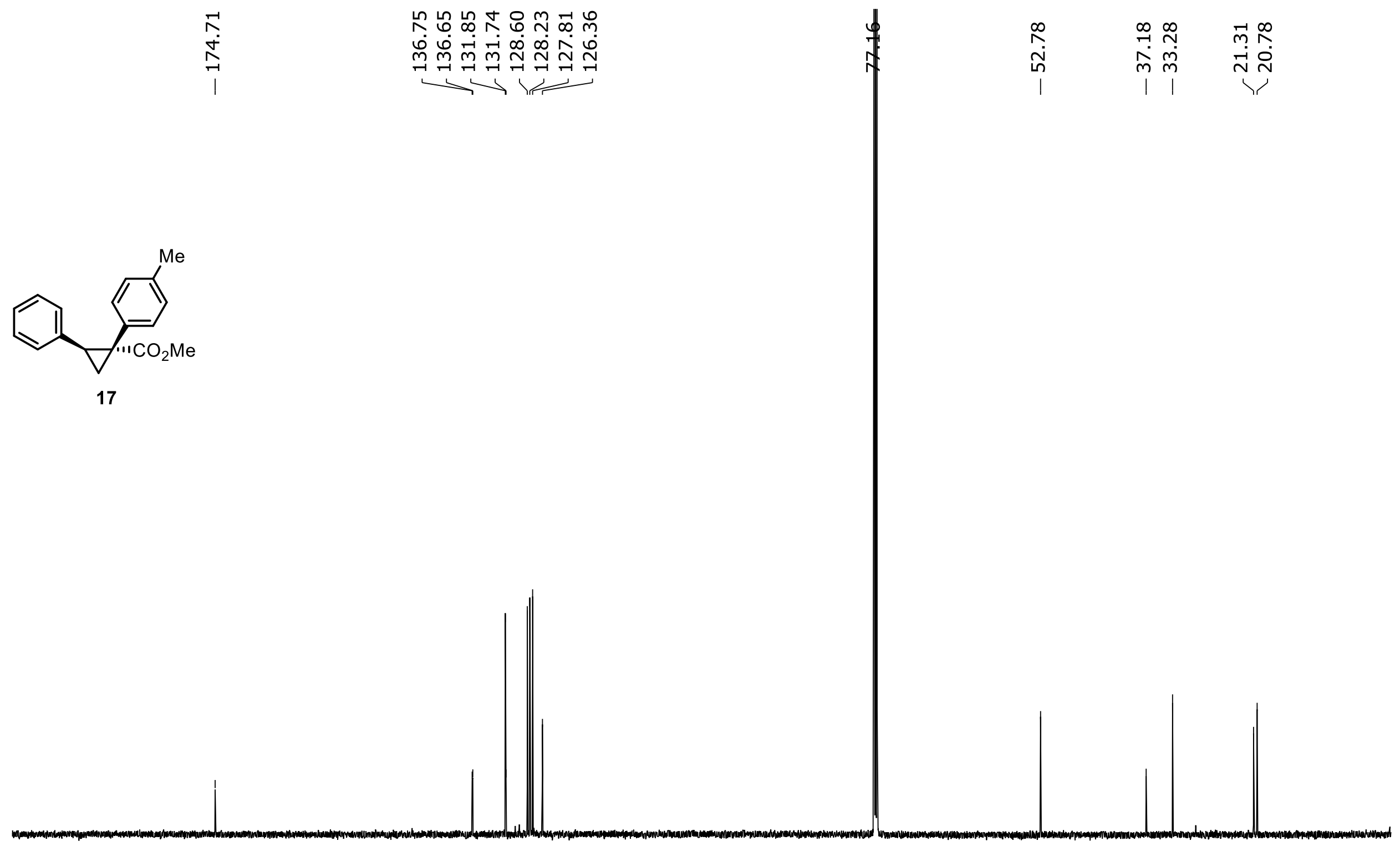

${ }^{13} \mathrm{C} \mathrm{NMR}\left(\mathrm{CDCl}_{3}, 151 \mathrm{MHz}\right)$ 


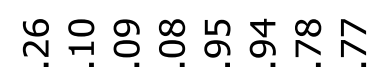

הNヘN

(1)
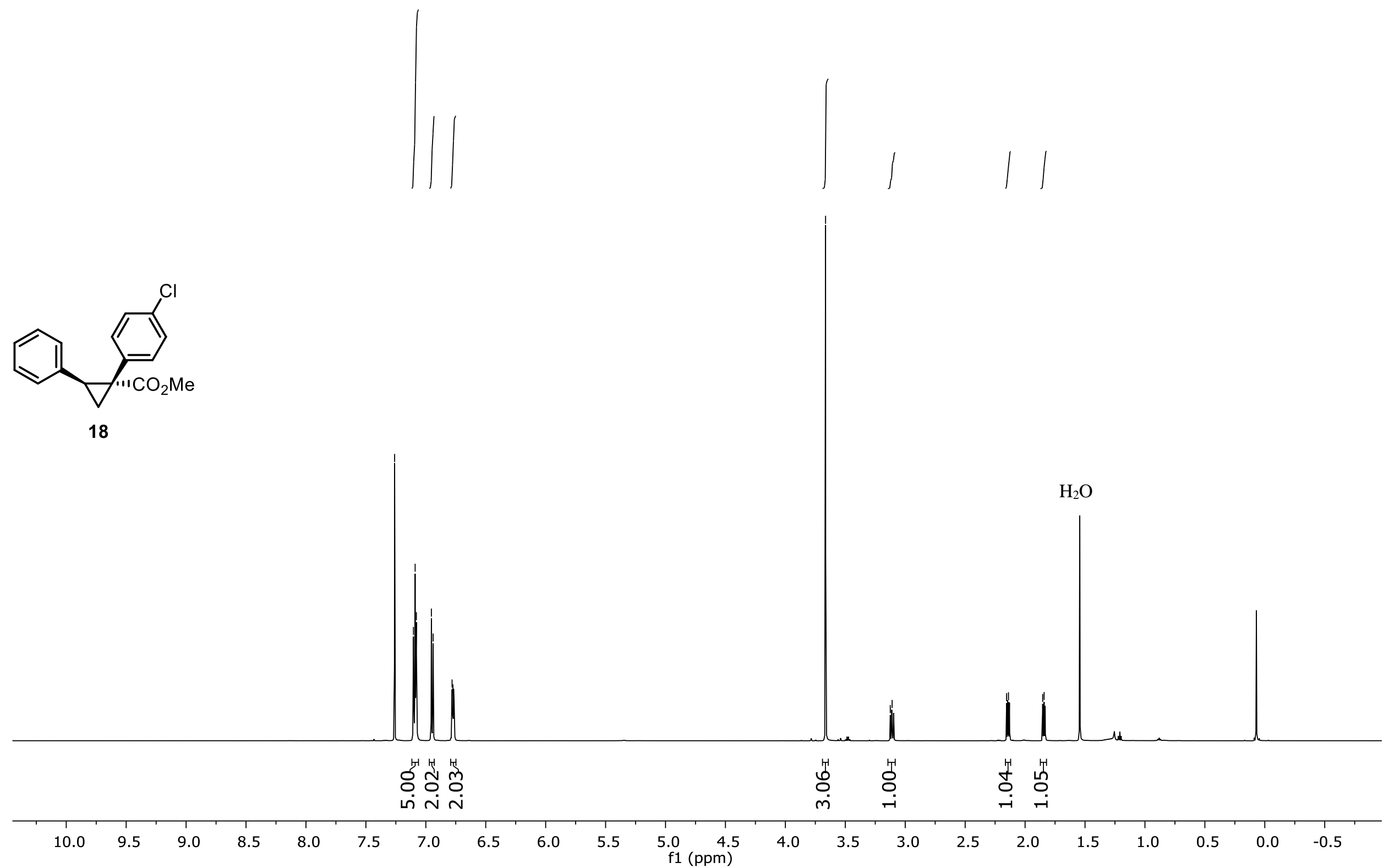

${ }^{1} \mathrm{H} \mathrm{NMR}\left(\mathrm{CDCl}_{3}, 600 \mathrm{MHz}\right)$ m

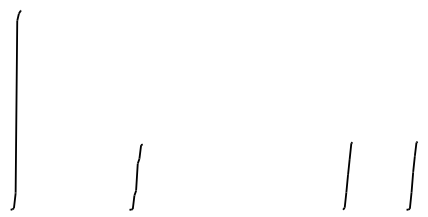




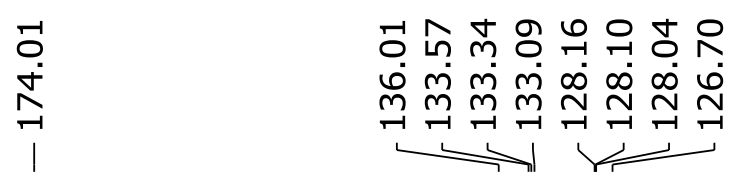

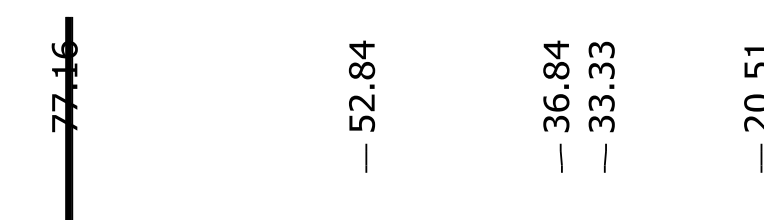

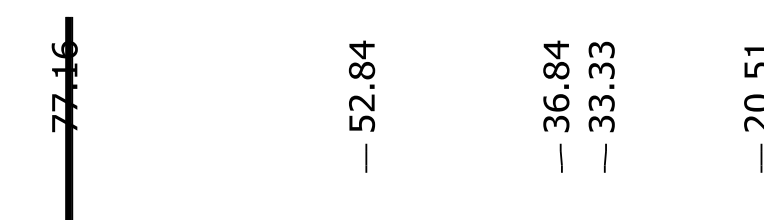

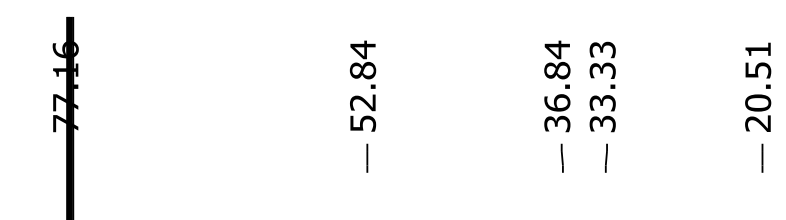
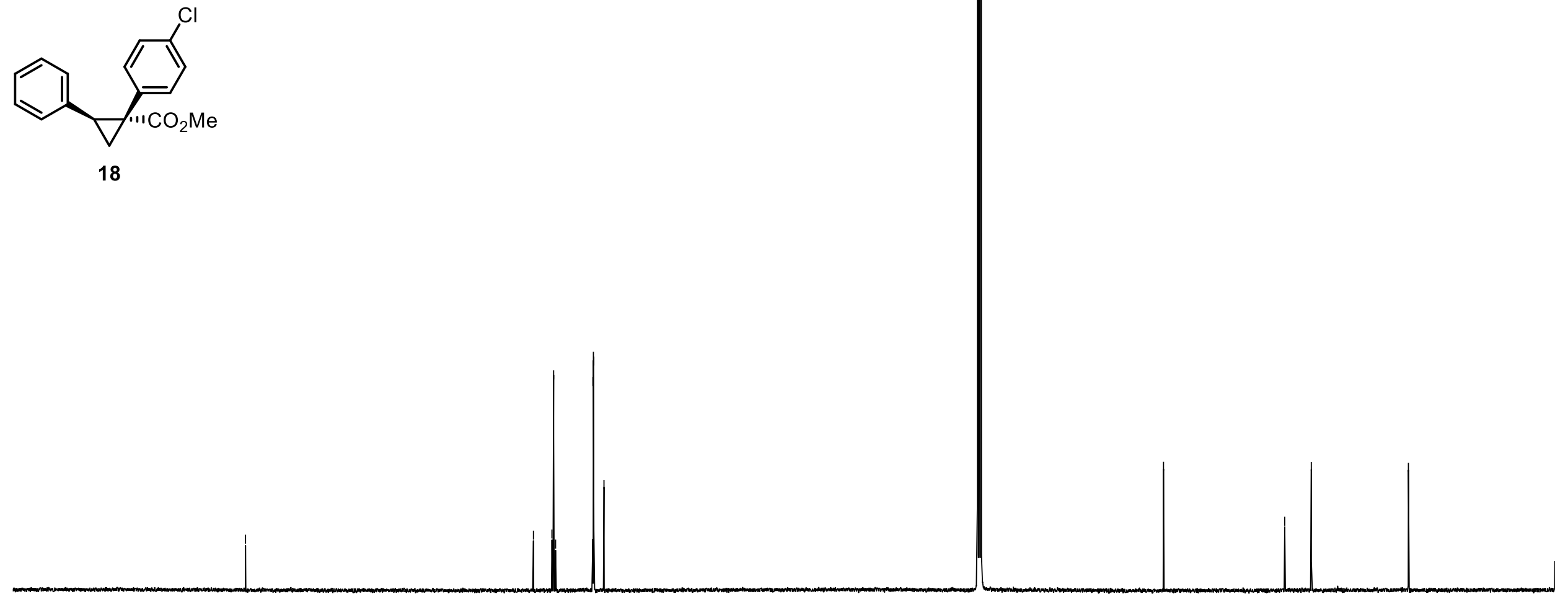

200

190

${ }^{13} \mathrm{C} \mathrm{NMR}\left(\mathrm{CDCl}_{3}, 151 \mathrm{MHz}\right)$ 


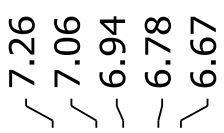

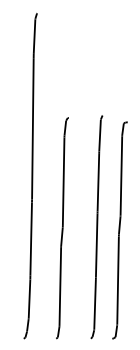

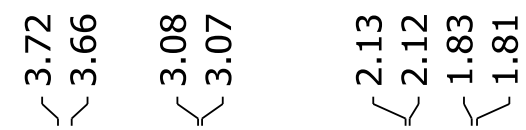

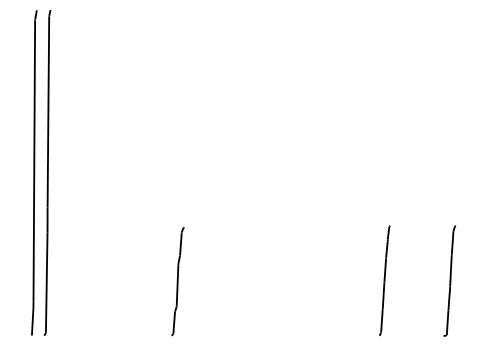

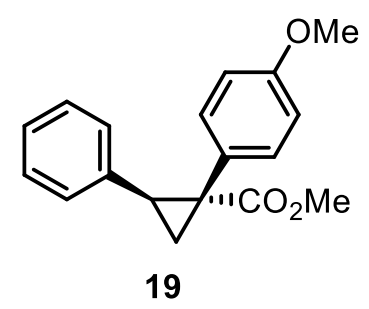

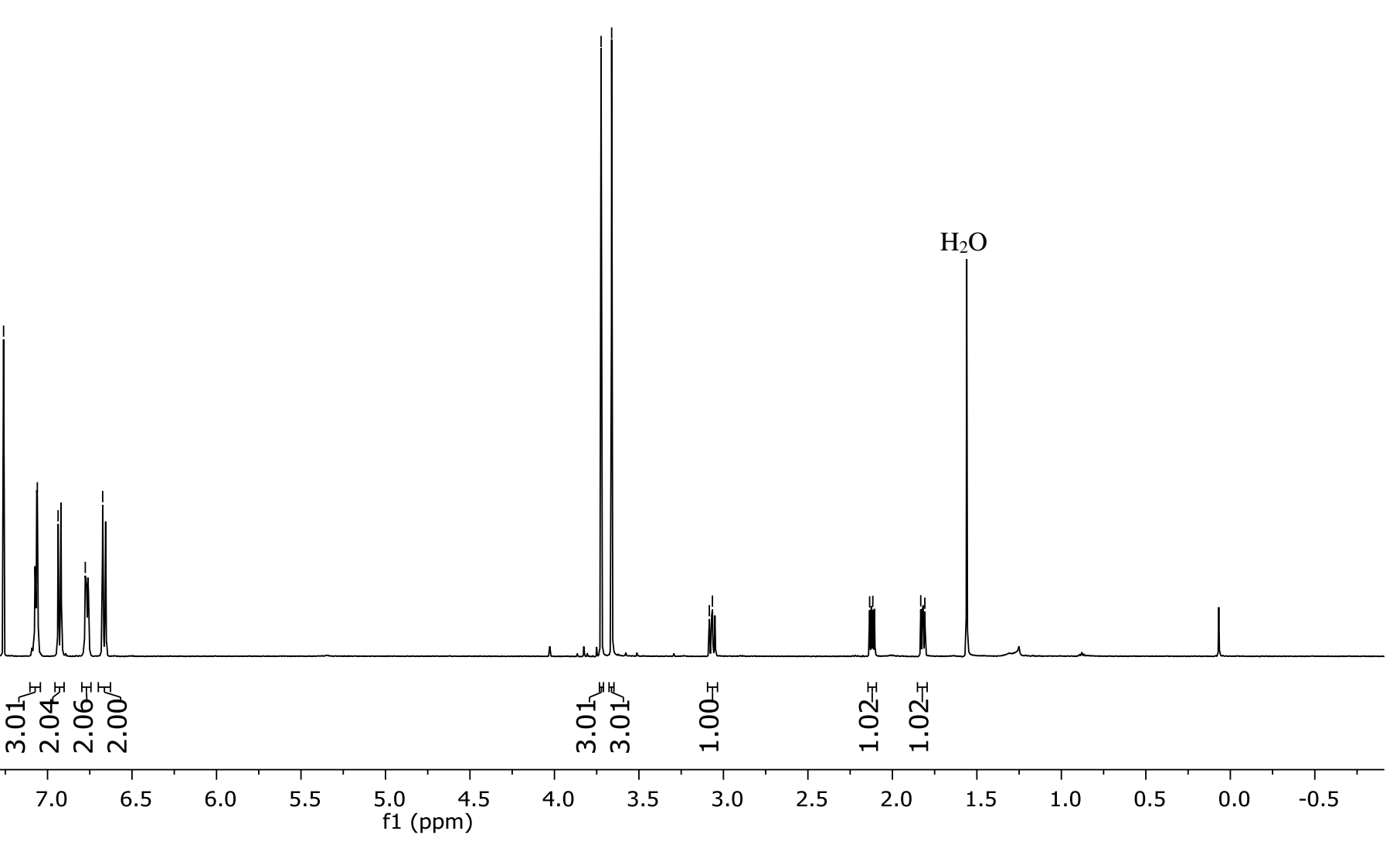

${ }^{1} \mathrm{H} \mathrm{NMR}\left(\mathrm{CDCl}_{3}, 600 \mathrm{MHz}\right)$ 

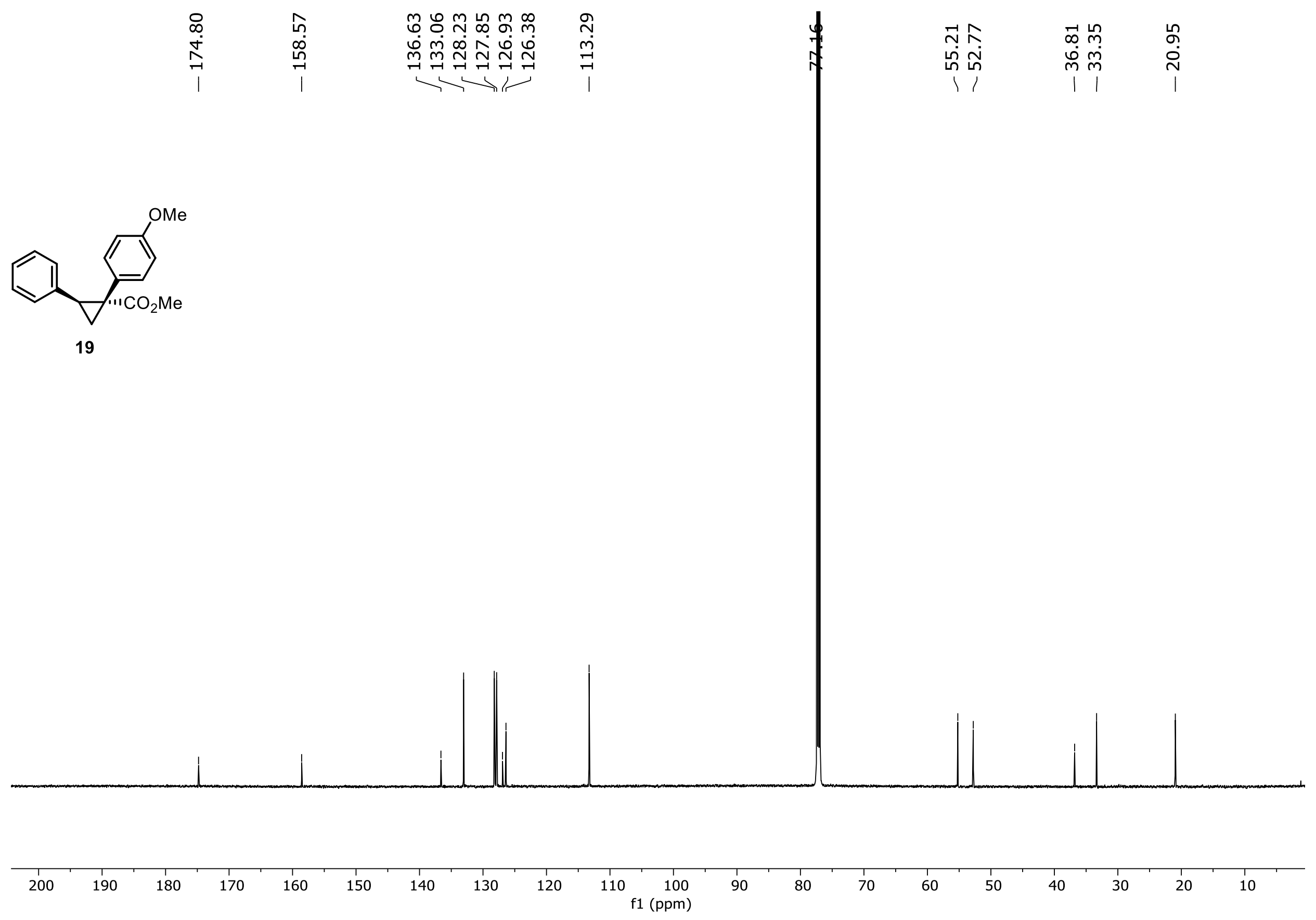

${ }^{1} \mathrm{H} \mathrm{NMR}\left(\mathrm{CDCl}_{3}, 151 \mathrm{MHz}\right)$ 


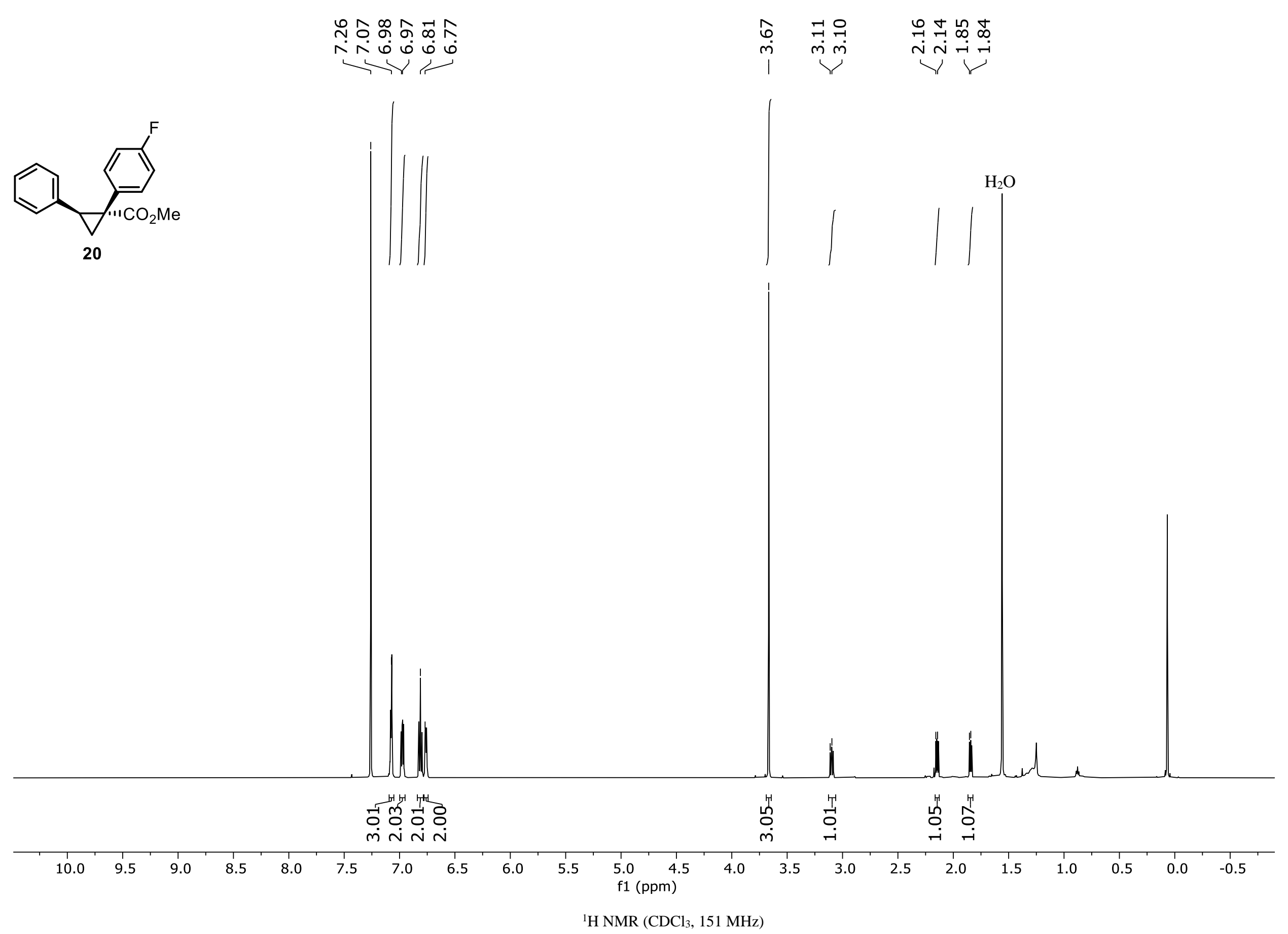



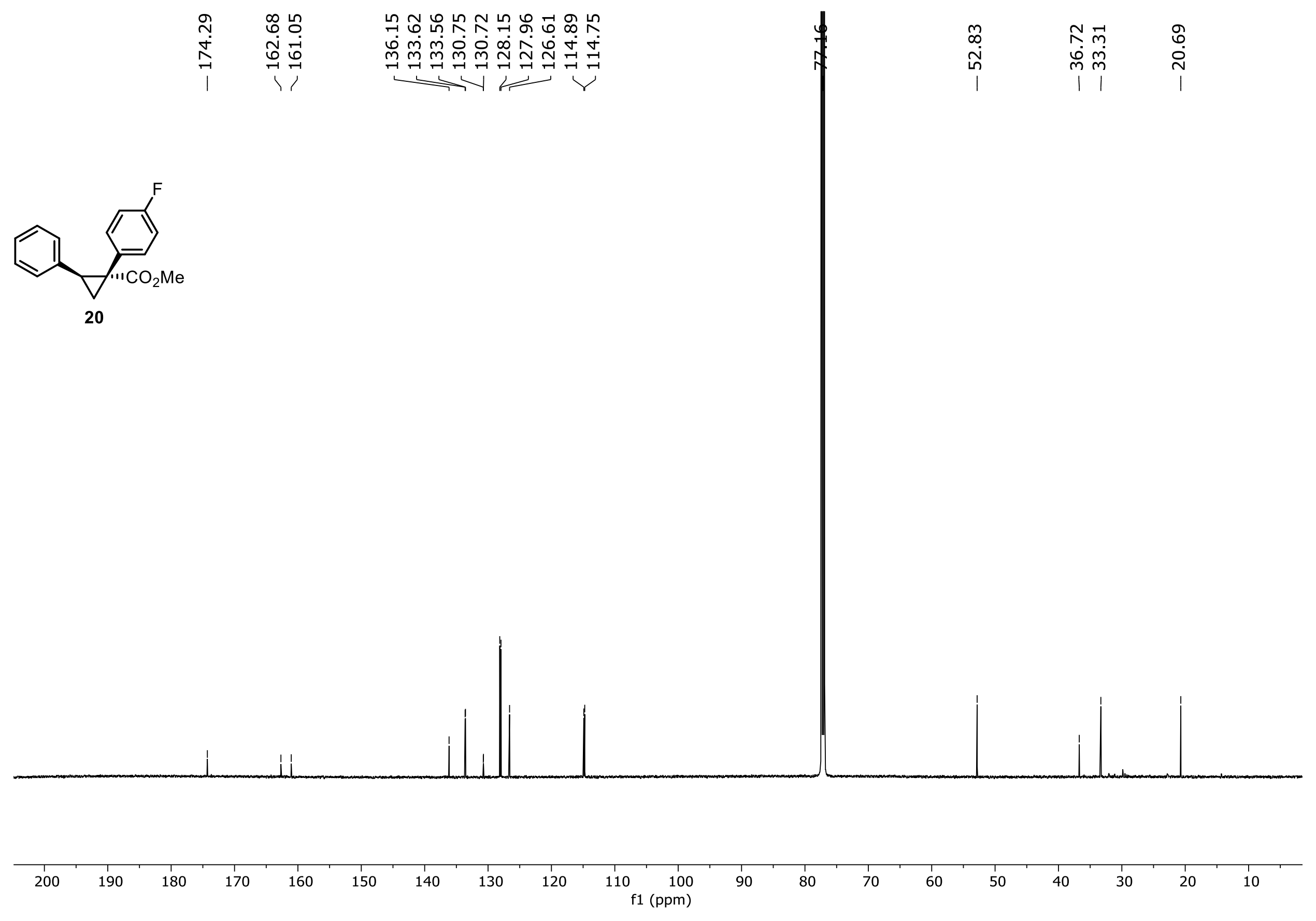

${ }^{1} \mathrm{H} \mathrm{NMR}\left(\mathrm{CDCl}_{3}, 151 \mathrm{MHz}\right)$ 


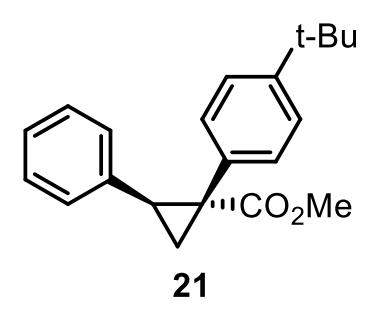

\।

ले

त
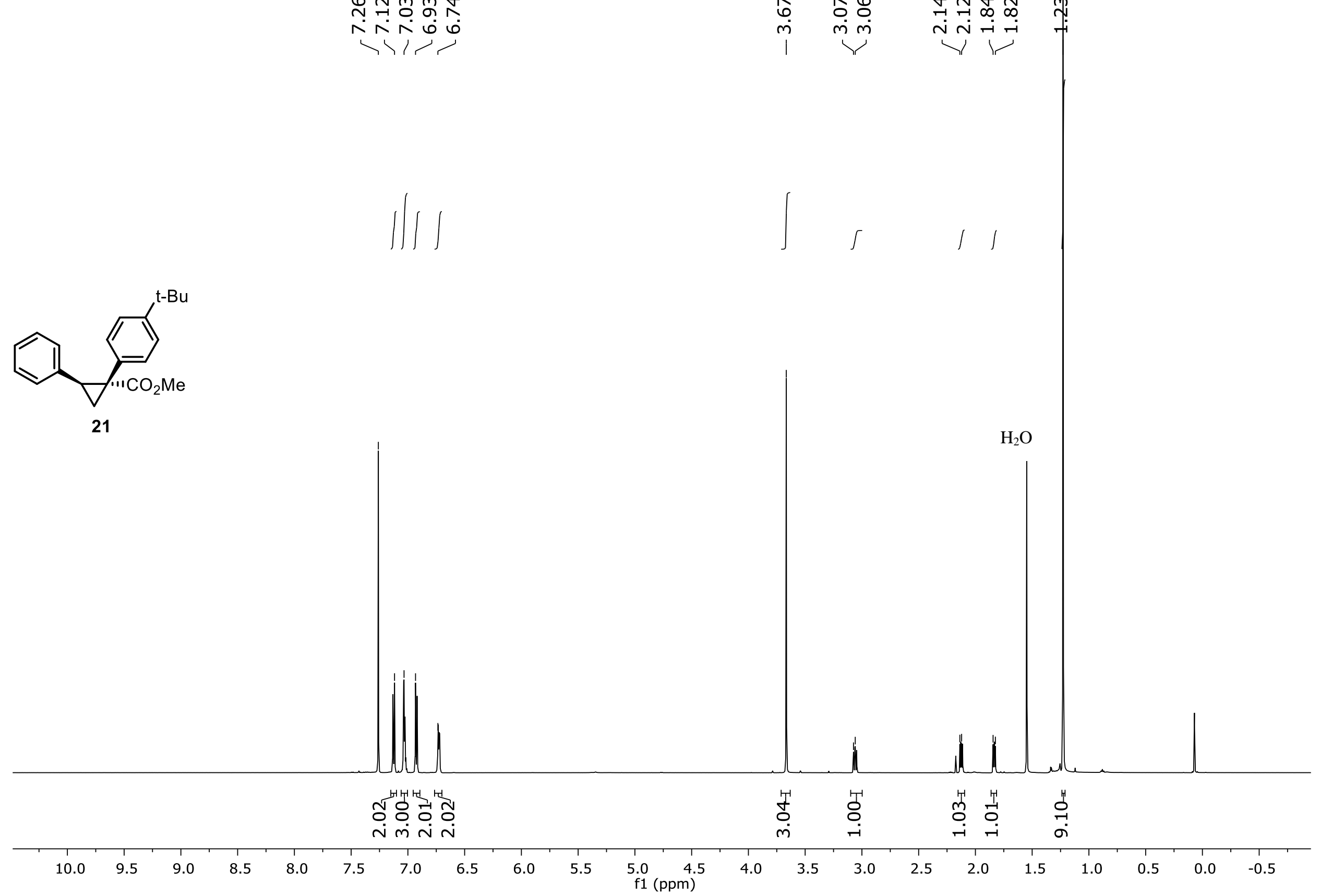

${ }^{1} \mathrm{H} \mathrm{NMR}\left(\mathrm{CDCl}_{3}, 600 \mathrm{MHz}\right)$ 


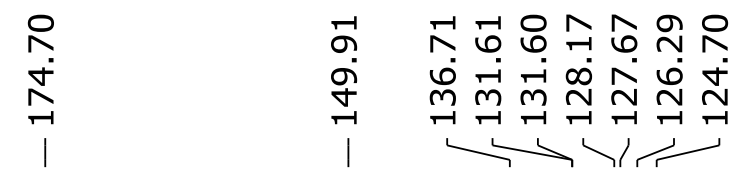

永

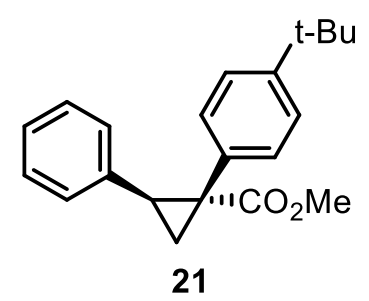

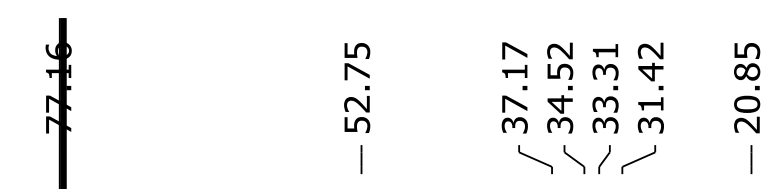

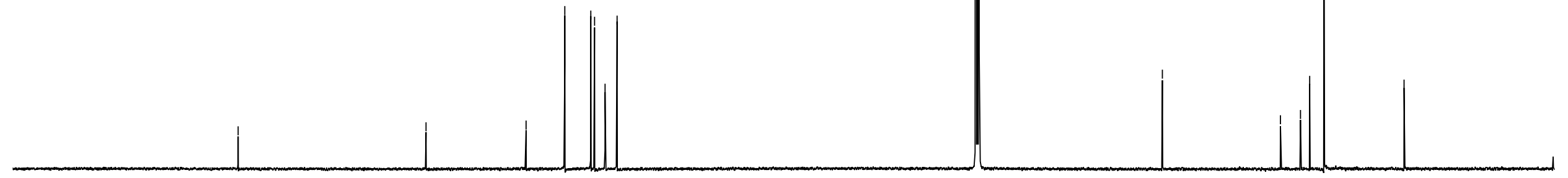

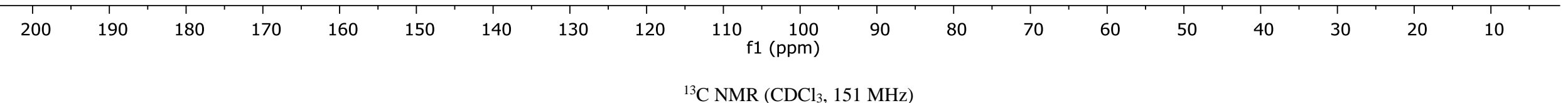




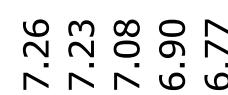

הN

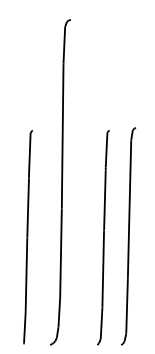

m
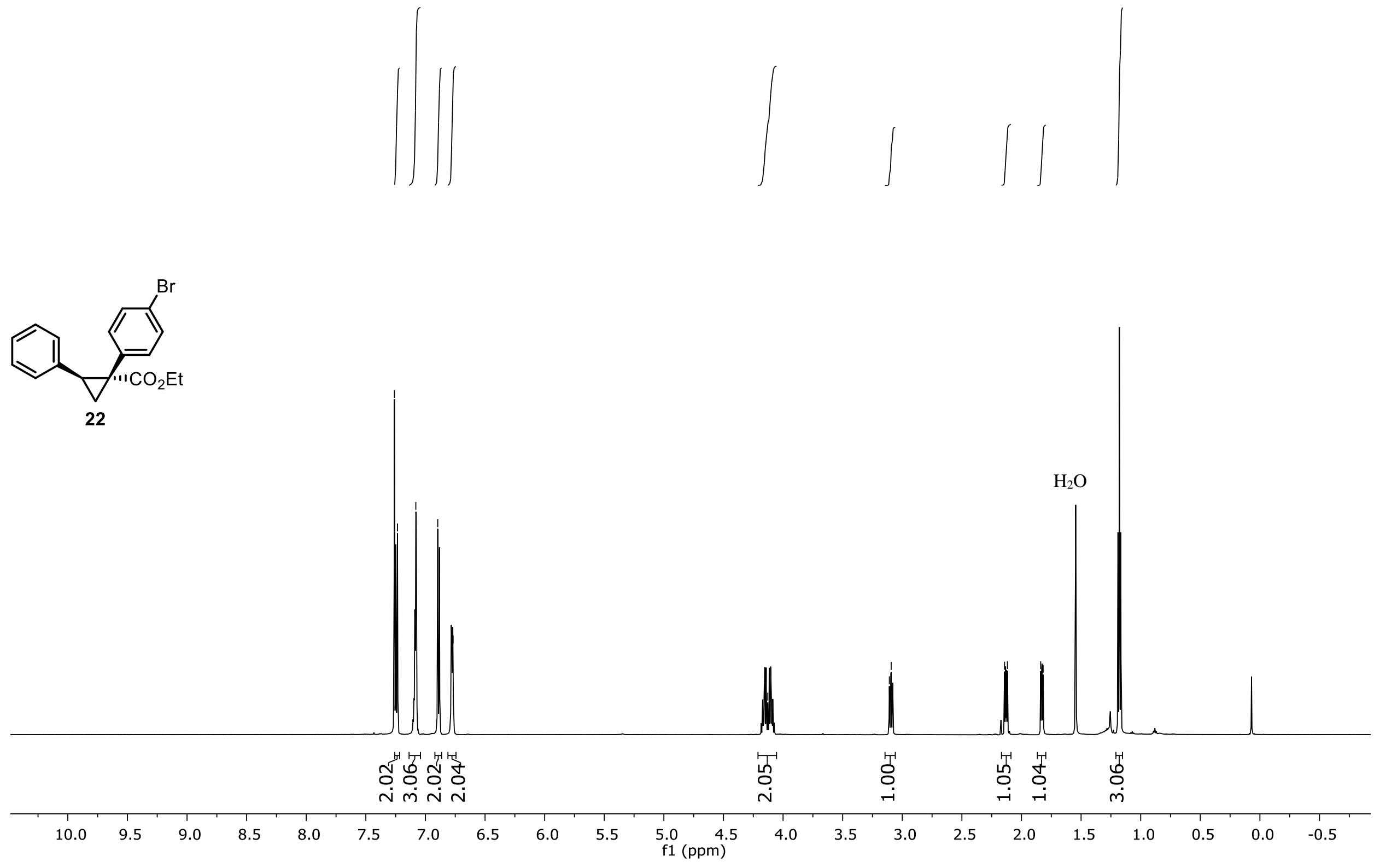

${ }^{1} \mathrm{H} \mathrm{NMR}\left(\mathrm{CDCl}_{3}, 600 \mathrm{MHz}\right)$ 

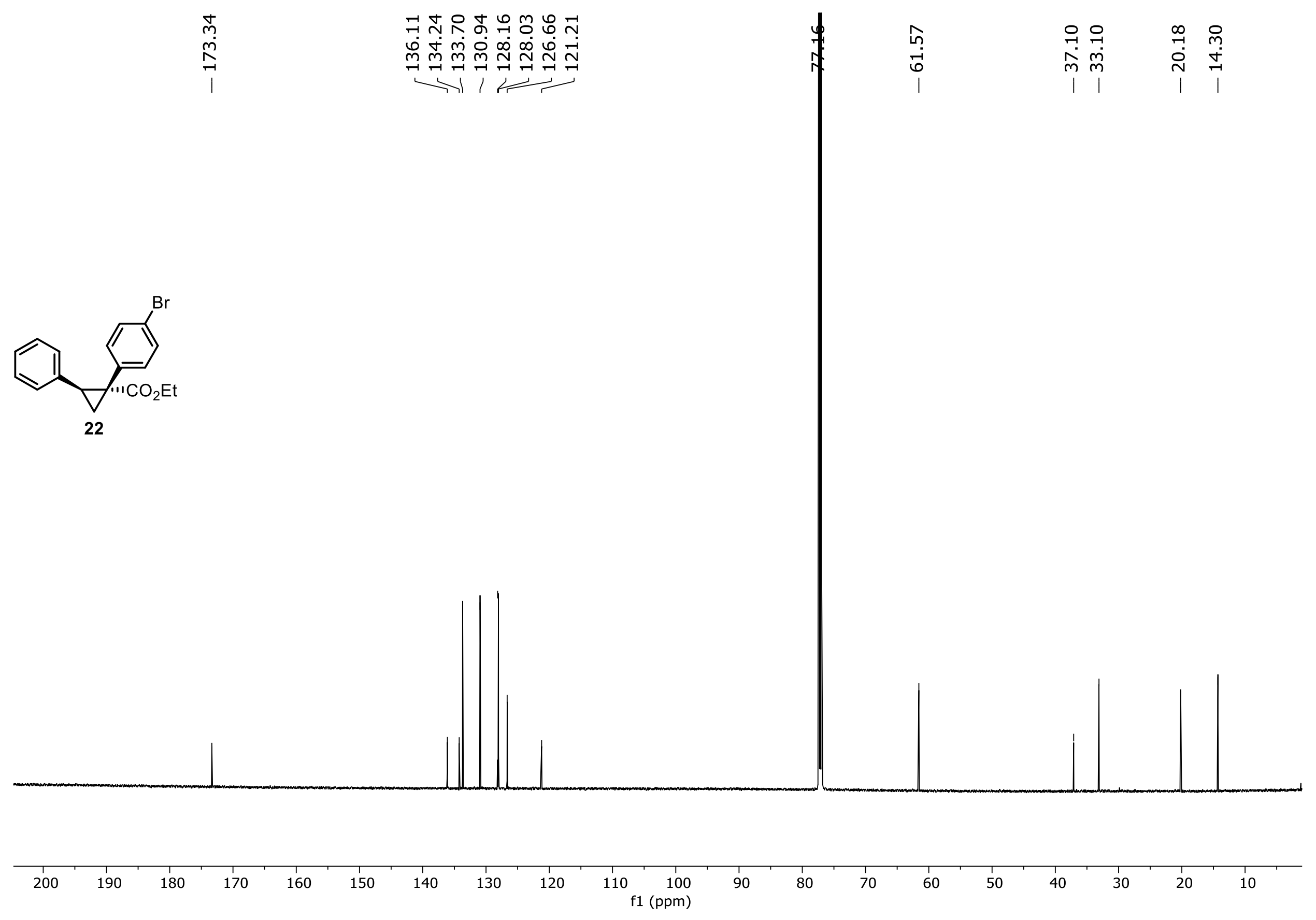

${ }^{13} \mathrm{C} \mathrm{NMR}\left(\mathrm{CDCl}_{3}, 151 \mathrm{MHz}\right)$ 


\begin{tabular}{|c|c|c|c|}
\hline 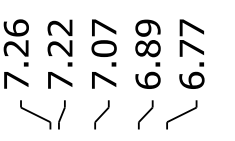 & 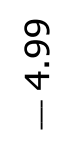 & 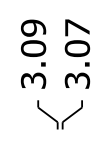 & تَ \\
\hline
\end{tabular}
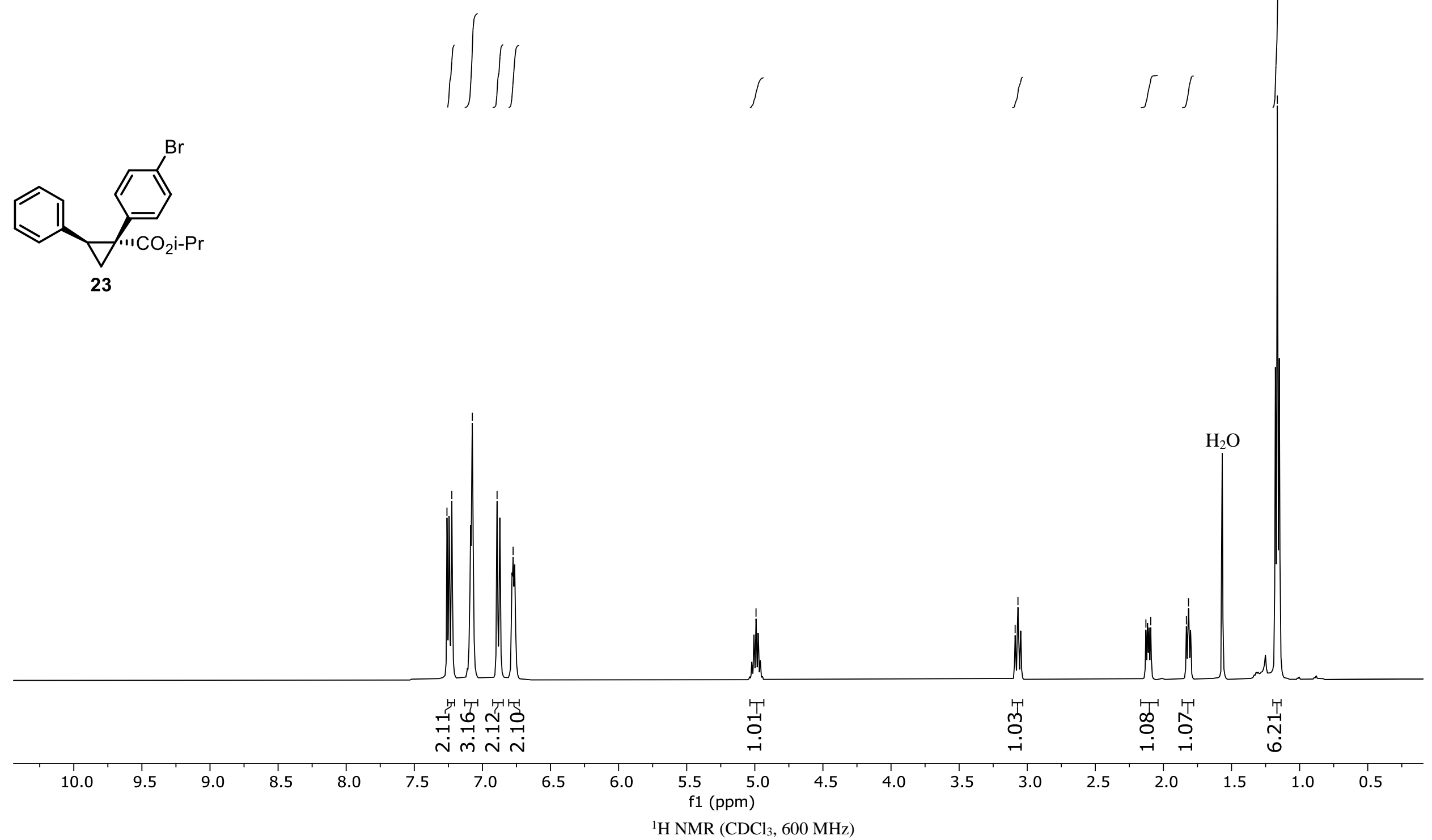


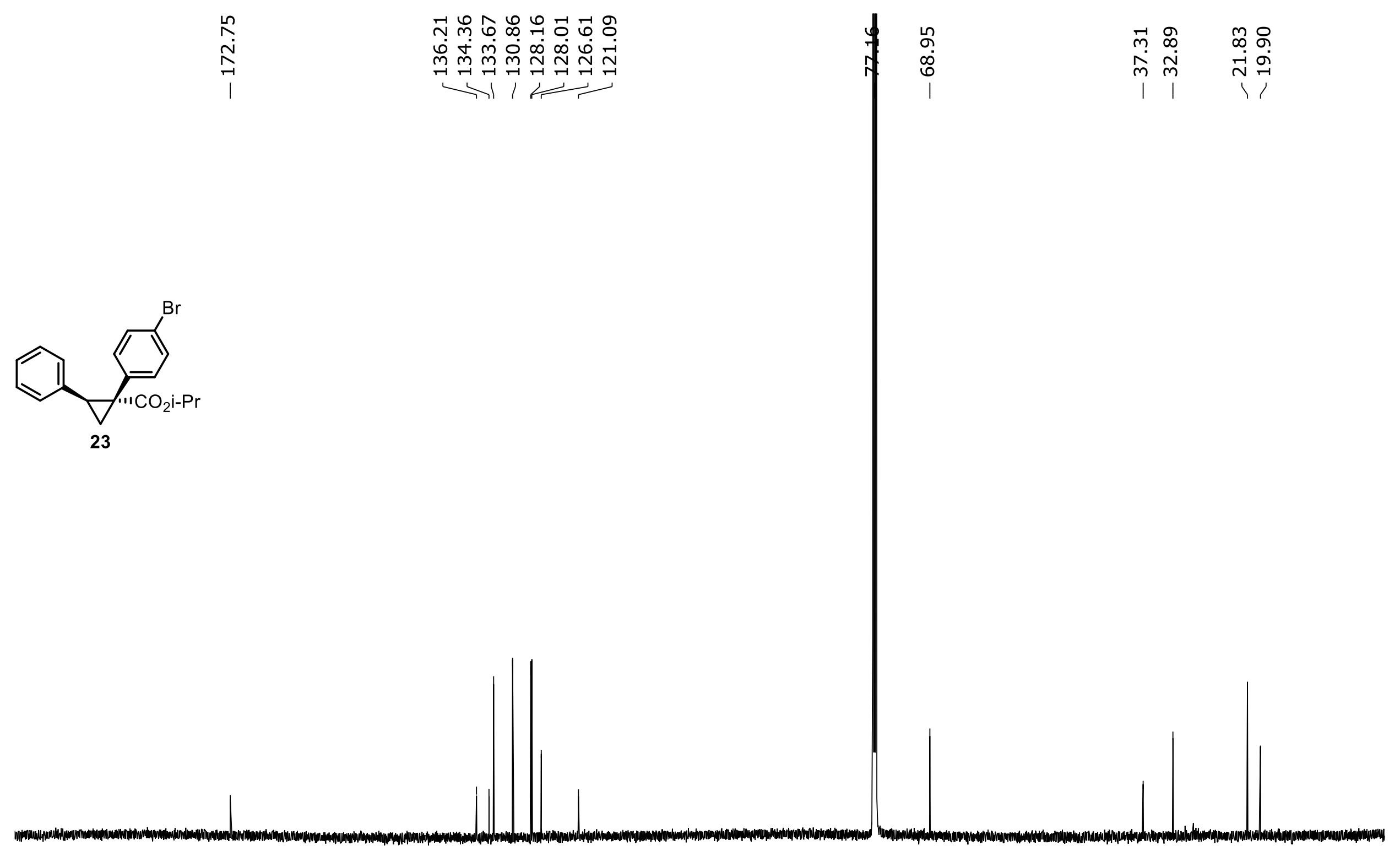

${ }^{13} \mathrm{C} \mathrm{NMR}\left(\mathrm{CDCl}_{3}, 151 \mathrm{MHz}\right)$ 


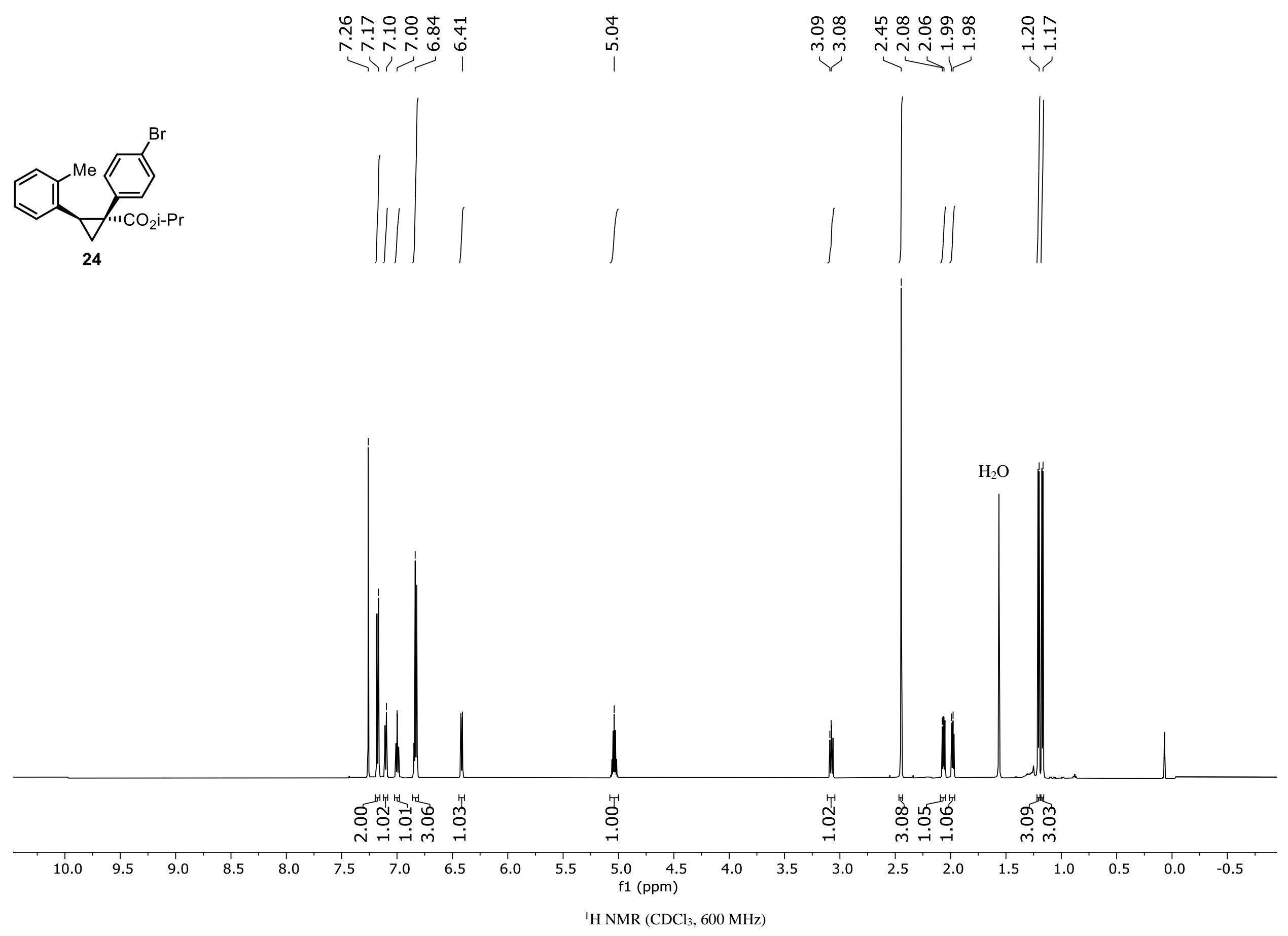



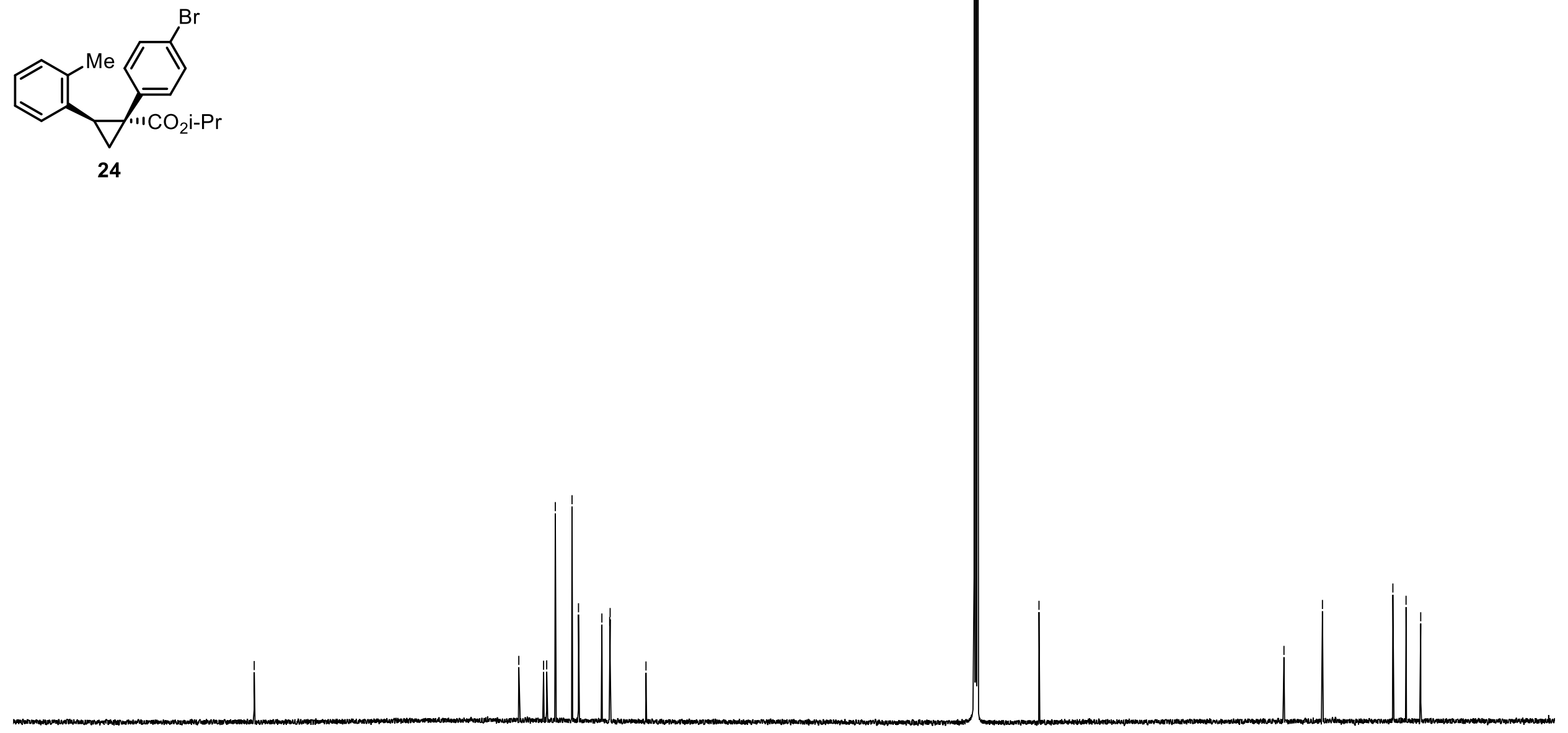

${ }^{1} \mathrm{H} \mathrm{NMR}\left(\mathrm{CDCl}_{3}, 151 \mathrm{MHz}\right)$ 


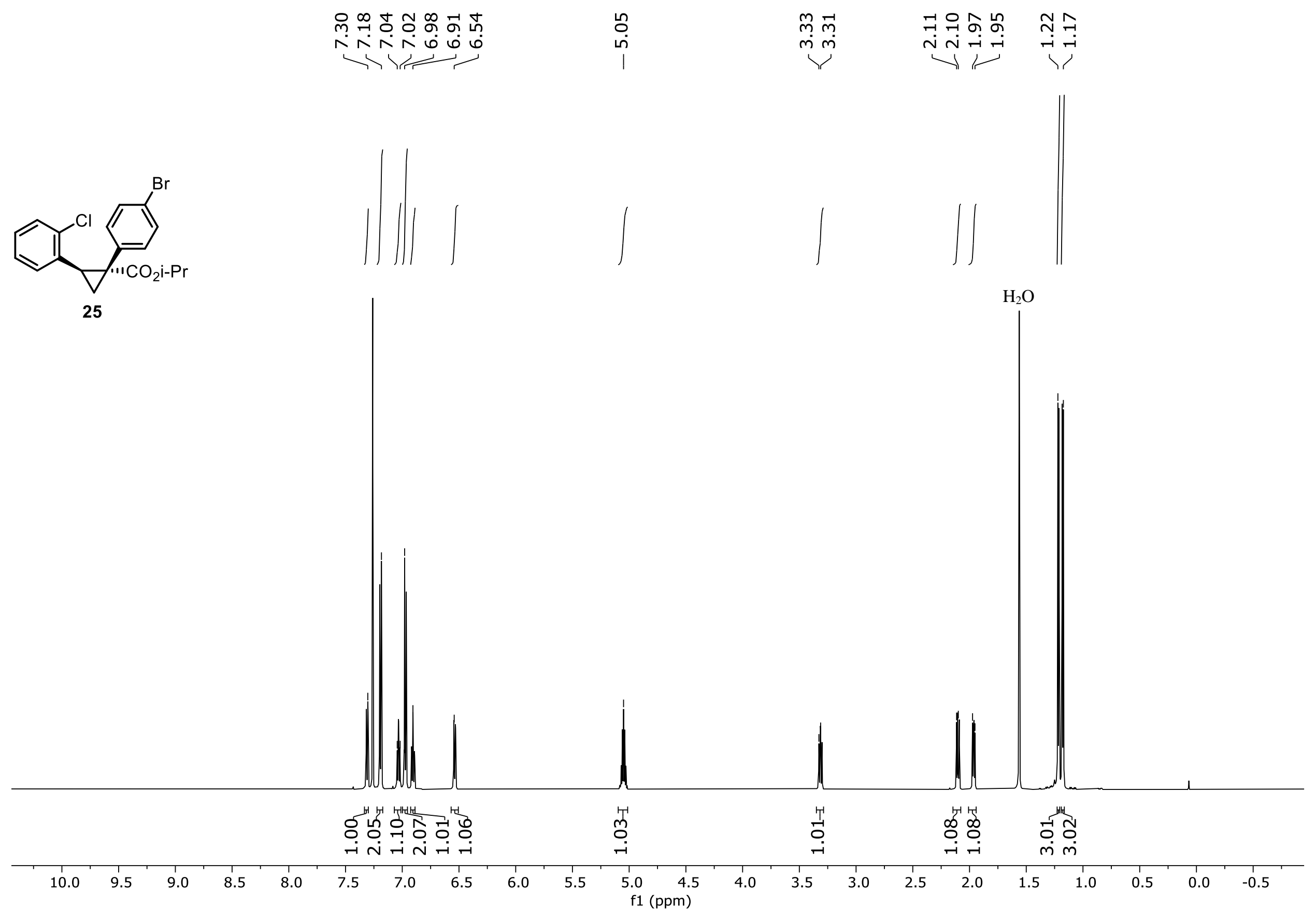

${ }^{1} \mathrm{H} \mathrm{NMR}\left(\mathrm{CDCl}_{3}, 600 \mathrm{MHz}\right)$ 


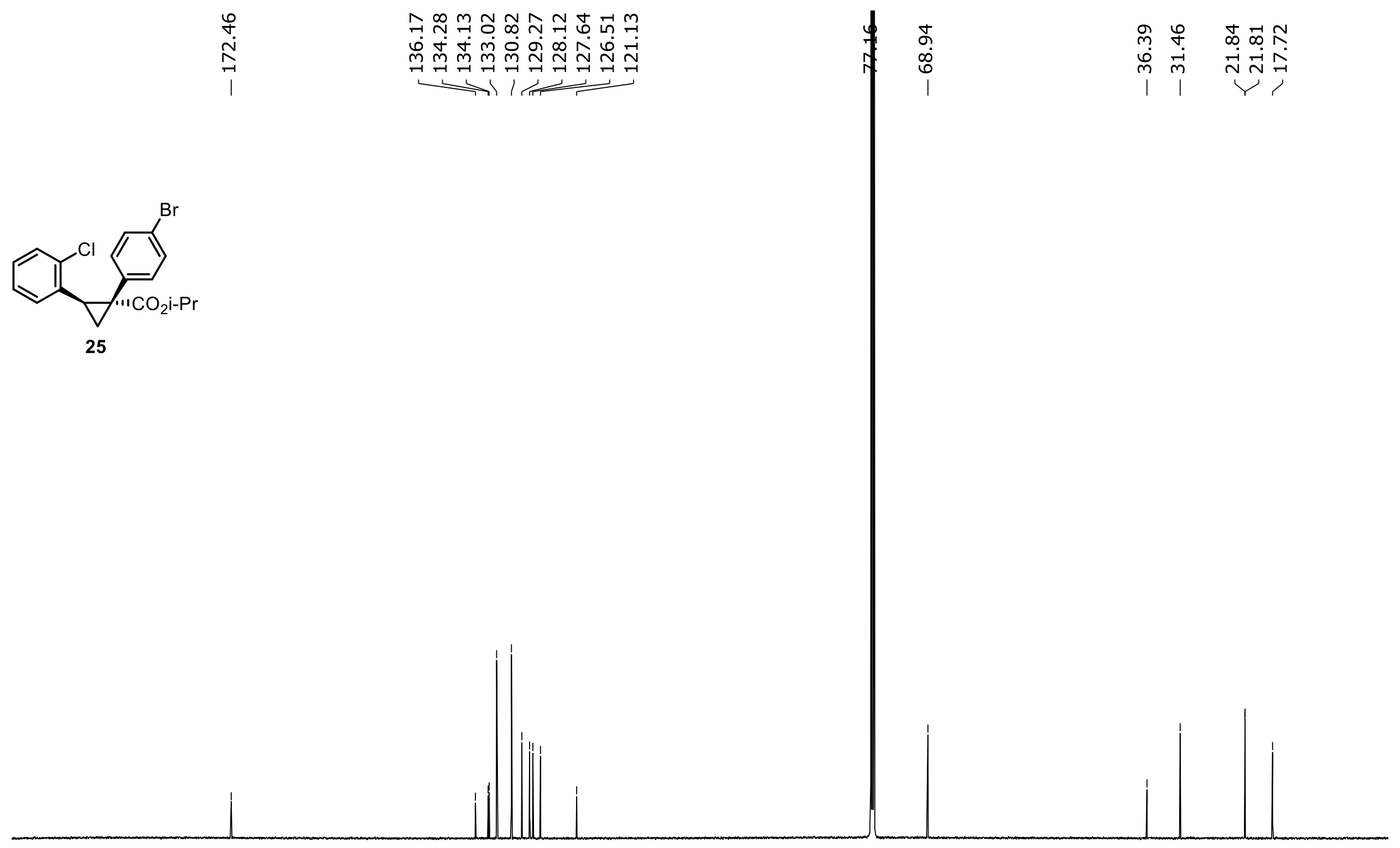

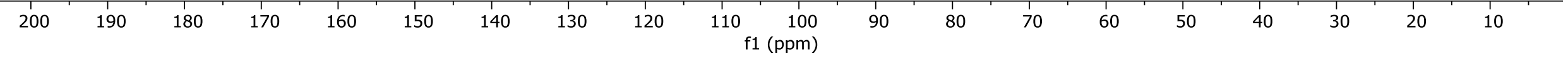

${ }^{1} \mathrm{H} \mathrm{NMR}\left(\mathrm{CDCl}_{3}, 151 \mathrm{MHz}\right)$ 
각 응ㅇㅇㅇㅇㅛ 우

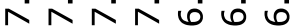

1
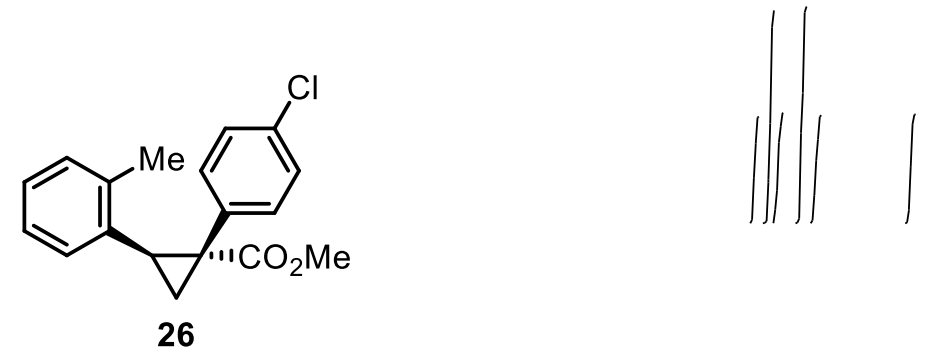

ì

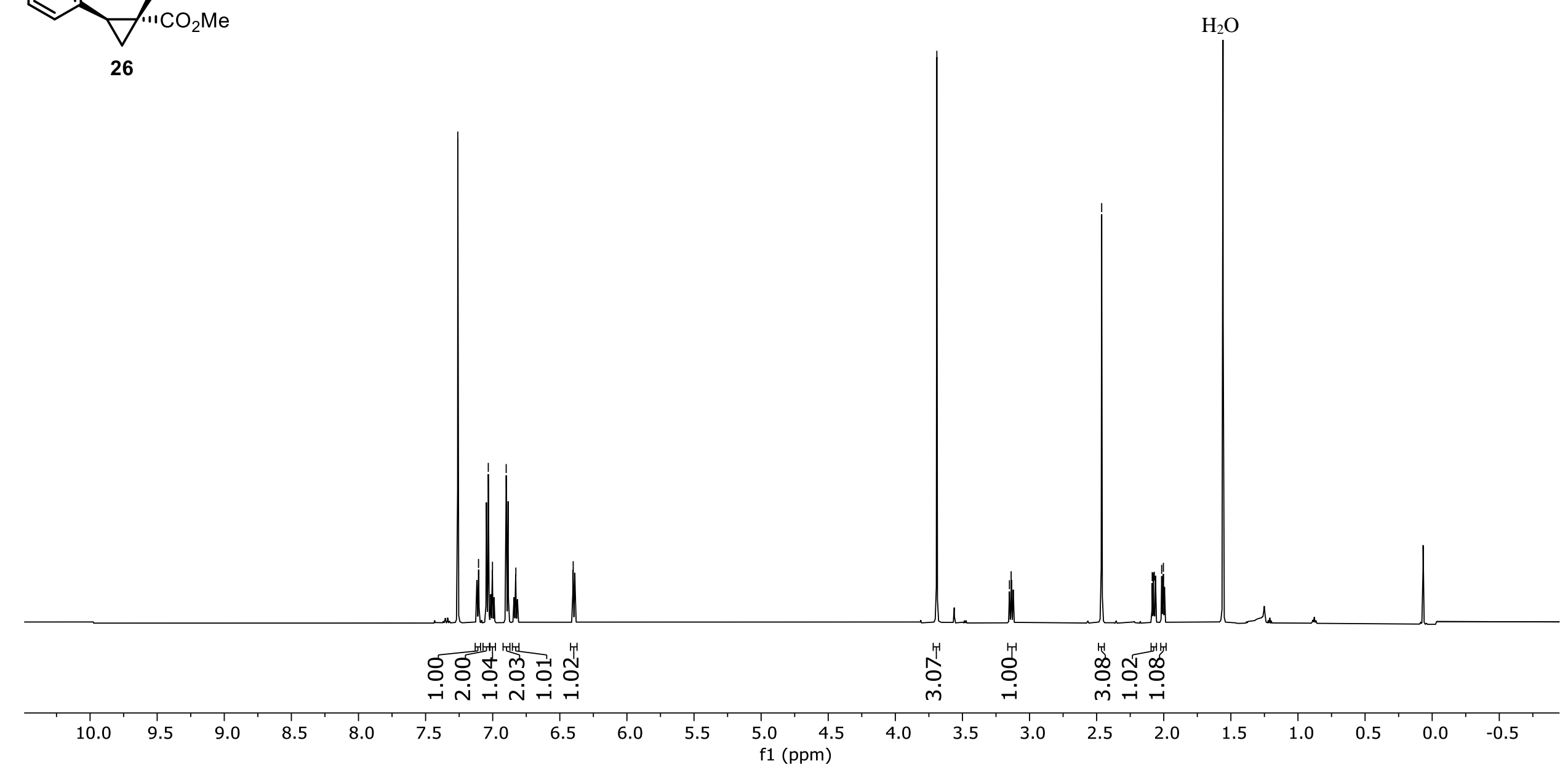

${ }^{1} \mathrm{H} \mathrm{NMR}\left(\mathrm{CDCl}_{3}, 600 \mathrm{MHz}\right)$

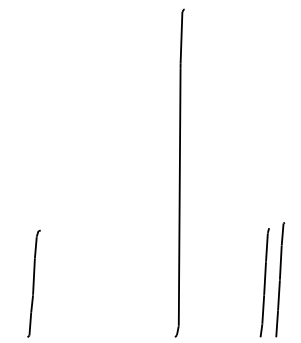




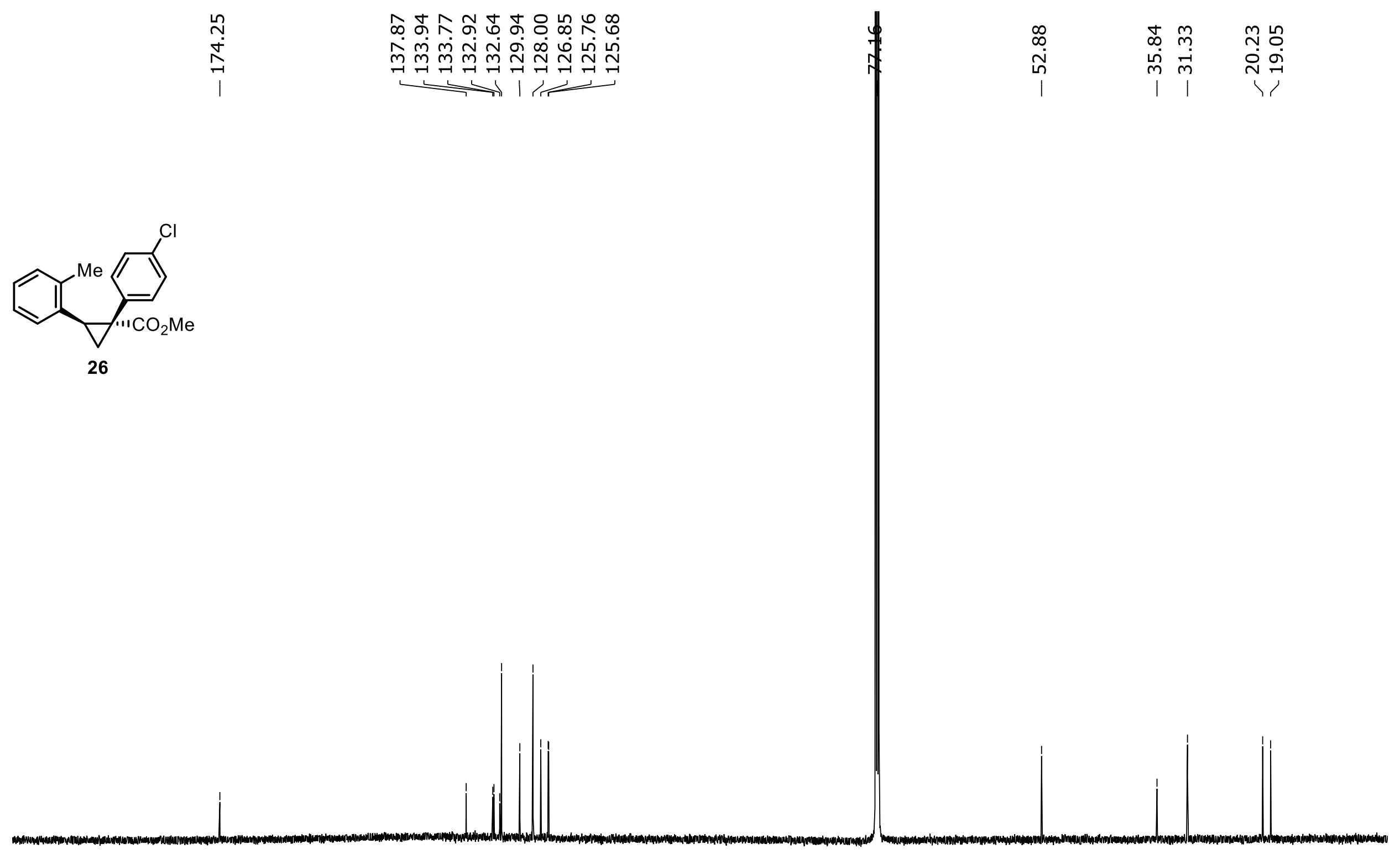

${ }^{1} \mathrm{H} \mathrm{NMR}\left(\mathrm{CDCl}_{3}, 151 \mathrm{MHz}\right)$ 


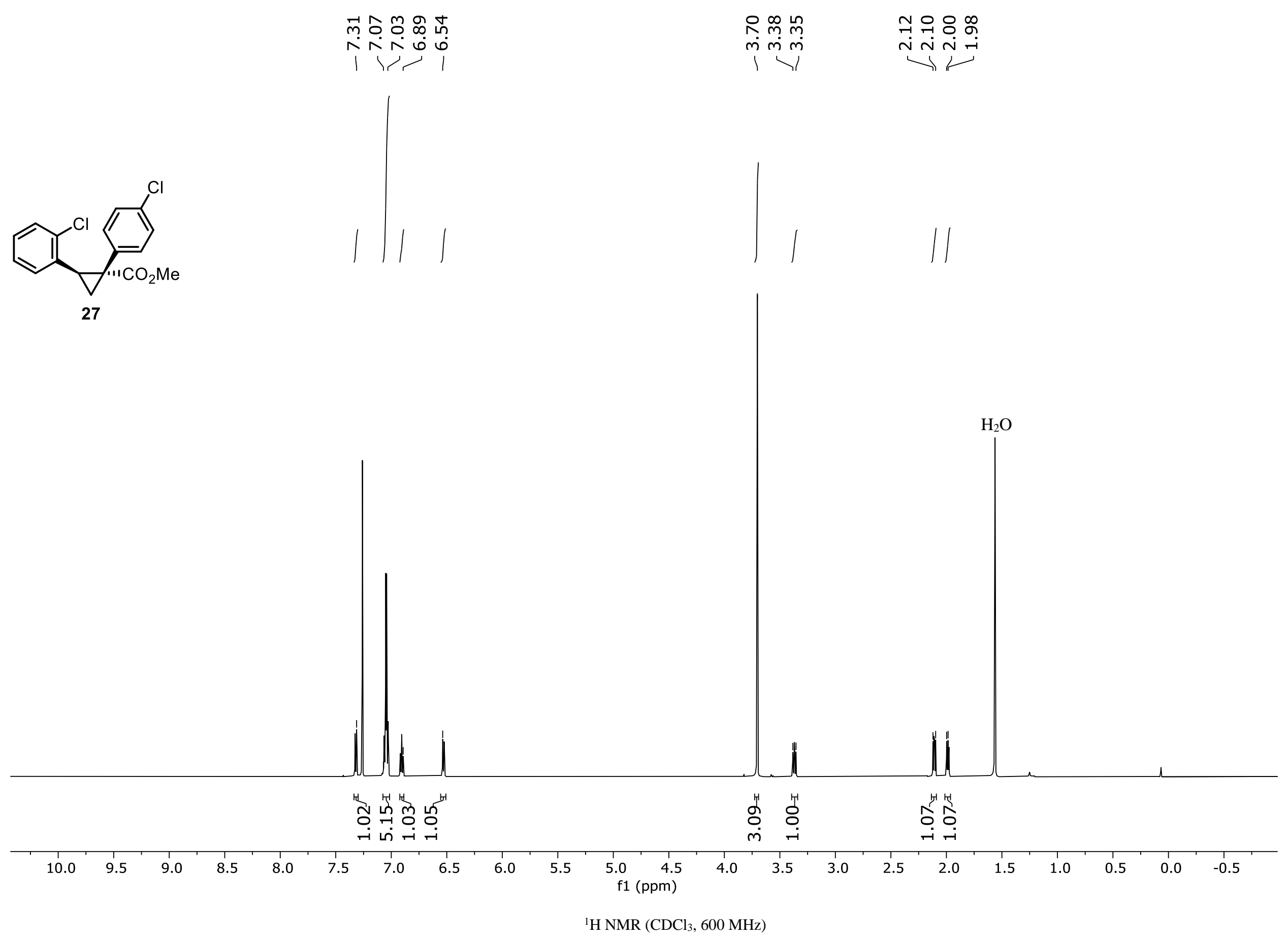



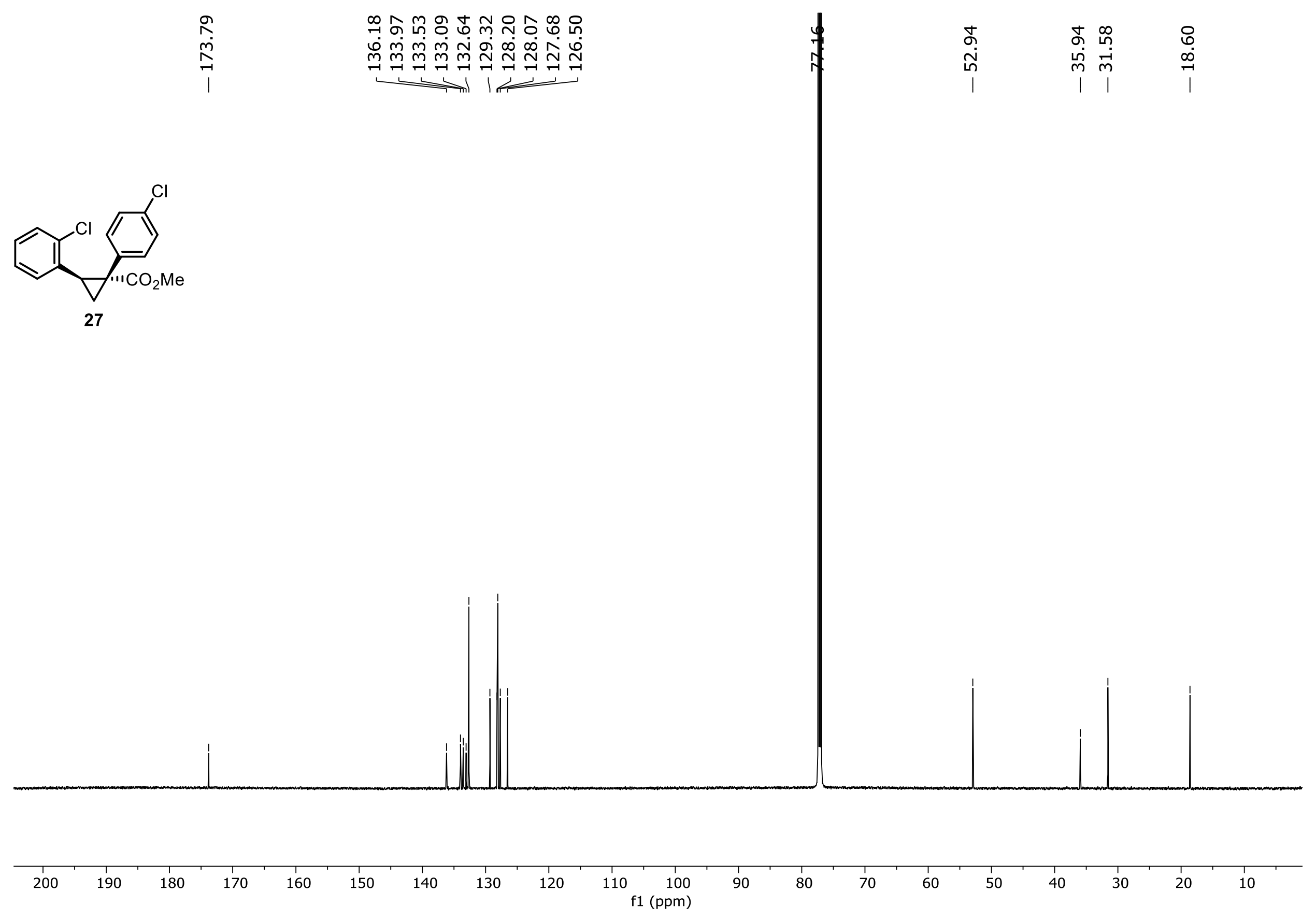

${ }^{1} \mathrm{H} \mathrm{NMR}\left(\mathrm{CDCl}_{3}, 151 \mathrm{MHz}\right)$ 


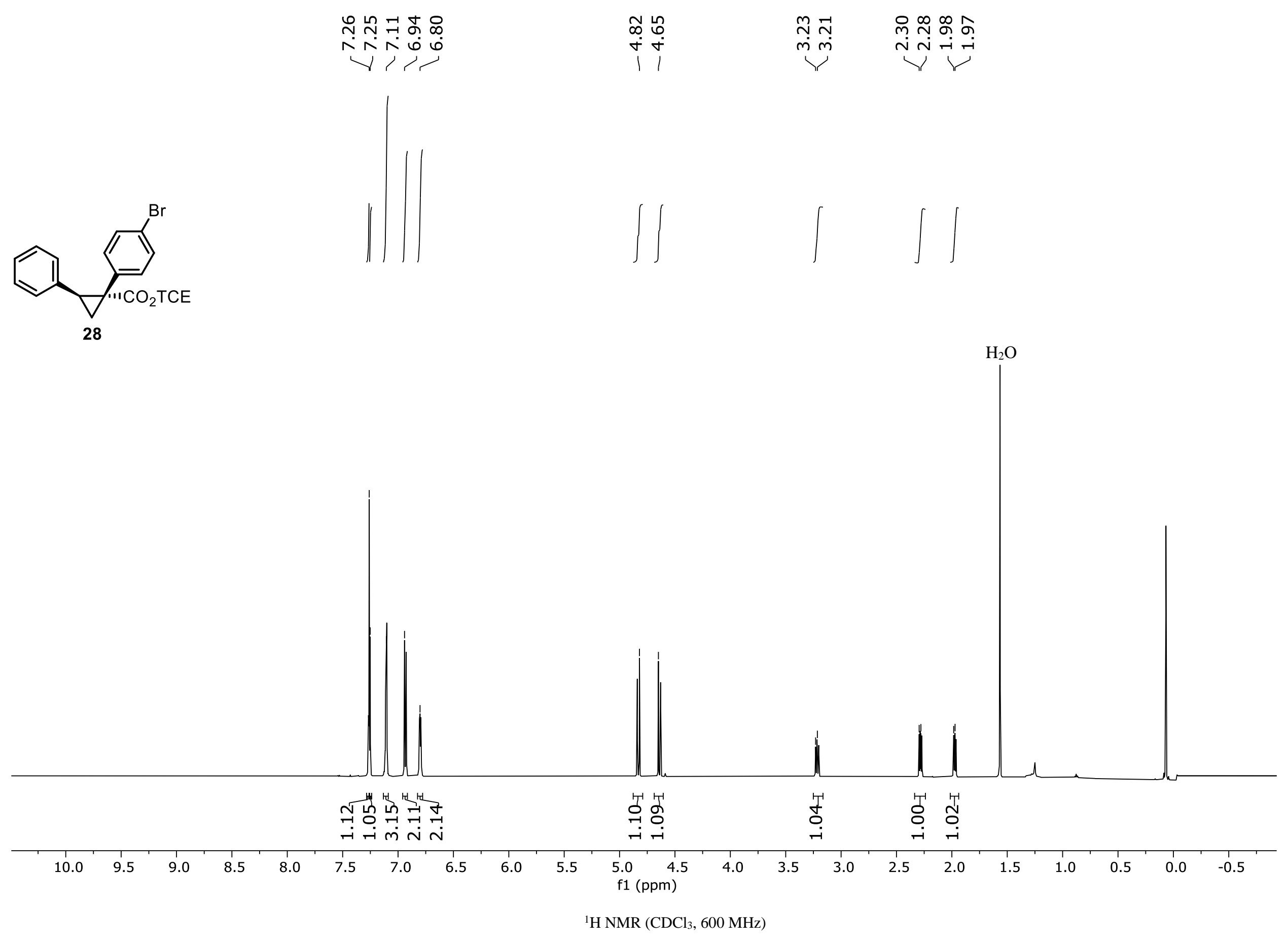




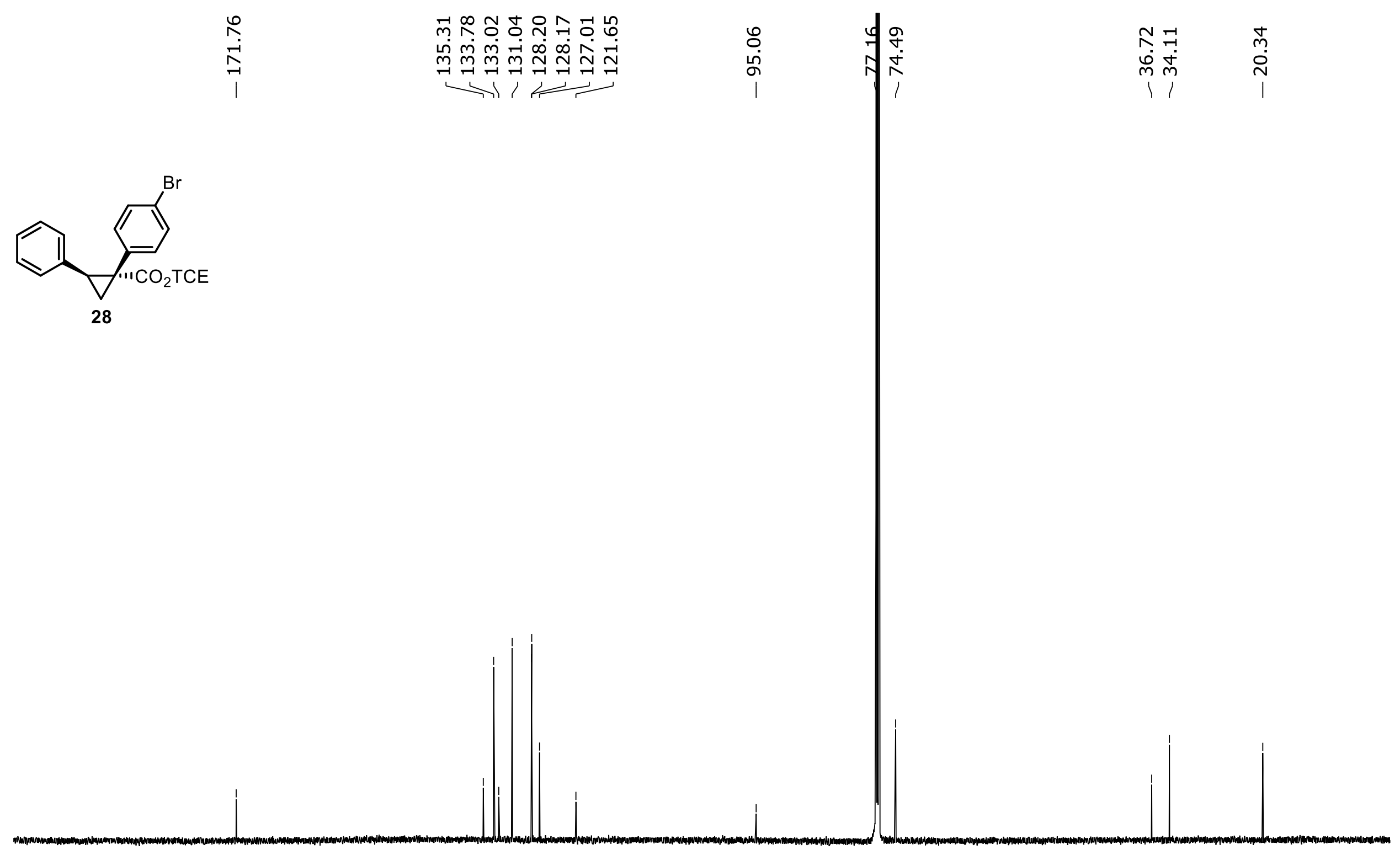

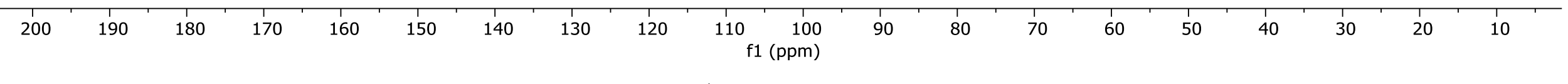

${ }^{1} \mathrm{H} \mathrm{NMR}\left(\mathrm{CDCl}_{3}, 151 \mathrm{MHz}\right)$ 

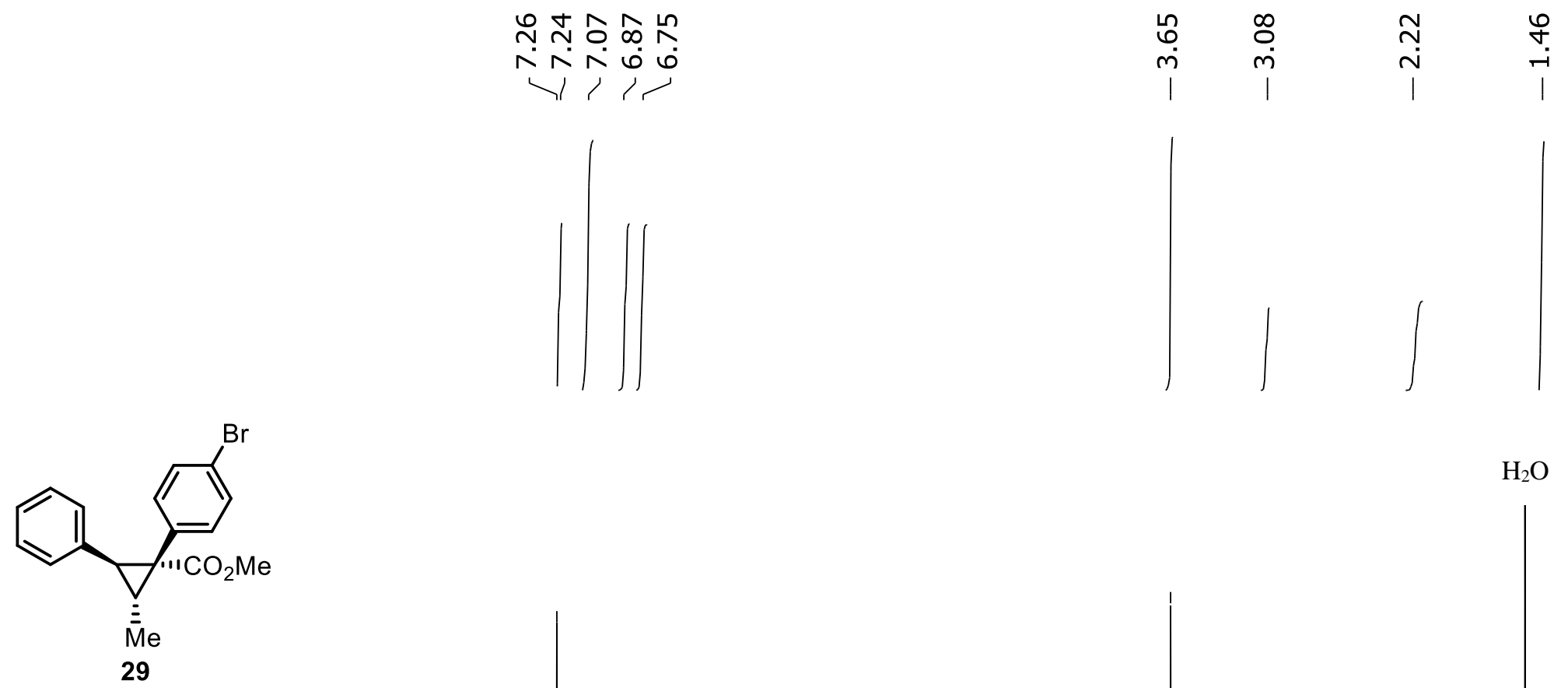

$\mathrm{H}_{2} \mathrm{O}$

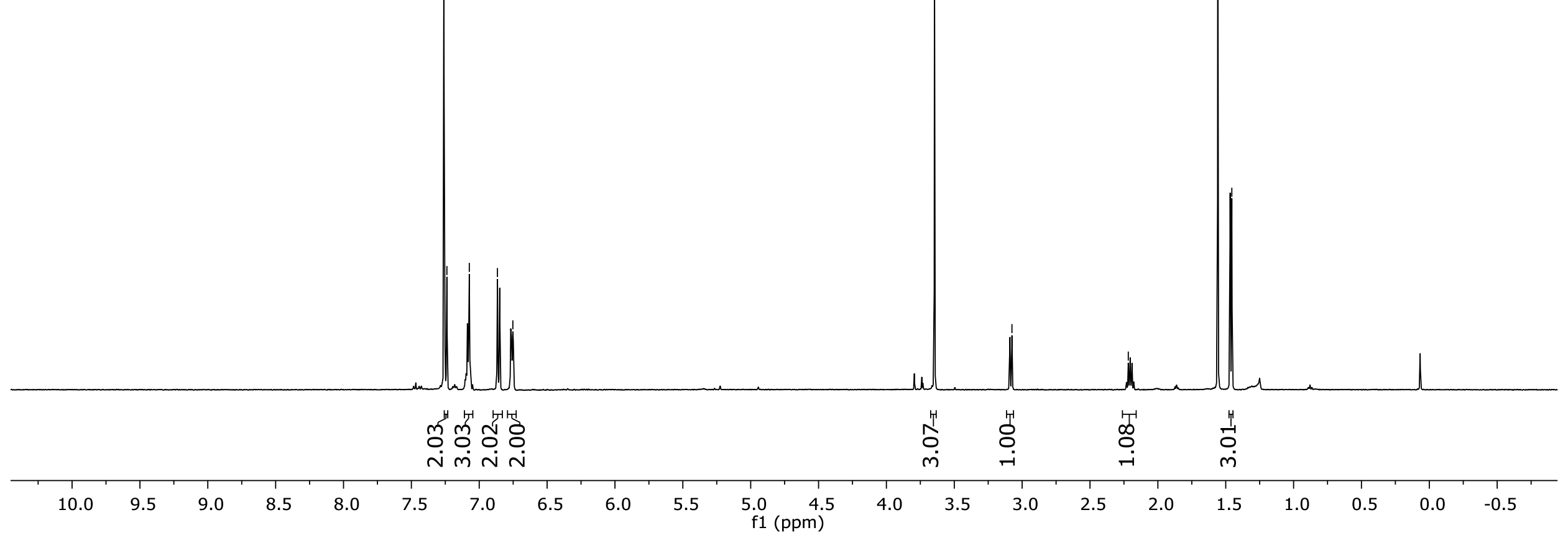

${ }^{1} \mathrm{H} \mathrm{NMR}\left(\mathrm{CDCl}_{3}, 600 \mathrm{MHz}\right)$ 

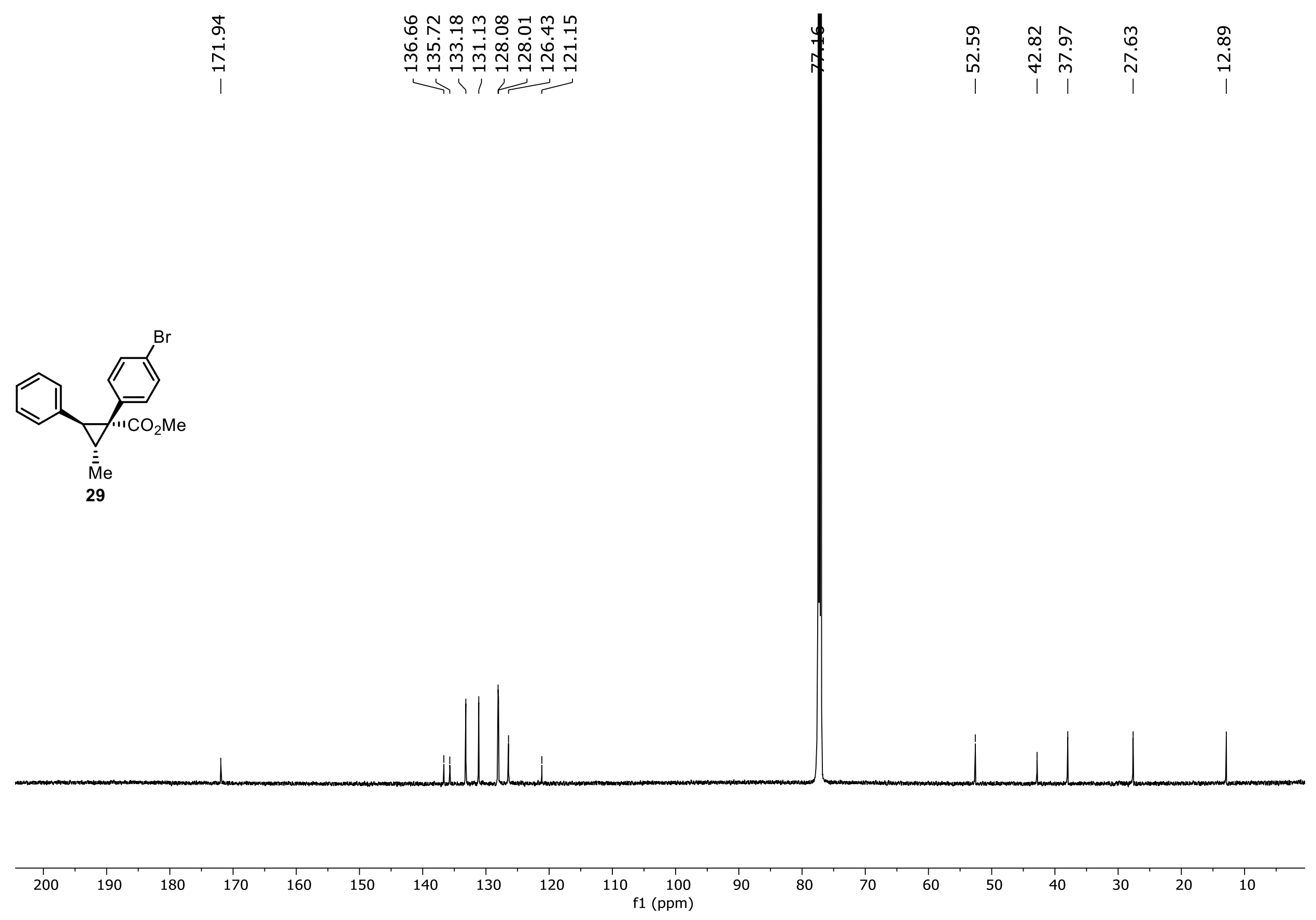

${ }^{1} \mathrm{H} \mathrm{NMR}\left(\mathrm{CDCl}_{3}, 151 \mathrm{MHz}\right)$ 


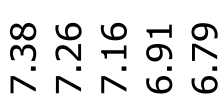

ה

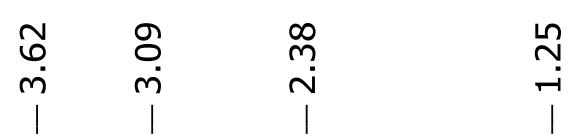
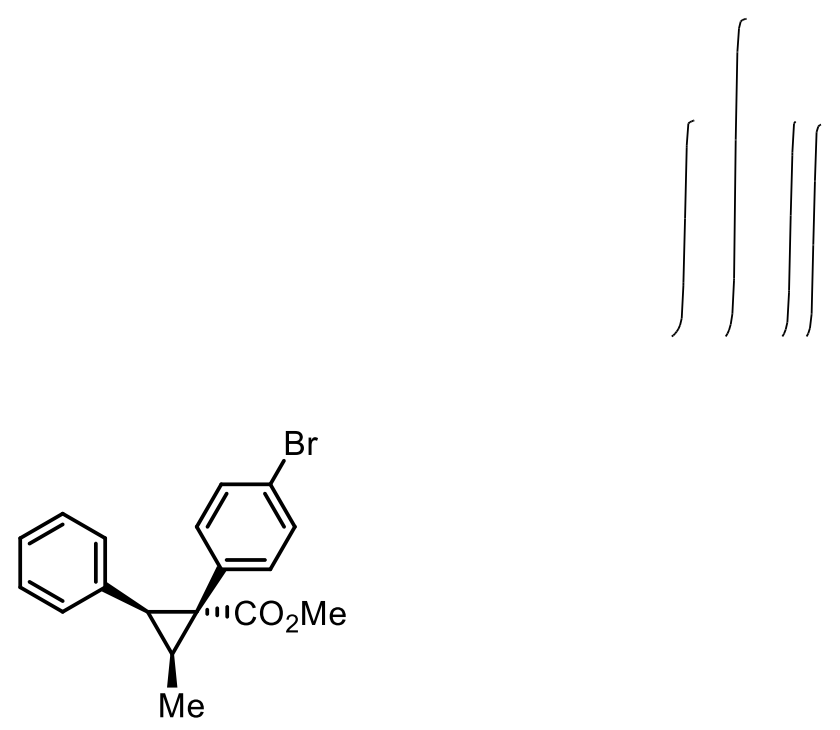

30

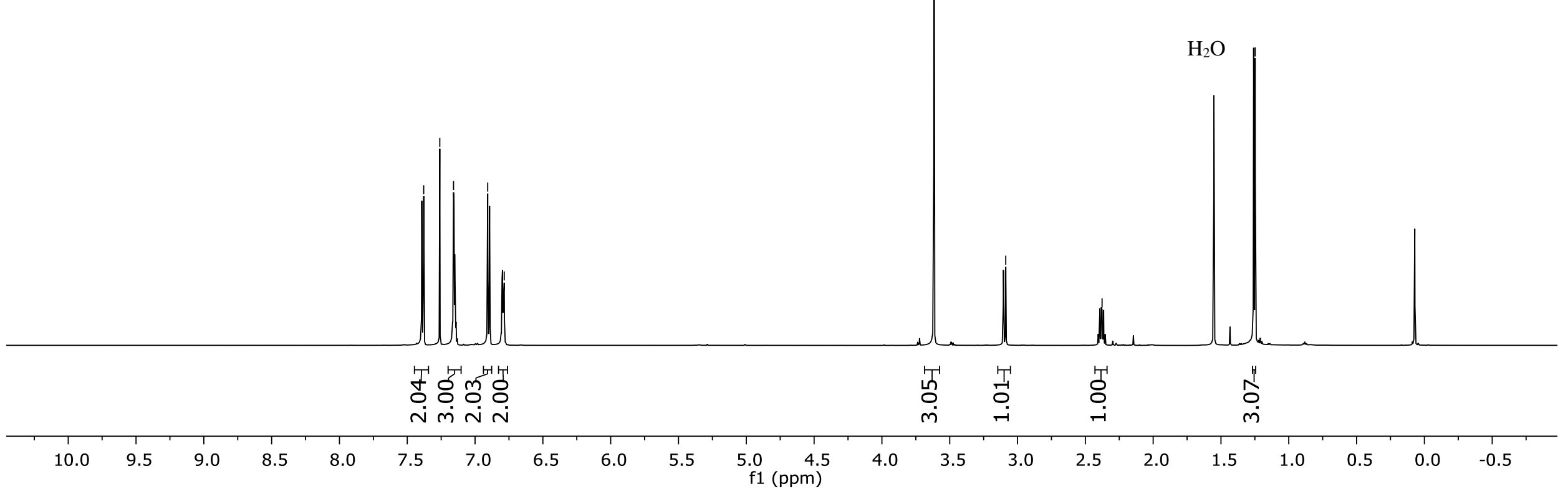

${ }^{1} \mathrm{H} \mathrm{NMR}\left(\mathrm{CDCl}_{3}, 600 \mathrm{MHz}\right)$ 


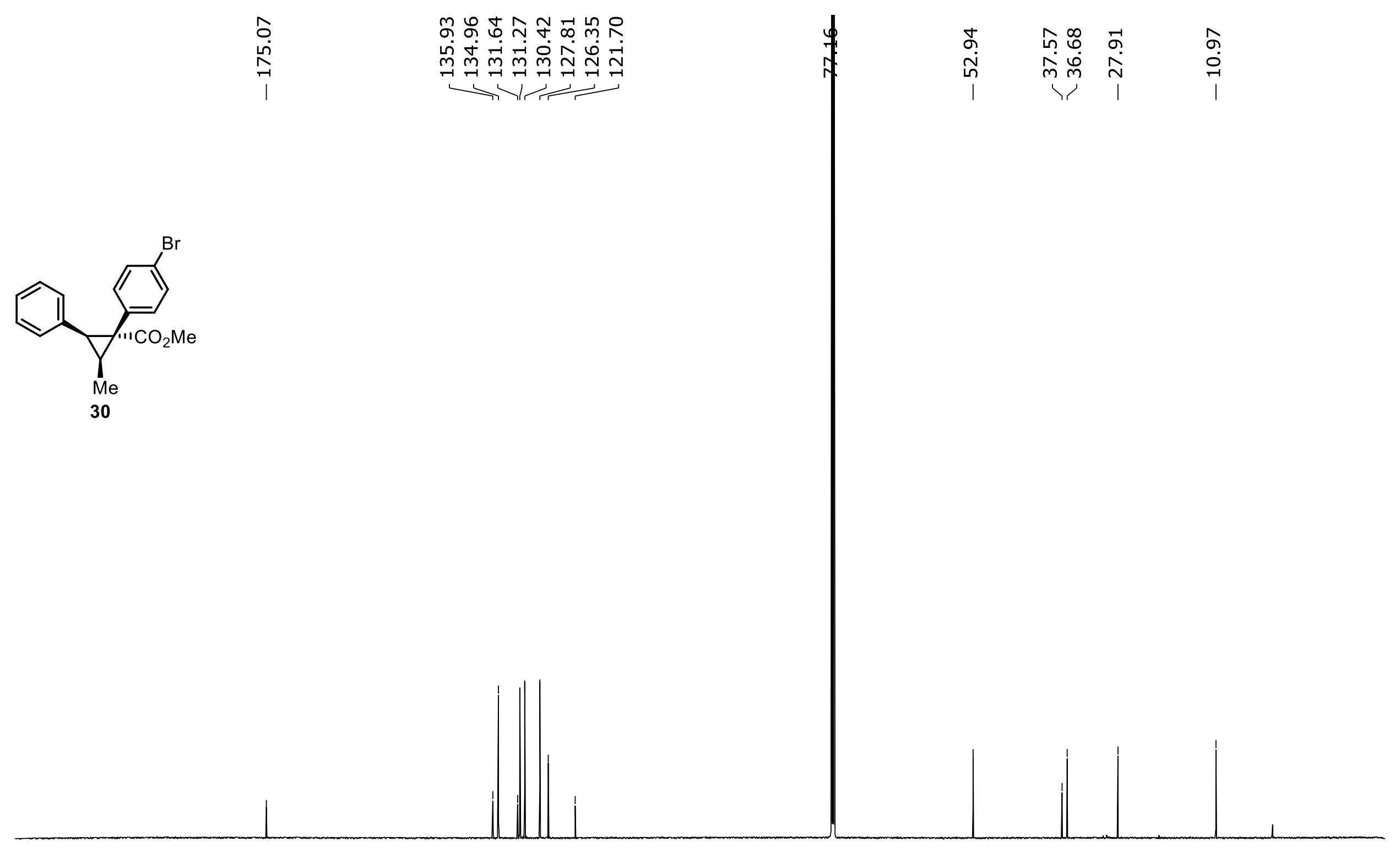

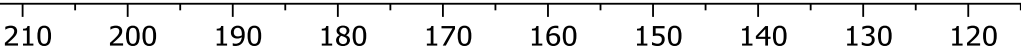
$110 \quad 100$ f1 (ppm)

${ }^{13} \mathrm{C} \mathrm{NMR}\left(\mathrm{CDCl}_{3}, 151 \mathrm{MHz}\right)$ 


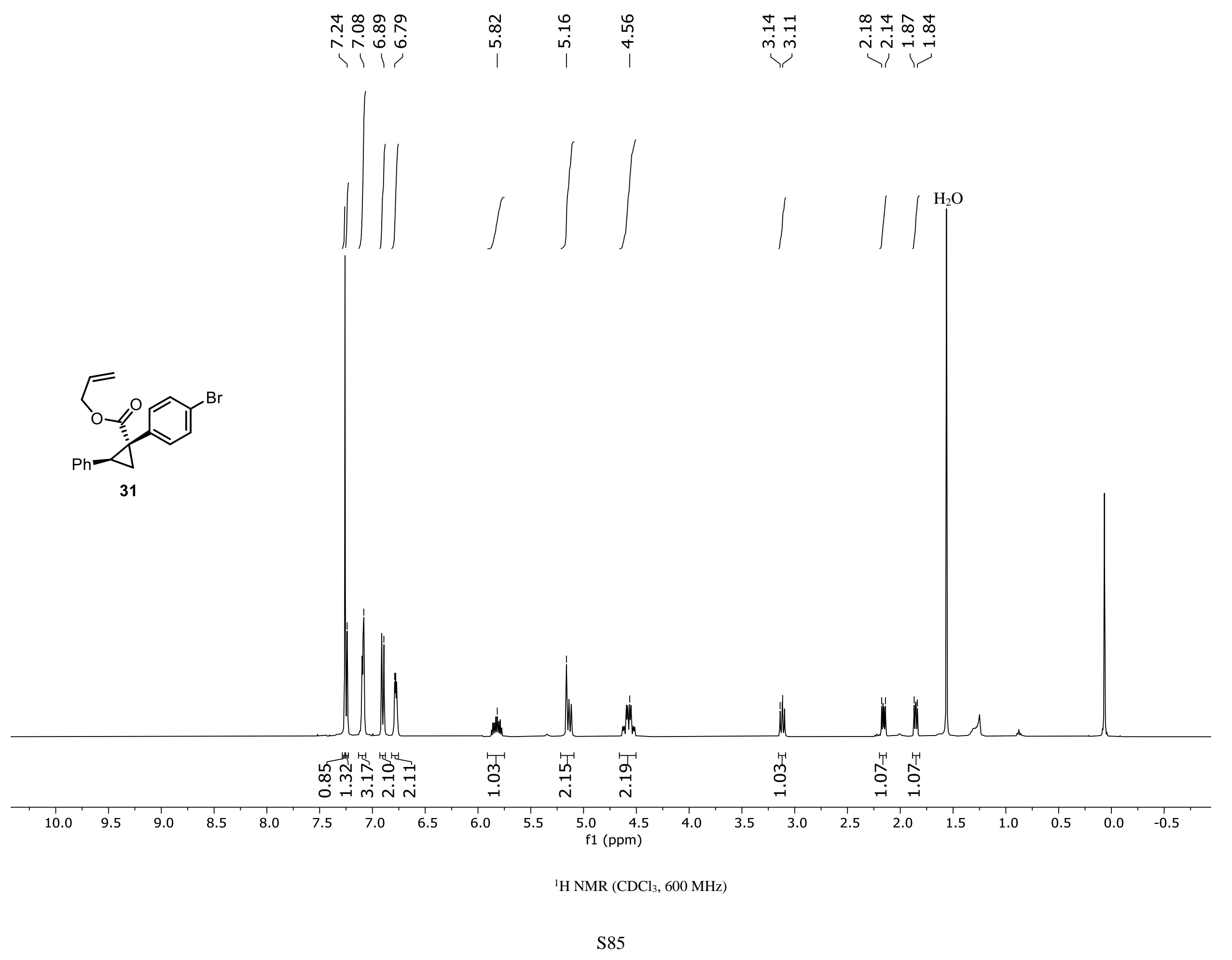


กั ำ

กิ mं
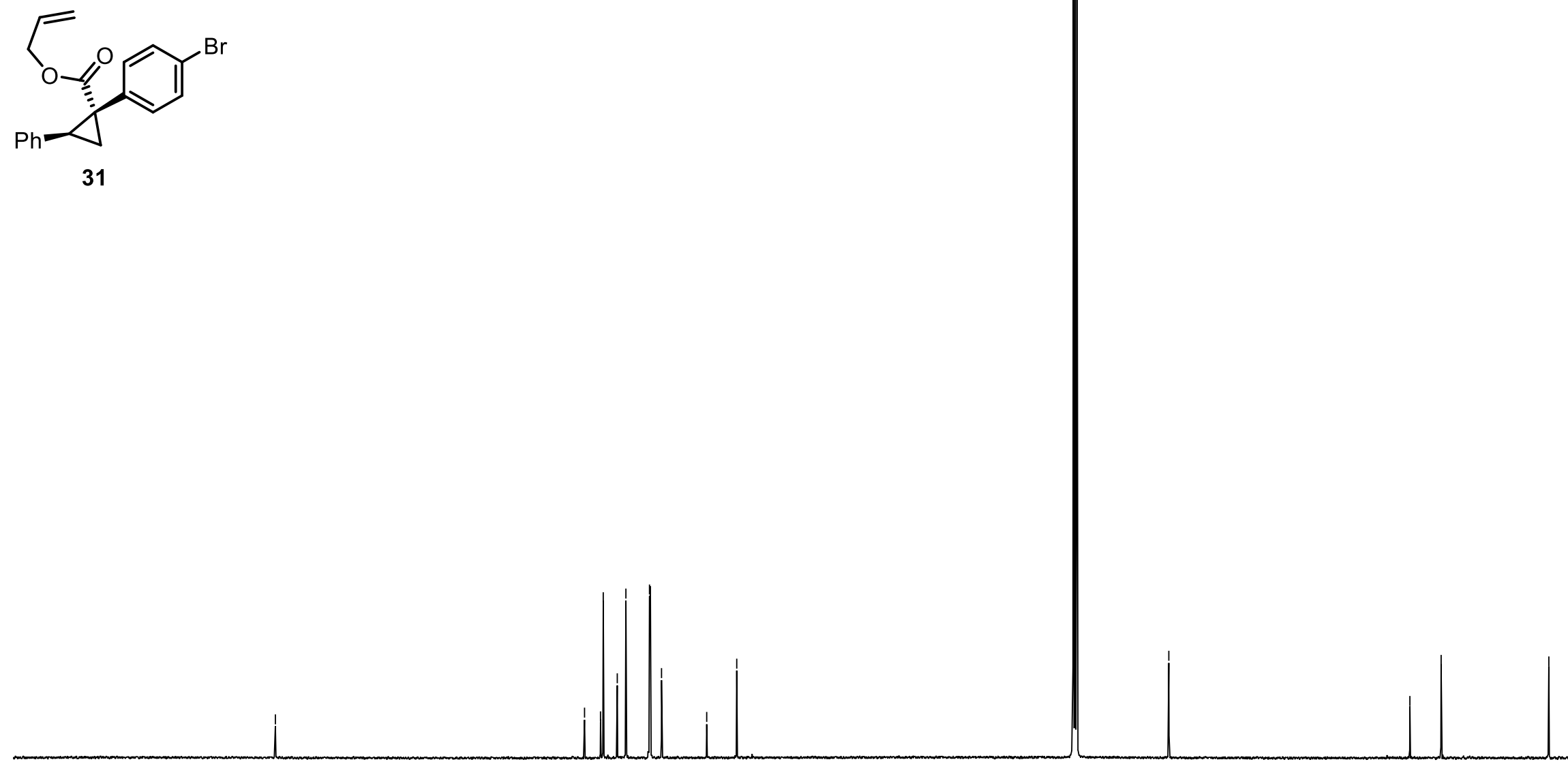

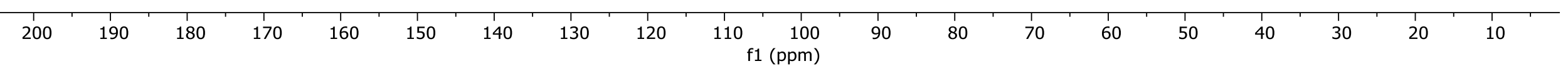

${ }^{1} \mathrm{H} \mathrm{NMR}\left(\mathrm{CDCl}_{3}, 151 \mathrm{MHz}\right)$ 


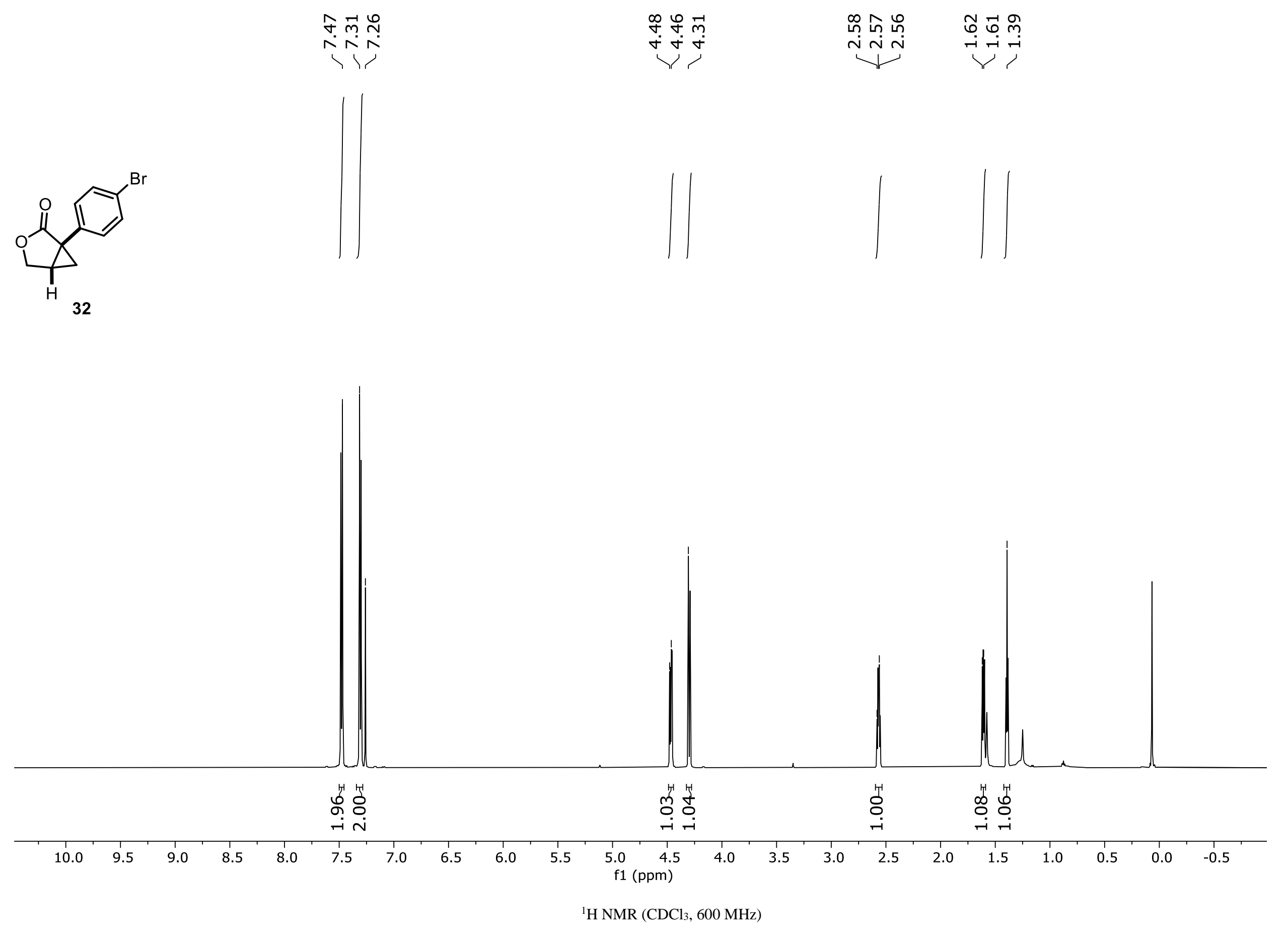




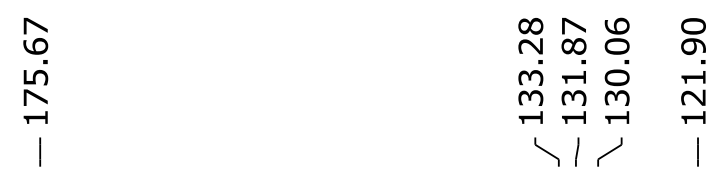

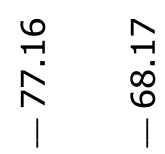

mัm

ल่ ผั่
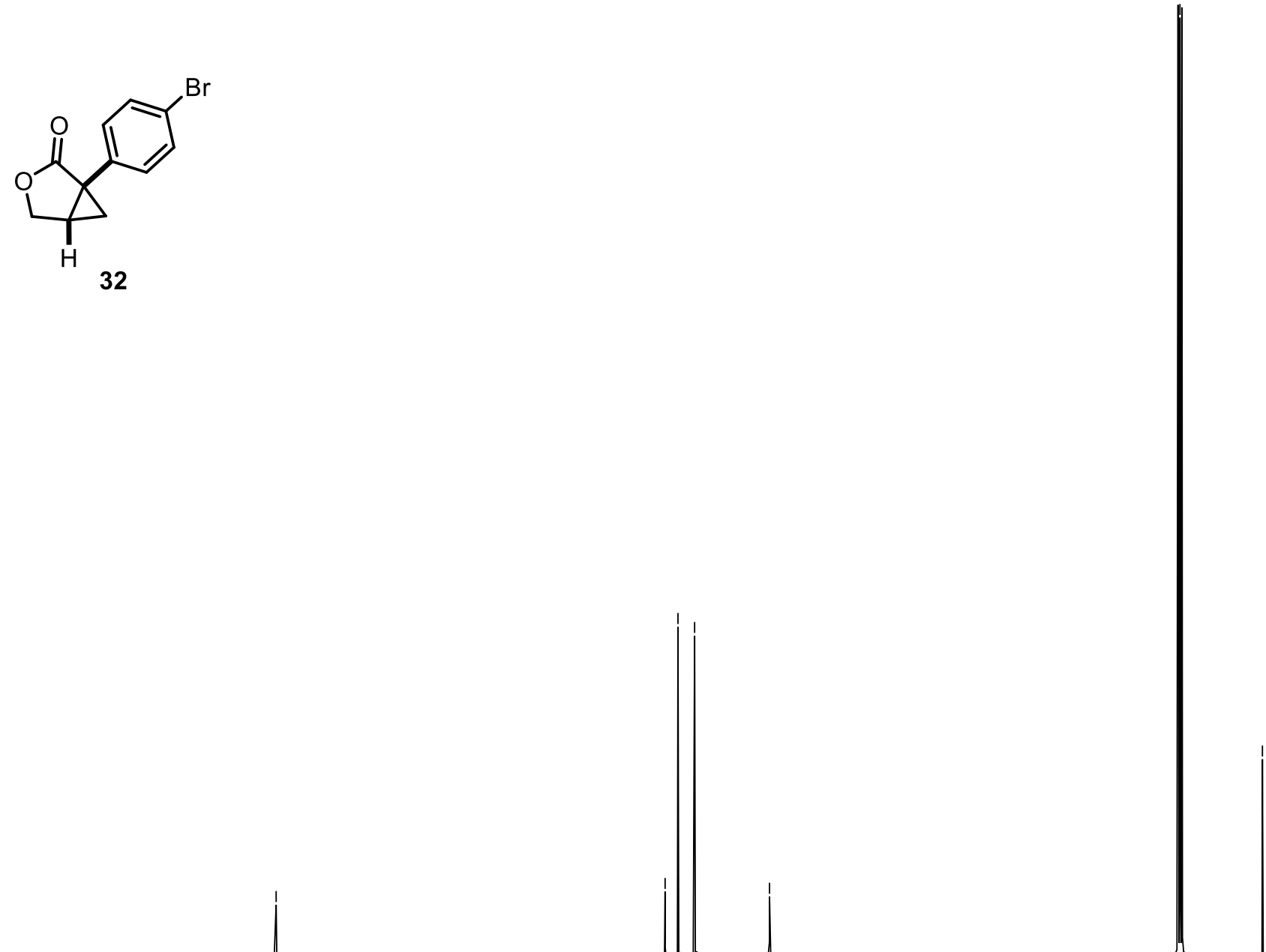

$<1<$

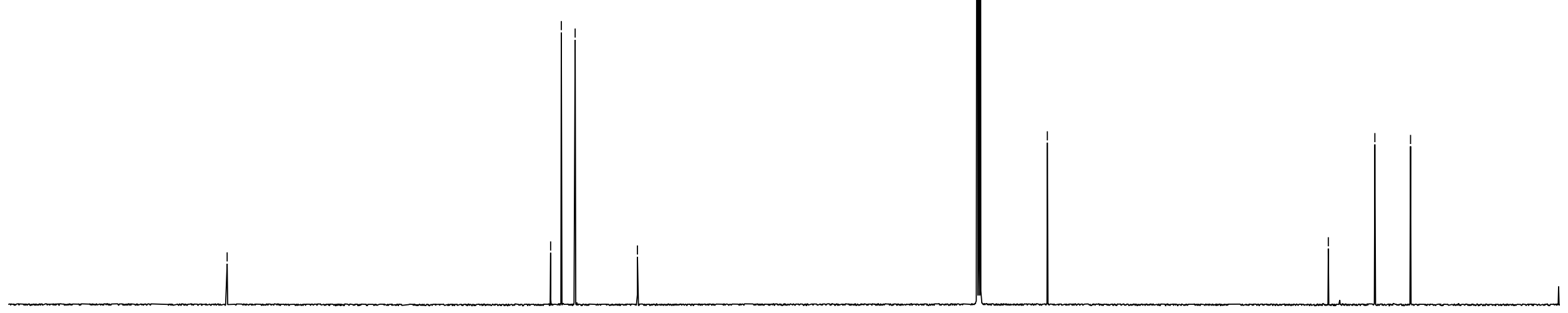

$\mathrm{H}$ NMR $\left(\mathrm{CDCl}_{3}, 151 \mathrm{MHz}\right)$ 